University of Rhode Island

DigitalCommons@URI

Open Access Dissertations

2020

\title{
A NEW PHYSICALLY MOTIVATED CLUTTER MODEL WITH APPLICATIONS TO NON-DESTRUCTIVE ULTRASONIC TESTING
}

Yazan Rawashdeh

University of Rhode Island, yazan_engineer@uri.edu

Follow this and additional works at: https://digitalcommons.uri.edu/oa_diss

\section{Recommended Citation}

Rawashdeh, Yazan, "A NEW PHYSICALLY MOTIVATED CLUTTER MODEL WITH APPLICATIONS TO NONDESTRUCTIVE ULTRASONIC TESTING" (2020). Open Access Dissertations. Paper 1183.

https://digitalcommons.uri.edu/oa_diss/1183

This Dissertation is brought to you for free and open access by DigitalCommons@URI. It has been accepted for inclusion in Open Access Dissertations by an authorized administrator of DigitalCommons@URI. For more information, please contact digitalcommons-group@uri.edu. 
A NEW PHYSICALLY MOTIVATED CLUTTER MODEL WITH APPLICATIONS TO NON-DESTRUCTIVE ULTRASONIC TESTING

BY

YAZAN RAWASHDEH

A DISSERTATION SUBMITTED IN PARTIAL FULFILLMENT OF THE REQUIREMENTS FOR THE DEGREE OF

DOCTOR OF PHILOSOPHY

IN

ELECTRICAL ENGINEERING

UNIVERSITY OF RHODE ISLAND

2020 
DOCTOR OF PHILOSOPHY DISSERTATION

$\mathrm{OF}$

YAZAN RAWASHDEH

\title{
APPROVED:
}

Dissertation Committee:

\author{
Major Professor Steven Kay \\ Haibo He \\ Araceli Medina-Bonifant \\ Nasser H. Zawia \\ DEAN OF THE GRADUATE SCHOOL
}

\section{UNIVERSITY OF RHODE ISLAND}




\begin{abstract}
The problem of detecting a defect inside the material in an ultrasonic nondestructive testing (NDT) session is addressed in this dissertation. What makes this problem a difficult one is the presence of clutter noise, which is signaldependent noise. The clutter noise in the material is caused by the microstructure of the material under test. When an ultrasonic wave travels through a coarsegrained material, the traveled pulse hits the grain boundaries, which will cause some of its energy to propagate back to the transducer and mask the echo from the defect if it exists. We tackle the problem by first establishing the statistical framework (using the hypothesis testing approach). Then, we propose a new physically motivated model for the clutter noise. We construct the physically motivated clutter model as the output of a random linear time-invariant (LTI) filter, whose impulse response can be assumed to be a realization of a Gaussian wide sense stationary (WSS) random process. Next, we determine the model mean, autocorrelation sequence (ACS), and power spectral density (PSD). The model implementation leads to the generalized matched filter (GMF) statistic and showed an advantage of more than $10 \mathrm{~dB}$ over the conventional matched filter (MF). Moreover, the model worked well on real ultrasonic data and showed robustness towards parameter misspecification. Next, we pursue the problem of the optimal signal design to be used in combination with our model. A new proof is provided for a finite data record that is lacking in the literature. We found that the optimal signal is an impulse and as a result, the signal has an impulsive ACS. Since an impulsive ACS signal is not realizable in practice a study for an alternative signal is conducted. Signals from four different categories are explored: linear frequency modulated (LFM) signal, non-linear frequency modulated (NLFM) signals, phase coded modulated (PCM) signals, and finally what we called other signals. A comparative
\end{abstract}


analysis in terms of the clutter to ambient noise ratio (CNR) versus the deflection coefficient is performed between these signals themselves and the most commonly used excitation signal in practice, which is the Gaussian amplitude modulated sinusoid (GAMS) signal. Next, we show that the LFM signal has a large advantage over the GAMS signal in terms of detectability. In addition, a comparison between the LFM signal and the GAMS signal under a deviation from the single scatterer assumption, indicating a more complex target, using simulated noise is performed. 


\section{ACKNOWLEDGMENTS}

First and foremost, I would like to express my deepest gratitude to my advisor Professor Steven Kay for his constant support and guidance throughout my PhD journey. This work could not be possible without his help. His mentorship was and still is invaluable and a student could not possibly ask for a better advisor. I appreciate his confidence in me, his patience, and his help. Being around a scholar of his caliber changed me immensely and I really appreciate it.

Next I would like to thank my thesis committee members: Professor Haibo He, Professor Araceli Medina-Bonifant, Professor Peter F. Swaszek, Professor Orlando Merino, and Professor Marco Alvarez for their help and efforts in participating in my comprehensive exam and dissertation defense.

I would like to thank Professor Frederick Vetter for his constant support and the help he provided me with throughout my study. Also, I would like to thank Professor Richard Vaccaro and Professor Ramdas Kumaresan for offering their help when needed.

I would also like to thank my parents for they are parents like no other, they gave me life, nurtured me, taught me, fought me, shouted at me, and most importantly loved me unconditionally. There are not enough words that can describe how important they are to me. I want to thank them for their constant encouragement and moral and financial support. I owe them a lot, and I hope that I can reciprocate back a bit from what they offered me, even though I know I will never be able to pay that debt. For all those times I left unsaid, thank you.

I would like to thank my sisters Diala, Dima, Rula, and Rana for their moral support and for being there when I needed them.

I would like to thank my current partner Alicia Gordon for her constant support and help. 
I left the most important person to the end I would like to thank my twin brother Yannal for his constant support in all aspects of my life. He was always and still is there for me in my ups and my downs. I could not possibly ask for a better brother and a best friend. 


\section{DEDICATION}

To my parents for they are the constant of my life and for all that I am, or hope to be, I owe to them. 


\section{PREFACE}

This dissertation is constructed in the manuscript format and consists of 2 manuscripts.

In Chapter 1, we formulate the problem statistically, then we construct a new physically motivated model for the grain scattering noise (clutter). We assume the clutter is the output of a random LTI filter, whose impulse response is considered to be a realization of a Gaussian WSS random process, when the transmitted ultrasonic pulse is at the input. Then we proceeded to derive its mean, ACS, and PSD. A comparison with the usual MF to indicate the model advantage is performed. An application to real ultrasonic data is conducted. Furthermore, we explore the effect of the choice of the model parameters. We then tested the performance under a deviation from the single scatterer assumption, for a more complex target, using simulated noise. We added a new appendix that is not a part of the paper, in the appendix we derive a probabilistically motivated model for the clutter.

Chapter 2, focuses on the optimal signal design problem to be used in combination with our model, for NDT applications. We proceed to derive analytically the optimal signal (which we found to be an impulse). Since an impulsive ACS signal is not realizable in practice, a study for an alternative signal is conducted. Signals from four different categories are explored: LFM signal, NLFM signals, PCM signals, and finally what we called other signals. A comparison analysis between these signals themselves and the most commonly used excitation signal in practice, which is the Gaussian amplitude modulated sinusoid (GAMS) signal, is implemented. In addition, a comparison between the LFM signal and the GAMS signal under a deviation from the single scatterer assumption, indicating a more complex target, using simulated noise is performed.

Finally, some possible future research works are given in Chapter 3. 


\section{TABLE OF CONTENTS}

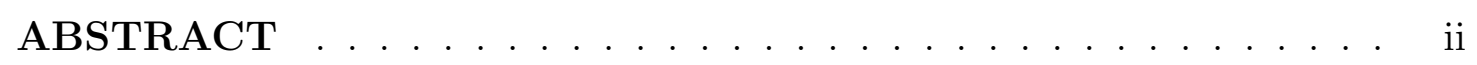

ACKNOWLEDGMENTS ................ iv

DEDICATION ....................... vi

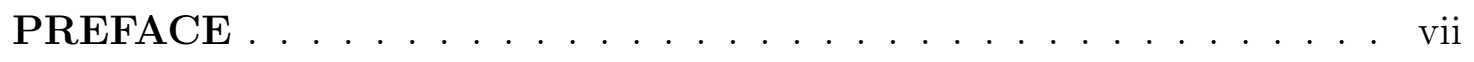

TABLE OF CONTENTS $\ldots \ldots \ldots \ldots \ldots \ldots$ viii

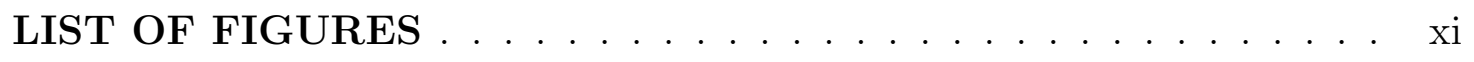

LIST OF TABLES . . . . . . . . . . . . . . . . xiii

\section{CHAPTER}

1 A New Physically Motivated Clutter Model With Applications to Non-Destructive Ultrasonic Testing . . . . . . . . . 1

Abstract .............................. 2

1.1 Introduction . . . . . . . . . . . . . . . 2

1.2 Problem Statement and Model Assumptions . . . . . . . . . 5

1.2.1 Random LTI Filter $\mathrm{h}[\mathrm{n}]$ and Clutter Noise c[n] . . . . 7

1.3 Detector Implementation and Comparison . . . . . . . . . 12

1.3.1 Matched Filter (MF) Vs Generalized Matched Filter $(\mathrm{GMF}) \ldots \ldots \ldots \ldots \ldots \ldots$

1.3.2 Practical Detector Implementation-GLRT _ . . . . . 16

1.4 Experimental results with Real Data . . . . . . . . . . 19

1.5 Parameter Sensitivity . . . . . . . . . . . . . . 22

1.6 Multiscattering for A More Complicated Target . . . . . . . . 23 


\section{Page}

1.7 Conclusion . . . . . . . . . . . . . . . . . . . . . . 26

Appendix 1A. First and Second Order Statitics and the PSD of the Physical Model . . . . . . . . . . . . . . . . . 28

Appendix 1B. Probabilistically New Model as Poisson Process . . . . 36 List of References . . . . . . . . . . . . . . . . . . . . 40

2 Optimal Signal Design For a New Physically Motivated Clutter Model With Applications to Ultrasonic Testing . . . . . . 44

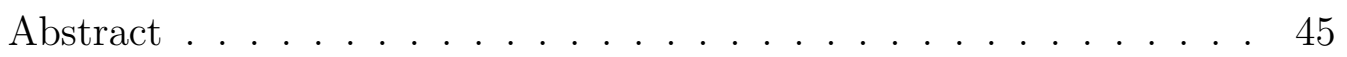

2.1 Introduction . . . . . . . . . . . . . . . 45

2.2 Problem Statement and Review of the Model . . . . . . . . . . 48

2.2.1 Model Review . . . . . . . . . . . . . . . . . . . . 50

2.3 Detector Performance . . . . . . . . . . . . . . . . . . . . 53

2.4 Maximization of The Deflection Coefficient . . . . . . . . . 55

2.5 Optimal Signal Design $\ldots \ldots \ldots \ldots$

$2.5 .1 \quad$ LFM Signal . . . . . . . . . . . . . . . . 57

$2.5 .2 \quad$ NLFM Signal . . . . . . . . . . . . . . . . . 58

2.5 .3 PCM Signals . . . . . . . . . . . . . . 60

2.5 .4 Other Signals . . . . . . . . . . . . . . . . . . 62

2.6 Comparison Analysis . . . . . . . . . . . . . . . . . . . . 66

$2.6 .1 d_{G M F}^{2}$ verses CNR and ROC curve . . . . . . . 66

2.6.2 Practical Detector-GLRT and Simulation . . . . . . . 68

2.6.3 Comparison Using Multiscattering for A More Complicated Target . . . . . . . . . . . . . . 72

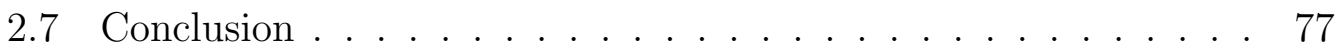


Appendix 2A. Optimal Signal Proof for Finite Data Record . . . . . . 80

List of References . . . . . . . . . . . . . . . . . . . . . 89

3 Future Work . . . . . . . . . . . . . . . . . . . 92

BIBLIOGRAPHY . . . . . . . . . . . . . . . . . . 93 


\section{LIST OF FIGURES}

Figure

Page

$1 \quad$ Modeling the received signal $x[n]: s[n]$ is the ultrasonic transmitted pulse, $h[n]$ is a random LTI filter, $g[n]$ is the impulse response of the defect, $w[n]$ is the ambient white noise, $A_{0}$ and $n_{0}$ are the scale factor and delay that correspond to the target. 6

$2 \quad$ Realization portion of $u[n], u\left[n-m_{0}\right], a[n]$, and $h[n]=a[n](u[n-$ $\left.\left.m_{0}\right]-\frac{1}{M}\right)\left(M=4\right.$ and $\left.m_{0}=2\right) \ldots \ldots . \ldots 9$

3 The signal to noise gain $S N R_{\text {Gain }}$ in dB vs clutter to ambient noise $(\mathrm{CNR})$ in $\mathrm{dB} \ldots \ldots . \ldots . \ldots 16$

4 ROC curves for both GMF (left) and MF (right) for different $A_{0} \quad 17$

$5 \quad$ Received A-scan signals $x[n] \ldots \ldots$. . . . . . . . 20

$6 \quad$ Test statistic $T_{n_{0}}(\mathbf{x})$ vs $n_{0}$ for GMF (GLRT Implementation) . . 21

$7 \quad$ Test statistic $T_{n_{0}}(\mathbf{x})$ vs $n_{0}$ for MF (GLRT Implementation) . . . 22

$8 \quad \mathrm{CNR}(\mathrm{dB}) \operatorname{Vs} d_{G M F}^{2}(d B) \ldots \ldots \ldots . \ldots . \ldots 24$

$9 \quad$ Received A-scan signals $x[n]$ with two scatterer centers . . . . . 25

10 Test statistic $T_{n_{0}}(\mathbf{x})$ vs $n_{0}$ for the Multiscatterer case . . . . 26

11 Spike process realization before applying the shift $m_{0} \ldots \ldots$. . . 29

12 Spike process realization. . . . . . . . . . . . 30

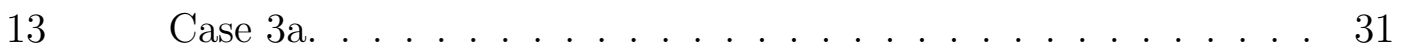

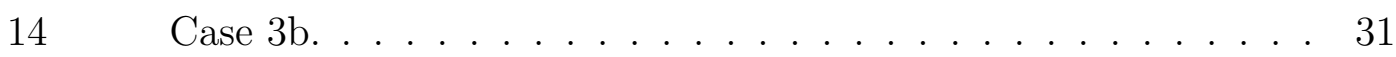

15 Linear Time Invariant Filter Impulse Response . . . . . . . . . 37

16 Linear Time Invariant System . . . . . . . . . . . . . . . 37 
17 Modeling the received signal $x[n], s[n]$ is the ultrasonic transmitted pulse, $h[n]$ is a random LTI filter, $g[n]$ is the impulse response of the defect, $w[n]$ is the ambient white noise, $A_{0}$ and $n_{0}$ are the scale factor and delay that correspond to the target. 50

18 Realization portion of $u[n], u\left[n-m_{0}\right], a[n]$, and $h[n]=a[n](u[n-$ $\left.\left.m_{0}\right]-\frac{1}{M}\right)\left(M=4\right.$ and $\left.m_{0}=2\right) \ldots \ldots . . . . . . . .52$

19 The deflection coefficient $d_{G M F}^{2}$ in $\mathrm{dB}$ vs clutter to ambient noise $(\mathrm{CNR})$ in $\mathrm{dB} \ldots \ldots . \ldots . \ldots . . \ldots 67$

20 The deflection coefficient $d_{G M F}^{2}$ in $\mathrm{dB}$ vs clutter to ambient noise $(\mathrm{CNR})$ in $\mathrm{dB} \ldots \ldots . \ldots . \ldots . . \ldots 68$

21 ROC curves for both LFM signal (Left) and the GAMS signal (Right) for different energy levels (Amplitude) . . . . . . . . 69

22 Received A-scan signals $x[n]$ using the LFM signal . . . . . . . 71

23 Received A-scan signals $x[n]$ using the GAMS signal . . . . . . 72

24 Test statistic $T_{n_{0}}(\mathbf{x})$ vs $n_{0}$ for GMF using LFM signal (GLRT Implementation) . . . . . . . . . . . . . . . 73

25 Test statistic $T_{n_{0}}(\mathbf{x})$ vs $n_{0}$ for GMF using GAMS signal(GLRT Implementation) . . . . . . . . . . . . . 74

26 Received A-scan signals $x[n]$ using the LFM signal . . . . . . . 75

27 Received A-scan signals $x[n]$ using the GAMS signal . . . . . . 76

28 Test statistic $T_{n_{0}}(\mathbf{x})$ vs $n_{0}$ using LFM signal (GLRT Implemen-

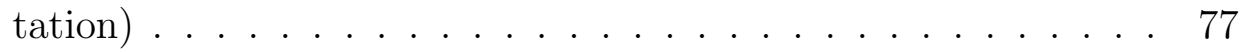

29 Test statistic $T_{n_{0}}(\mathbf{x})$ vs $n_{0}$ using GAMS signal (GLRT Imple-

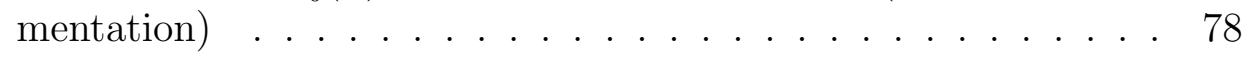




\section{LIST OF TABLES}

Table

Page

1 Test Statistics $T_{n_{0}}(\mathbf{x})$ of all four real data waveforms for different combinations of $M$ and $\sigma_{A}^{2} \ldots \ldots . \ldots 23$

2 Defect signal energy levels verses probability of detection $P_{D}$ for both LFM and GAMS signals . . . . . . . . . . . . 71

3 Defect signal energy levels verses probability of detection $P_{D}$ for both LFM and GAMS signals for A More Complicated Target . 76 


\title{
CHAPTER 1
}

\section{A New Physically Motivated Clutter Model With Applications to Non-Destructive Ultrasonic Testing}

\author{
by \\ Yazan Rawashdeh and Steven Kay \\ Dept. of Electrical, Computer and Biomedical Engineering \\ University of Rhode Island, Kingston, RI, USA
}

Published in IEEE Transactions on Ultrasonics, Ferroelectrics, and Frequency Control, 2020. 


\begin{abstract}
In this paper, we construct a new physically motivated model for the grain scattering noise (clutter). We assume the clutter is the output of a random linear time-invariant (LTI) filter, whose impulse response is considered to be a realization of a Gaussian wide sense stationary (WSS) random process, when the transmitted ultrasonic pulse is at the input. In an ultrasonic non-destructive testing (NDT) session, the clutter noise, which is signal-dependent and caused by the microstructure of the material, presents a substantial challenge in identifying defects in the material under testing. The model is used to aid in the detection of a defect in the material. The model incorporates, explicitly, many important physical characteristics of the generated clutter: the average grain size, the random shape and orientation of the grains, and emphasizes the single scatterer assumption (Rayleigh region). The statistical properties of the model are formulated and derived. A comparison with the usual matched filter to indicate the model advantage is performed. An application to real ultrasonic data is conducted with excellent results. Furthermore, we explored the effect of the choice of the model parameters, and the model shows robustness towards parameter misspecification. We then tested the performance under a deviation from the single scatterer assumption, for a more complex target, using simulated noise and obtained promising results.
\end{abstract}

\title{
$1.1 \quad$ Introduction
}

The problem of detecting a target in signal-dependent noise has been researched extensively throughout the years. Signal-dependent noise is referred to as clutter in radar, backscattering noise in ultrasonic non-destructive testing (NDT) of materials, and reverberation in sonar. However, the term clutter has been used interchangeably with backscattering noise, and we will follow this convention here. In an ultrasonic NDT session, a piezoelectric transducer fires a short-duration pulse 
of ultrasound in a narrow beam into the material and any echoes coming back are received with the same transducer [1]. The goal of examining the collected data can be categorized into two subcategories, one is detection (detecting if a defect exists in the scanned material), and the other is classification (classify the type of defect, if it exists). A non-destructive test is one in which there is no impairment of the properties and performance in future use of the object under examination $[2]$.

In this work, we construct a physically motivated clutter model as the output of a random linear time-invariant (LTI) filter, whose impulse response can be assumed to be a realization of a Gaussian wide sense stationary (WSS) random process. The model is used to aid in the detection phase of examining the data. Modeling the clutter as the output of a random LTI filter, whose impulse response is assumed to be a WSS random process has been used before in radar problems [3], [4], [5]. The term physically motivated indicates that we took into account some of the important physical characteristics of the grains in the material that generated the clutter, such as the average grain size and the random orientation and shape of the grains. In addition, we emphasize the single scatterer assumption (Rayleigh region), in contrast to multi-scattering, which results when the wavelength of the test signal becomes very close to the size of the general diameter of the grains [6]. Also, we will assume that the material under test is homogenous (uniform grain size throughout the materials) and isotropic (the material has identical property values in all directions). Excellent results from applying the model on real ultrasonic data were obtained, and the model exhibits robustness towards parameter misspecification.

The clutter noise in NDT is caused by the microstructure of the material under test. When an ultrasonic wave travels through a coarse-grained material, 
the traveled pulse hits the grain boundaries, which will cause some of its energy to propagate back to the transducer. Single grain size in such a material is usually very small, and the reflected amount of energy is minimal from each grain. However, the large number of grains in a scanned volume of the material, taking into account the randomness of their size, orientation, and shape, will result in an in-phase addition and a more considerable amount of energy to be reflected back to the transducer. Even though the flaw size is usually much larger than an individual grain, in many cases, these reflected energies mask the echoed energy from a flaw located in the same path of the scanned volume. In general, in coarsegrained materials, acoustic wave propagation is a function of the microstructure, frequency, and wave mode [7]. An elaborate discussion for the ultrasonic NDT, acoustic wave propagation in materials, and clutter noise characteristics can be found in [1], [2], [8], [9] and the references within.

Many algorithms have been proposed to tackle the above problem in either the frequency domain, time domain, or both. Some of the proposed methods are simple filtering, Wiener filtering, split spectrum, wavelet filtering, spectrum equalization, empirical mode decompositions, even the use of the neural network. Many of these methods are considered nonparametric such as split spectrum [10], [11] and some are considered parametric such as [12], [13]. In the parametric approach, a model that depends on some parameters is chosen, and these parameters are estimated from the collected data (our approach will be of this type). On the other hand, the nonparametric approach does not make such an assumption and uses the collected data directly. Proposed models for the clutter noise in materials range in simplicity and complexity, depending on their applications. Many models have been provided for simulation purposes with no statistical framework [10], [14], [15], [16]. Some other models include a statistical framework such as [17], where the 
model is assumed to be delayed, distorted, and scaled versions of the transmitted pulse. However, the problem was tackled in the frequency domain and without any reference to the detection problem. A similar model to the previous one can be found in [12], where the grain noise is modeled as a Gauss-Markov random field and uses the likelihood ratio test as a detection tool, and has shown good results in detecting flaws in stainless steel material based on ultrasound images. In [18], their work focused on the amplitude variations statistics and not the detection problem. Many models assume that the delays of the reflected signals are uniformly distributed over the scanned volume [10], [12], [17], [18], to account for the grain's random orientation, shape, and size. However, such an assumption raises many improbable situations that are not physically possible in the material, such as having three or more scatterer centers less than an average grain size apart when the assumption to ignore the multi-scatterer is assumed. In contrast, in our model, we tried to emphasize the single scatterer assumption by considering a single scatterer center per average grain size, and later we explored the multi-scatterer assumption for a more complicated target.

In this study, we first state the detection problem and provide the model assumptions in section 1.2, followed by a performance comparison with the usual matched filter and the detector implementation in section 1.3. Next, in section 1.4 we apply the model on real ultrasonic data and discuss the outcomes. In section 1.5 we explore the model parameters sensitivity and in section 1.6 we discuss the multiscatterer centers for a more complicated target. Finally, conclusions are given in section 1.7 .

\subsection{Problem Statement and Model Assumptions}

The model for the received signal shown in Fig. 1.

$$
\begin{array}{ll}
\mathcal{H}_{0}: & x[n]=w[n]+c[n] \\
\mathcal{H}_{1}: & x[n]=A_{0} s\left[n-n_{0}\right]+c[n]+w[n]
\end{array}
$$




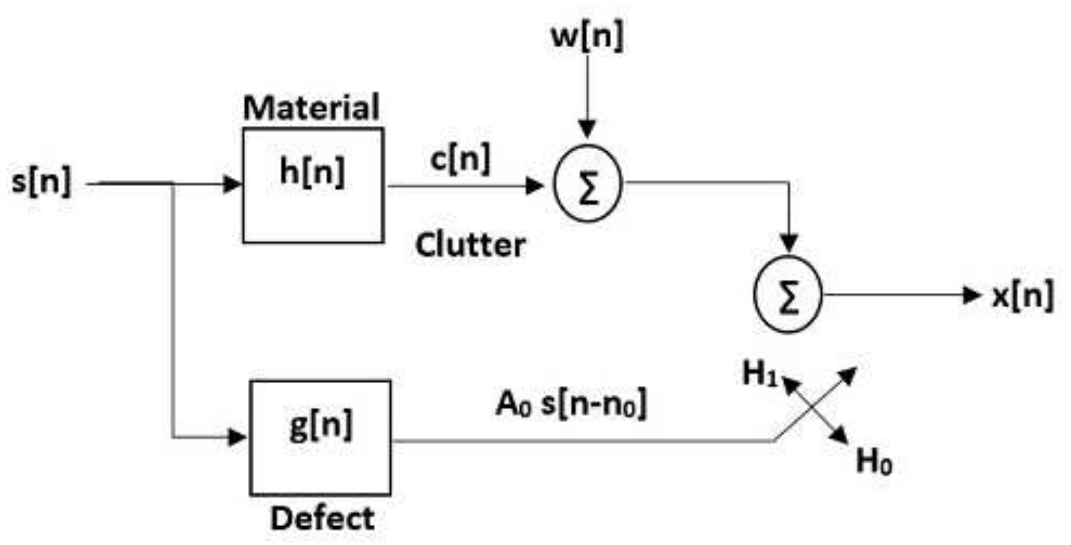

Figure 1: Modeling the received signal $x[n]: s[n]$ is the ultrasonic transmitted pulse, $h[n]$ is a random LTI filter, $g[n]$ is the impulse response of the defect, $w[n]$ is the ambient white noise, $A_{0}$ and $n_{0}$ are the scale factor and delay that correspond to the target.

To formulate the problem in a statistical framework we assume that the received signal is $x[n]=c[n]+w[n]$ under hypothesis $\mathcal{H}_{0}$, where $c[n]$ denotes the clutter noise, and $w[n]$ denotes the ambient white noise. Under the hypothesis $\mathcal{H}_{1}$, we get $x[n]=A_{0} s\left[n-n_{0}\right]+c[n]+w[n]$, where the defect impulse response $g[n]$ is modeled as a point target (only a shift and scaling of the transmitted signal $s[n])$. The amplitude $A_{0}$ and delay $n_{0}$ represent the scale factor and delay that correspond to the target geometry and location respectively. The transmitted ultrasonic pulse $s[n]$ is specified by the type of transducer excitation that is used for testing. The optimal signal design problem is beyond the scope of this paper, and will be addressed in a future work. We then modeled the clutter $c[n]$ as the output of a random LTI filter with the impulse response $h[n]$, when the input is the transmitted ultrasonic pulse $s[n]$.

As shown in Fig. 1 there are two types of noise that will affect our received signal. First, the ambient white noise $w[n]$ that is introduced in the instrumen- 
tation (circuitry, A/D conversion, temperature variations) and other artifacts [7], and is assumed to be an independent identically distributed (IID) white Gaussian noise process with a probability density function $(\mathrm{PDF}) \mathbf{w} \sim \mathcal{N}\left(\mathbf{0}, \sigma_{w}^{2} \mathbf{I}\right)$, where the boldface $\mathbf{w}$ indicates the vector of ambient white noise samples, and $\sim$ means distributed as. The ambient white noise can be reduced by simple averaging of multiple A-scans for the same scanned volume. Second, the clutter noise $c[n]$ is caused by the material microstructure, and is assumed to be a colored Gaussian noise process with a $\mathrm{PDF} \mathbf{c} \sim \mathcal{N}\left(\mathbf{0}, \mathbf{C}_{c}\right)$, where $\mathbf{C}_{c}$ is the Toeplitz autocorrelation matrix of the clutter. The clutter noise $c[n]$ and the impulse response $h[n]$ will be explored in more detail in section 2.A. We note that the use of the discrete time representation indicates that the real continuous signals have been sampled with a sampling rate $F_{s}=\frac{1}{T_{s}} \mathrm{~Hz}$. In addition, we described all signals as a function of time, while its relationship to spatial distance is understood through the equation $\mathrm{c}=$ distance(meter) / time(second), where $\mathrm{c}$ is the speed of sound in the material.

\subsubsection{Random LTI Filter $\mathrm{h}[\mathrm{n}]$ and Clutter Noise $\mathrm{c}[\mathrm{n}]$}

In this subsection, we show how to construct a new physically motivated model for the grain scattering as a Gaussian WSS random process, and determine its mean, autocorrelation sequence (ACS), and its power spectral density (PSD). The process $h[n]$ can be thought of as the product of two independent processes $a[n]$, and $u\left[n-m_{0}\right]$ (normalized) that is $h[n]=a[n]\left(u\left[n-m_{0}\right]-\mu_{u}\right)$ (it can be shown that the product of two independent WSS processes is also WSS [19, p.591]). A portion of a sample realization of $a[n], u[n], u\left[n-m_{0}\right]$, and $h[n]$ is shown in Fig.

2. The amplitude variations of the reflected energy from the grains depend on many factors, such as grain size, orientation, shape, distance from the transducer, the transmitted pulse characteristics, and acoustic impedance. Such dependency makes these variations very random and are best described as a random process. 
The process $a[n]$ is assumed to be an IID Gaussian random process with a PDF $\mathbf{a} \sim \mathcal{N}\left(\mathbf{0}, \sigma_{A}^{2} \mathbf{I}\right)$. This is a practical assumption also used in [15], [16]. The process $u[n]$ is an impulse train process with impulses that are randomly distributed as described in the following paragraph. Refer to Fig. 2, without loss of generality, let $I_{i}=\{n: i M \leq n \leq i M+(M-1)\}$. Then:

1. In each interval $I_{i}$, a position is chosen at random for the spike. That is to say $\xi_{i} \in[0, M-1]$, where $\xi_{i}$ is a discrete random variable taking on integer values with $\xi_{i} \sim \mathcal{U}[0, M-1]$, and $\mathcal{U}$ denotes the discrete uniform distribution.

2. All spike locations $\xi_{i}$ 's are $I I D$. This forms the process $u[n]$.

3. Then the process $u[n]$ is shifted to the right by $m_{0}$ samples, where $m_{0} \sim$ $\mathcal{U}[0, M-1]$.

4. The random shift $m_{0}$ is independent of all $\xi_{i}$ 's, which leads to our new process $v[n]=u\left[n-m_{0}\right]$.

Since in practice the transducer width is much larger than a single grain size, we expect to see several overlapped spikes that come from all directions and violate the single spike per average grain size assumption. However, such an assumption is an important simplification that led to a deterministic robust model that performed well on real ultrasonic data as seen in section 4. Moreover, the simplification of assuming one spike per average grain size can be thought of as if the axial distance between the transducer and the scanned volume is divided into small equally spaced sections (that is the average grain size) and we only allow one reflection per average grain size and the reflection represents the net results to all the reflections that arrive at that particular distance/time. As discussed before, many models assume that the scatterer delays are uniformly distributed over the total scanned volume, to account for the random orientation, shape, and size of the grains [12], [17], [18]. 

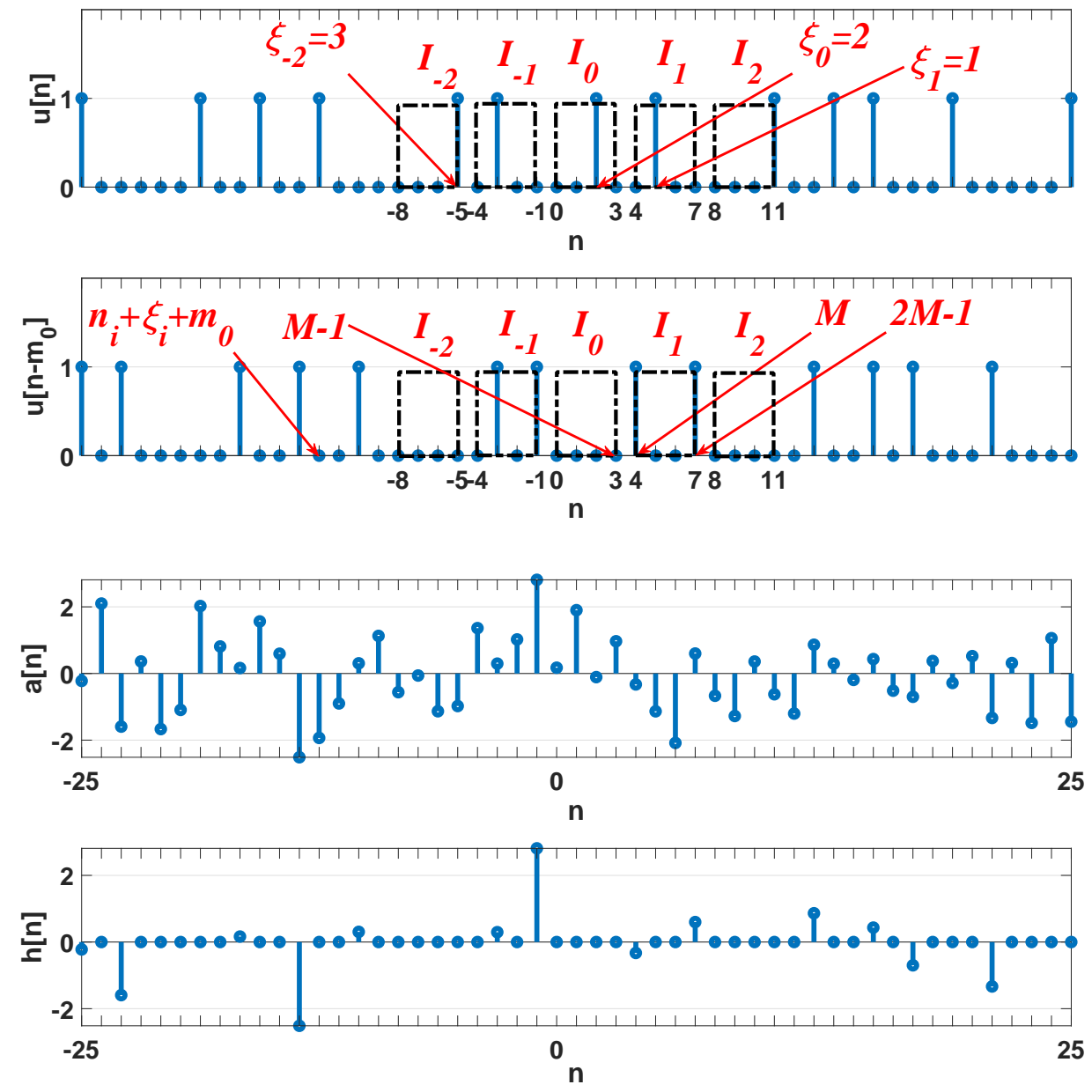

Figure 2: Realization portion of $u[n], u\left[n-m_{0}\right], a[n]$, and $h[n]=a[n]\left(u\left[n-m_{0}\right]-\frac{1}{M}\right)$ $\left(M=4\right.$ and $\left.m_{0}=2\right)$

However, simulating uniform distributed delays over the total scanned volume will result in many improbable and physically impossible situations, such as having three or more scatterering centers less than an average grain size apart. On the other hand, our model incorporates the single scatterer restriction per average grain size and utilizes the average grain size explicitly (refer to assumption 1). It also accounts for the random shape and orientation of the grains (refer to assumptions 1 and 3). Assumption 3 is a necessary step to transfer the process $h[n]$ from being a non-stationary to a WSS process [20, p.135]. Referring to assumptions $1-3, M$ is a 
parameter that represents a number of samples, and is proportional to the average grain size of the material under testing, as well as the sampling rate. Assume the distance between the surface of the transducer and a certain grain is $\mathcal{L}$ meter. In an NDT testing session, the sound will travel from the surface of the transducer to the first edge (boundary) of that grain and bounce back, and the total roundtrip distance traveled will be $2 \mathcal{L}$ meter. Next, the distance traveled from the surface of the transducer to the second edge is $2(\mathcal{L}+D)$ meter, where $D$ is the average grain size in meter, and therefore the difference in the distance that these two reflections will have in a typical A-scan is $2 D$ meter. As an example, if we have a material with an average grain size of $D=100 \mu \mathrm{m}$, a sampling rate of $F_{s}=100 \mathrm{MHz}$, and the speed of sound in the material is $\mathrm{c}=6000 \mathrm{~m} / \mathrm{sec}$, then using (2) we get $M=4$, where \lceil\rceil indicates the round up to the nearest integer, and results from

$$
M=\left\lceil\frac{2 D}{c T_{s}}\right\rceil
$$

By making $M=4$ for the previous example, it allows only one scatterer center per average grain size, which emphasizes the single scatterer assumption (Rayleigh region). The random shift $\xi_{i}$ accounts for the different orientation and shape of the grains; therefore the scatterer center will vary from grain to grain.

As for the clutter $c[n]$, the input to the random filter $h[n]$ is assumed to be the transmitted pulse $s[n]$, then $c[n]=s[n] * h[n]$, where $*$ denotes the convolution operator. Since the convolution is a commutative operation, we can write $c[n]=$ $h[n] * s[n]$, where the filter input is now $h[n]$ and the filter impulse response is $s[n]$. Since we assumed $h[n]$ is a WSS Gaussian random process, let us denote its distribution by $\mathcal{N}\left(\mathbf{0}, \mathbf{C}_{h}\right)$, where $\mathbf{C}_{h}$ is the Toepltiz autocorrelation matrix. We know that the distribution of $c[n]$ will also be a WSS Gaussian random process with ACS equal to

$$
r_{c}[k]=s[-k] * s[k] * r_{h}[k]
$$


and PSD equal to

$$
P_{c}(f)=|S(f)|^{2} P_{h}(f)
$$

where $-\frac{1}{2} \leq f \leq \frac{1}{2}, r_{h}[k]$ and $P_{h}(f)$ are the ACS and PSD for the process $h[n]$, respectively [20, p.602]. A full derivation of the mean, ACS, and PSD of $h[n]$ is given in the Appendix. Letting $\alpha=\frac{1}{M^{2}}(M-1) \sigma_{A}^{2}$, then the mean, ACS, and PSD of $h[n]$ is given by $\mu_{h}=0, r_{h}[k]=\alpha \delta[k], P_{h}(f)=\alpha$ and for $c[n]$ using (3) and (4), we get

$$
r_{c}[k]=\alpha(s[-k] * s[k])
$$

and a PSD of

$$
P_{c}(f)=\alpha|S(f)|^{2} \quad-\frac{1}{2} \leq f \leq \frac{1}{2}
$$

Thus, we can denote the distribution of $\mathbf{c}$ as $\mathcal{N}\left(\mathbf{0}, \mathbf{C}_{c}\right)$, where the elements of $\mathbf{C}_{c}$ can be calculated from $\left[\mathbf{C}_{c}\right]_{i j}=\alpha[s[k] * s[-k] * \delta[k]]_{k=i-j}$. As shown in (5), the clutter matrix $\mathbf{C}_{c}$ depends only on two parameters $\sigma_{A}^{2}$ and $M$ (for a given transmitted ultrasonic signal $s[n])$. As indicated, the process $h[n]$ has a flat spectrum so the output PSD $P_{c}(f)$ of the random LTI system is just a scaled version of the energy spectral density (ESD) of the transmitted pulse $s[n]$ (shown in (6)). Moreover, the Gaussian assumption for the clutter $c[n]$ is a reasonable one when the material is homogenous (which we assumed) and the number of grains is large enough for the central limit theory to hold [21].

The non-stationary behavior of the clutter arises from the distance-dependent attenuation, frequency-dependent attenuation, absorption, and diffraction [17]. Even though the non-stationary mean and autocorrelation functions completely characterize the process, the analytical derivation of these statistics is demanding [11], and will not be considered here, though an attempt has been made in [22]. To compromise, we simplified the problem to be a WSS process by not taking the distance-dependent and frequency-dependent attenuation into account. How- 
ever, it was determined that, for a short-time data window, any non-stationarities due to material attenuation is small and can be ignored [13]. Similar to speech processing, a window of 10-20 msec of the data can be assumed stationary even though the process is heavily non-stationary [23]. This is not the case for the nonstationarity caused by the transducer focusing effect [13]. Hence, we focused our

simulation on the far field area, which starts at approximately $\frac{d^{2}}{4 \lambda}$ meters, where $d$ is the transducer diameter in meters, and $\lambda$ is the wavelength of the transmitted pulse in meters [24]. An adaptive implementation is possible using our model, by segmenting the data into smaller time windows and then applying the model to each segment. What makes employing our model different than other similar adaptive implementations [17], [10], is that even by segmenting the data, only one parameter needs to be estimated for each segment, that is $\sigma_{A}^{2}$, in comparison to others, where a full covariance matrix (row) needs to be estimated for each segment. Here, the trade-off is between complexity and non-stationarity on one hand and simplicity and WSS on the other.

\subsection{Detector Implementation and Comparison 1.3.1 Matched Filter (MF) Vs Generalized Matched Filter (GMF)}

To show the advantage of using our model, it is important to compare it with other commonly used methods. However, the lack of a unified database that can be used to compare performances and the use of different metrics to evaluate these performances, made the comparison a difficult one. In addition, the lack of appropriate test specimens has made the development and evaluation of detection techniques even more challenging [13]. For the above reasons it was difficult to compare the performance of our model with other methods. Nevertheless, in [13] it was shown that the use of the colored Gaussian model for the clutter was more consistent than the split spectrum method [10], [11]. Interestingly enough, our 
model implementation leads to an easily implemented optimal detector, under the assumption of a known defect signal $A_{0} s\left[n-n_{0}\right]\left(A_{0}\right.$ and $n_{0}$ are known). A comparison of our model with the conventional matched filter, which is used heavily in similar applications, also in radar and sonar, was completed to show its advantage. Matched filtering uses only the diagonal part of the Toeplitz autocorrelation matrix (assuming the process is normalized i.e. has zero mean), and thus the noise is considered white. To start we assume that the signal model is known, the observation window is aligned with the actual defect location $\left(n_{0}\right.$ is known), and the amplitude is known and positive $\left(A_{0}>0\right.$ and known). In this manner, the problem becomes a classical known deterministic signal in colored Gaussian noise. For this problem, the optimal detector that maximizes the probability of detection for a fixed probability of false alarm, can be obtained by using the Neyman-Pearson criterion [25]. Referring to Fig. 1 and (1), the optimal detector decides a defect is present $\left(\mathcal{H}_{1}\right)$ if $L_{G}(\mathbf{x})=\frac{p\left(\mathbf{x} ; \mathcal{H}_{1}\right)}{p\left(\mathbf{x} ; \mathcal{H}_{0}\right)}>\gamma$, where $L_{G}(\mathbf{x})$ is the likelihood ratio function, $p\left(\mathbf{x} ; \mathcal{H}_{1}\right)$ and $p\left(\mathbf{x} ; \mathcal{H}_{0}\right)$ are the probability density functions under $\mathcal{H}_{1}$ and $\mathcal{H}_{0}$ respectively, and $\gamma$ is a threshold constant. We have

$$
L_{G}(\mathbf{x})=\frac{\frac{1}{(2 \pi)^{\frac{N}{2}}\left|\mathbf{C}_{c}+\sigma_{w}^{2} \mathbf{I}\right|^{\frac{1}{2}}} \exp \left[-\frac{1}{2}(\mathbf{x}-\mathbf{s})^{T}\left(\mathbf{C}_{c}+\sigma_{w}^{2} \mathbf{I}\right)^{-1}(\mathbf{x}-\mathbf{s})\right]}{\frac{1}{(2 \pi)^{\frac{N}{2}}\left|\mathbf{C}_{c}+\sigma_{w}^{2} \mathbf{I}\right|^{\frac{1}{2}}} \exp \left[-\frac{1}{2} \mathbf{x}^{T}\left(\mathbf{C}_{c}+\sigma_{w}^{2} \mathbf{I}\right)^{-1} \mathbf{x}\right]}
$$

where $\mathbf{s}=\left[\begin{array}{llll}A_{0} s\left[n_{0}\right] & A_{0} s\left[n_{0}+1\right] & \cdots & A_{0} s\left[n_{0}+L-1\right]\end{array}\right]^{T}$, and $L$ is the number of samples that corresponds to the transmitted pulse length. Let $\mathbf{R}=\mathbf{C}_{c}+\sigma_{w}^{2} \mathbf{I}$. After simplifying the above expression we get

$$
T(\mathbf{x})=\mathbf{x}^{T} \mathbf{R}^{-1} \mathbf{s}_{0}
$$

where $T(\mathbf{x})$ is called the test statistic, and $\mathbf{s}_{0}=$ $\left[s\left[n_{0}\right] \quad s\left[n_{0}+1\right] \quad \cdots \quad s\left[n_{0}+L-1\right]\right]^{T}$. This form of the test statistic is called the generalized matched filter (we will call it $T_{G M F}(\mathbf{x})$ ) [25], since we are 
correlating the received signal $\mathbf{x}$ with a whitening version $\mathbf{R}^{-1} \mathbf{S}$ of the transmitted pulse. As discussed before, the difference between the MF and the GMF is the use of the off diagonal terms in $\mathbf{R}$. For the matched filter the test statistic becomes the following

$$
T_{M F}(\mathbf{x})=\frac{\mathbf{x}^{T} \mathbf{s}_{0}}{\sigma_{R}^{2}}
$$

where $\sigma_{R}^{2}$ is the diagonal element of the matrix $\mathbf{R}$. Since we assumed all parameters are known, we can move the $\sigma_{R}^{2}$ to the threshold part of the test and the new test statistic becomes $T_{M F}(\mathbf{x})=\mathbf{x}^{T} \mathbf{s}_{0}$. The distributions of the test statistics for both the GMF and MF are the following [25]

$$
T_{G M F}(\mathbf{x}) \sim \begin{cases}N\left(0, \mathbf{s}_{0}^{T} \mathbf{R}^{-1} \mathbf{s}_{0}\right) & \text { under } \mathcal{H}_{0} \\ N\left(A_{0} \mathbf{s}_{0}^{T} \mathbf{R}^{-1} \mathbf{s}_{0}, \mathbf{s}_{0}^{T} \mathbf{R}^{-1} \mathbf{s}_{0}\right) & \text { under } \mathcal{H}_{1}\end{cases}
$$

and

$$
T_{M F}(\mathbf{x}) \sim \begin{cases}N\left(0, \mathbf{s}_{0}^{T} \mathbf{R} \mathbf{s}_{0}\right) & \text { under } \mathcal{H}_{0} \\ N\left(A_{0} \mathbf{s}_{0}^{T} \mathbf{s}_{0}, \mathbf{s}_{0}^{T} \mathbf{R} \mathbf{s}_{0}\right) & \text { under } \mathcal{H}_{1}\end{cases}
$$

For these test statistics the performance for the GMF is

$$
\begin{gathered}
P_{F A}=Q\left(\frac{\gamma^{\prime}}{\sqrt{\mathbf{s}_{0}^{T} \mathbf{R}^{-1} \mathbf{s}_{0}}}\right) \\
P_{D}=Q\left(Q^{-1}\left(P_{F A}\right)-A_{0} \sqrt{\mathbf{s}_{0}^{T} \mathbf{R}^{-1} \mathbf{s}_{0}}\right)
\end{gathered}
$$

where the threshold can be found as $\gamma^{\prime}=Q^{-1}\left(P_{F A}\right) \sqrt{\mathbf{s}_{0}^{T} \mathbf{R}^{-1} \mathbf{s}_{0}}, P_{F A}$ is the probability of false alarm, $P_{D}$ is the probability of detection, and $Q()$ is the right-tail probability of standard normal Gaussian. For the MF, it is

$$
\begin{gathered}
P_{F A}=Q\left(\frac{\gamma^{\prime \prime}}{\sqrt{\mathbf{s}_{0}^{T} \mathbf{R} \mathbf{s}_{0}}}\right) \\
P_{D}=Q\left(Q^{-1}\left(P_{F A}\right)-A_{0} \sqrt{\frac{\left(\mathbf{s}_{0}^{T} \mathbf{s}_{0}\right)^{2}}{\mathbf{s}_{0}^{T} \mathbf{R s}_{0}}}\right)
\end{gathered}
$$

where the threshold can be found as $\gamma^{\prime \prime}=Q^{-1}\left(P_{F A}\right) \sqrt{\mathbf{s}_{0}^{T} \mathbf{R} \mathbf{s}_{0}}$ for the MF. From (7) and (8) the probability of detection, for a given probability of false alarm, depends on $d_{G M F}^{2}=A_{0} \sqrt{\mathbf{s}_{0}^{T} \mathbf{R}^{-1} \mathbf{s}_{0}}$ for the GMF, and $d_{M F}^{2}=A_{0} \sqrt{\frac{\left(\mathbf{s}_{0}^{T} \mathbf{s}_{0}\right)^{2}}{\mathbf{s}_{0}^{T} \mathbf{R s}_{0}}}$ for the 
MF, where $d_{G M F}^{2}$ and $d_{M F}^{2}$ are called the deflection coefficients [25]. To quantify the advantage of using our model over the usual MF, we can study the following ratio (which can be interpreted as the signal to noise ratio gain of the GMF over $\mathrm{MF})$

$$
S N R_{\text {Gain }}=\frac{d_{G M F}^{2}}{d_{M F}^{2}}=\frac{\mathbf{s}_{0}^{T} \mathbf{R}^{-1} \mathbf{s}_{0} \mathbf{s}_{0}^{T} \mathbf{R} \mathbf{s}_{0}}{\left(\mathbf{s}_{0}^{T} \mathbf{s}_{0}\right)^{2}}
$$

where we will use $10 L O G 10\left(S N R_{\text {Gain }}\right) \mathrm{dB}$ in accordance with convention. The above ratio is always greater than 1 . To make a qualitative comparison, we computed the autocorrelation matrix $\mathbf{R}=\mathbf{C}_{c}+\sigma_{w}^{2} \mathbf{I}$ for two different scenarios. First, where the clutter noise dominates the ambient white noise and second, where the ambient white noise dominates the clutter noise. Let $C N R(d B)=$ $10 L O G 10\left(\frac{r_{c}[0]}{r_{w}[0]}\right)$, where $r_{c}[0]$ is the total average power of the clutter noise and $r_{w}[0]$ is the total average power of the ambient white noise. We know that $r_{c}[0]=\alpha \mathcal{E}_{s}$ and $r_{w}[0]=\sigma_{w}^{2}$, where $\mathcal{E}_{s}$ is the energy of the transmitted ultrasonic pulse and $\alpha=\frac{1}{M^{2}}(M-1) \sigma_{A}^{2}$. The term $C N R(\mathrm{~dB})$ is what is commonly called clutter to ambient noise ratio. The quantity $C N R(\mathrm{~dB})$ indicates which noise is dominating the overall noise for a given transmitted pulse. Even though the matrix $\mathbf{R}$ also depends on the energy of the transmitted pulse $s[n]$, as mentioned before, we will not pursue that in this work and, without loss of generality in computing (9), we can assume $\mathcal{E}_{s}=1$. It is clear from Fig. 3 that the performance of the GMF surpasses the $\mathrm{MF}$ as the ratio $C N R$ increases, which translates into the first scenario where the clutter noise dominates the ambient white noise. As for the second scenario, where the ambient white noise dominates the clutter noise, it is shown in Fig. 3 that the improvement in performance is less, as expected. However our detector performance always surpasses the MF. Note that in NDT application the $C N R$ usually falls in the region shown in Fig. 3 to the right so we expect an advantage of almost $10 \mathrm{~dB}$ for using the GMF over the MF. Next, the receiver operating 


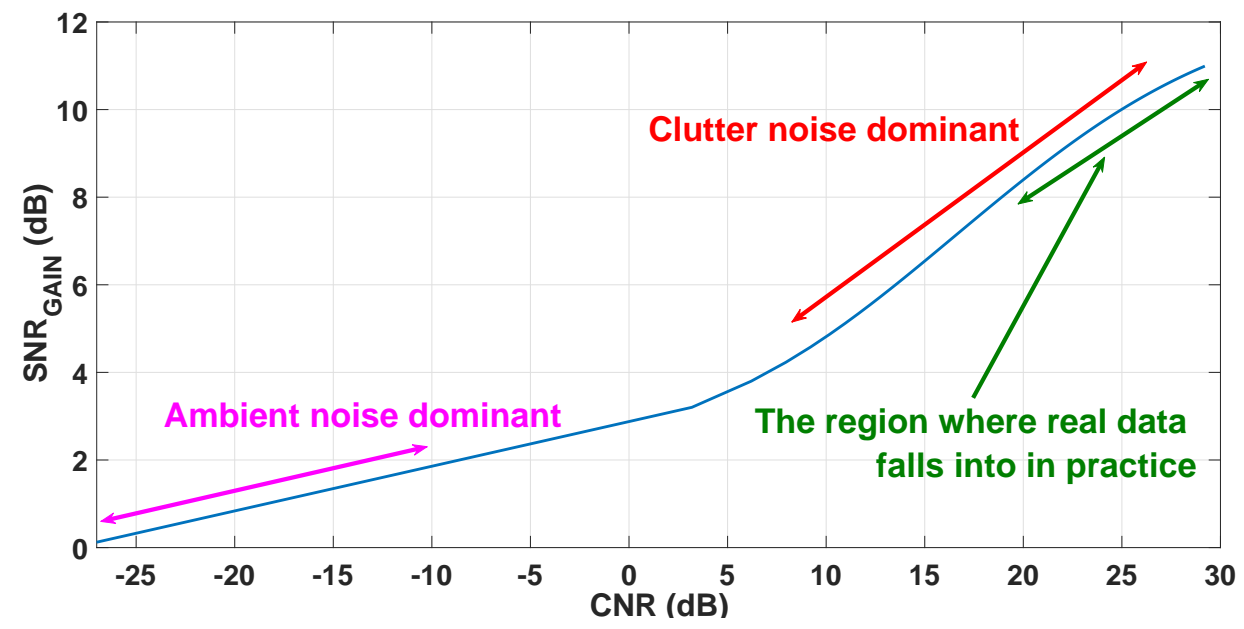

Figure 3: The signal to noise gain $S N R_{\text {Gain }}$ in $\mathrm{dB}$ vs clutter to ambient noise $(\mathrm{CNR})$ in $\mathrm{dB}$

characteristics (ROC) curve is used as another evaluation tool. We chose the following parameters $\sigma_{A}^{2}=0.035, \sigma_{w}^{2}=0.0001$ (these values were chosen to match the real data). The values of the amplitude reflect cases from low signal to noise ratio (SNR) to high SNR. Evidently, our model shows a significant performance advantage in the low SNR region and it improves as the SNR increases as shown in Fig. 4.

\subsubsection{Practical Detector Implementation-GLRT}

To apply the detector to the problem at hand, since we need to estimate $-\infty<A_{0}<\infty$ and $n_{0}$. In practice, we have two approaches, the generalized likelihood ratio test (GLRT) approach and the Bayesian approach. The GLRT appears to be more widely used for its ease of implementation and less restrictive assumptions [25], and it will be the approach we follow here. The detector that works well in practice and usually maximizes the probability of detection for a given probability of false alarm for such a problem can be achieved by implementing the GLRT, which decides $\mathcal{H}_{1}$ if $\ln \left(L_{G}(\mathbf{x})\right)=\ln \left(p\left(\mathbf{x}, \hat{A}_{0}, \hat{n}_{0} ; \mathcal{H}_{1}\right) / p\left(\mathbf{x} ; \mathcal{H}_{0}\right)\right)>\ln (\gamma)$, where $\ln$ indicates the natural logarithm (a monotonically increasing function), $\hat{A}_{0}$ 

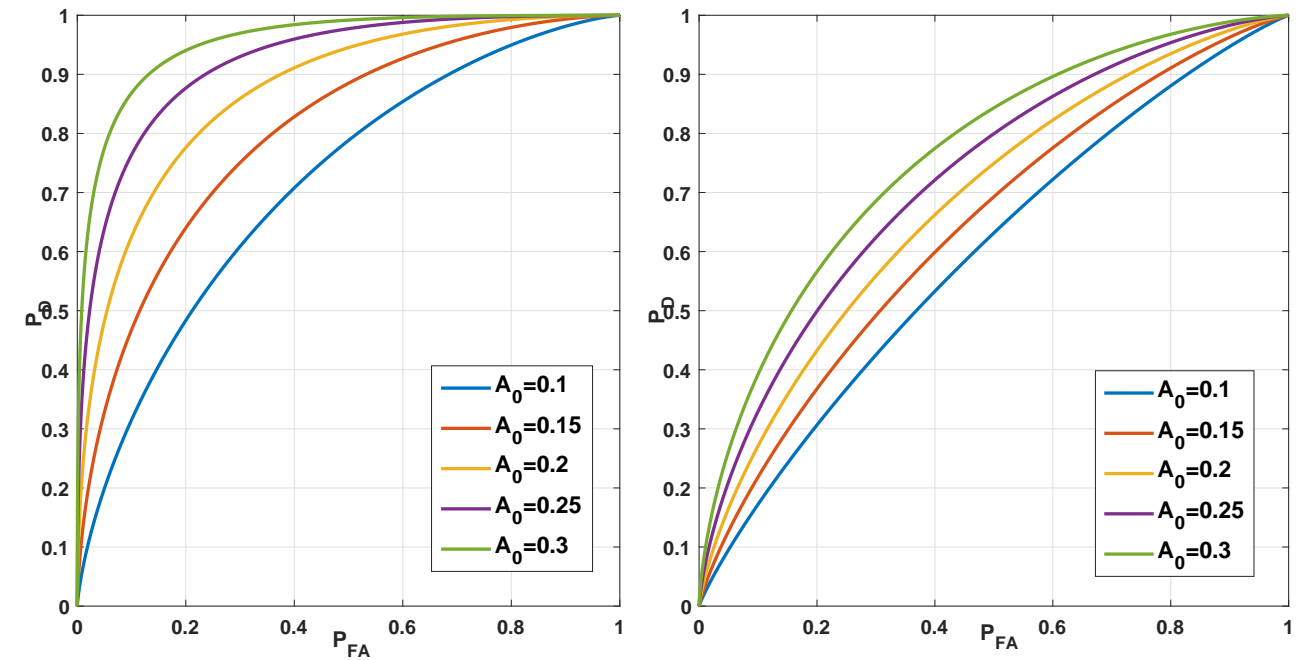

Figure 4: ROC curves for both GMF (left) and MF (right) for different $A_{0}$

and $\hat{n}_{0}$ is the maximum likelihood estimator for $A_{0}$ and $n_{0}$, respectively [26]. For that we get the following results

$$
\begin{aligned}
& \ln L_{G}(\mathbf{x})=\ln \frac{p\left(\mathbf{x} ; \hat{A} ; \hat{n}_{0}, \mathcal{H}_{1}\right)}{p\left(\mathbf{x} ; \mathcal{H}_{0}\right)} \\
& \ln L_{G}(\mathbf{x})=\max _{n_{0} \in[0: N-L]}\left[\frac{\left(\mathbf{x}^{T} \mathbf{R}^{-1} \mathbf{s}_{0}\right)^{2}}{\mathbf{s}_{0}^{T} \mathbf{R}^{-1} \mathbf{s}_{0}}\right] .
\end{aligned}
$$

We decide $\mathcal{H}_{1}$ (a defect is present) if

$$
T(\mathbf{x})=\max _{n_{0} \in[0: N-L]} \frac{\left(\mathbf{x}^{T} \mathbf{R}^{-1} \mathbf{s}_{0}\right)^{2}}{\mathbf{s}_{0}^{T} \mathbf{R}^{-1} \mathbf{s}_{0}}>\gamma^{\prime} .
$$

Note to get (10) we used the MLE for the amplitude $\hat{A}_{0}=\frac{\mathbf{x}^{T} \mathbf{R}^{-1} \mathbf{s}_{0}}{\mathbf{s}_{0}^{T} \mathbf{R}^{-1} \mathbf{s}_{0}}$. To get the theoretical detection performance of the above test statistic is a difficult task, since we need to determine the PDF of the maximum of $N-L+1$ correlated Gaussian random variables and will not be pursued here. The interested reader can refer to [27], [25, p.260] for more insight on how to obtain such a PDF. The option to determine the performance by simulation is a practical one and will be followed here. We next summarize the steps to implement our detector

1. Estimate $\sigma_{w}^{2}$. This can be done in the frequency domain, if a backwall signal is available or could be attained empirically. 
2. Estimate $\sigma_{A}^{2}$. This can be done empirically by fitting the simulated noise to the measured noise [15],[16]. If $M$ is unknown, we can use the same empirical fitting. Our analysis showed that this is not a difficult task. In addition, our model exhibited robustness even if those parameters were misspecified.

3. After estimating $\sigma_{A}^{2}, \sigma_{w}^{2}$, and $M$, we can generate our clutter matrix $\mathbf{C}_{c}$, then construct the matrix $\mathbf{R}$ and take its inverse to get $\mathbf{R}^{-1}$.

4. Next we are ready to implement the GLRT. We estimate $n_{0}$ by using a grid search for $\hat{n}_{0}$ (using a sliding window).

5. Finally we use (10) and compare the test statistic to a threshold, which is application dependent.

In estimating $n_{0}$ we can write the sliding window samples as follows $\mathbf{s}_{\xi}=$ $\left[\begin{array}{lllll}s[\xi] & s[\xi+1] & \cdots & s[\xi+L-1]\end{array}\right]^{T}$, where $\xi \geq 0$ represents the amount of shift applied to the transmitted signal. Keep in mind that the increment for which $\xi$ can have depends on the shape of the transmitted signal, which we will cover in future work, and the sampling rate. However, the sampling rate in such applications is considered high enough and usually will not cause a problem. A poor choice of the increment of $\xi$ will lead to a degradation of the detector performance since we will miss the maximum correlation point between the deterministic known transmitted signal and the unknown defect reflection signal. In our simulation, an increment of one sample was adopted since the length of the data record was reasonable. If the data record is long, a larger increment with an overlapping window will be more computationally efficient.

In this work, we determined the threshold by simulation, since the real test statistic PDF under $\mathcal{H}_{0}$ could not be achieved analytically. In practice, samples of defect free blocks can be first used to get an estimate for the PDF under $\mathcal{H}_{0}$. 


\subsection{Experimental results with Real Data}

The A-scans were obtained from 2-in diameter type 303 austenitic stainless steel cylindrical rods. The rods were heat treated for one hour at temperatures of $1350^{\circ} \mathrm{C}, 1370^{\circ} \mathrm{C}$, and $1387^{\circ} \mathrm{C}$ to obtain various grain sizes. In each case the heat treated samples were put out rapidly in water. After that the samples were prepared to simulate a suitable flat-bottom holes. The initial holes had to be made by means of an electro-discharge machine and later enlarged by drilling. The hole dimension were selected so that the reflected ultrasonic signal from both the grain and the flaw would be the same. In order to determine the average grain size sections were cut along the diameter of the heat treated samples and were examine myelographically in both the axial and radial directions. The grain size of the heat treated samples were analyzed by the intercept method. The heat treated samples resulted in grain sizes of 86, 106, and $160 \mu \mathrm{m}$. To increase the accuracy of the estimates, grain count was obtained for two perpendicular line segments for each surface. We only focused our simulation on the heat treated samples with the grain size of $160 \mu \mathrm{m}$, since it is the most difficult case, and there were 4 samples. The flat-bottom hole with diameter of $4.22 \mathrm{~mm}$ was simulated at a different location from the front face of the cylindrical blocks. The locations are as follows; 29-mm, 36- $\mathrm{mm}$, 43- mm, and 29-mm. The stainless steel samples were placed in water bath and scanned with A KB-Aerotech Alpha transducer (0.5-in diameter unfocused), where the surface of the flat-bottom hole was perpendicular to the axial transducer field. The transducer has a center frequency of $5 \mathrm{MHz}$ and a Gaussian-shaped spectrum with $2.5 \mathrm{MHz}$ bandwidth. The received signals were then sampled at a sampling rate of $100 \mathrm{MHz}$. Each A-scan was repeated 200 times and then averaged to attenuate the ambient white noise. Also, the estimated speed of sound in the material was $5790 \mathrm{~m} / \mathrm{sec}$ [17]. Based on the grain size and the 

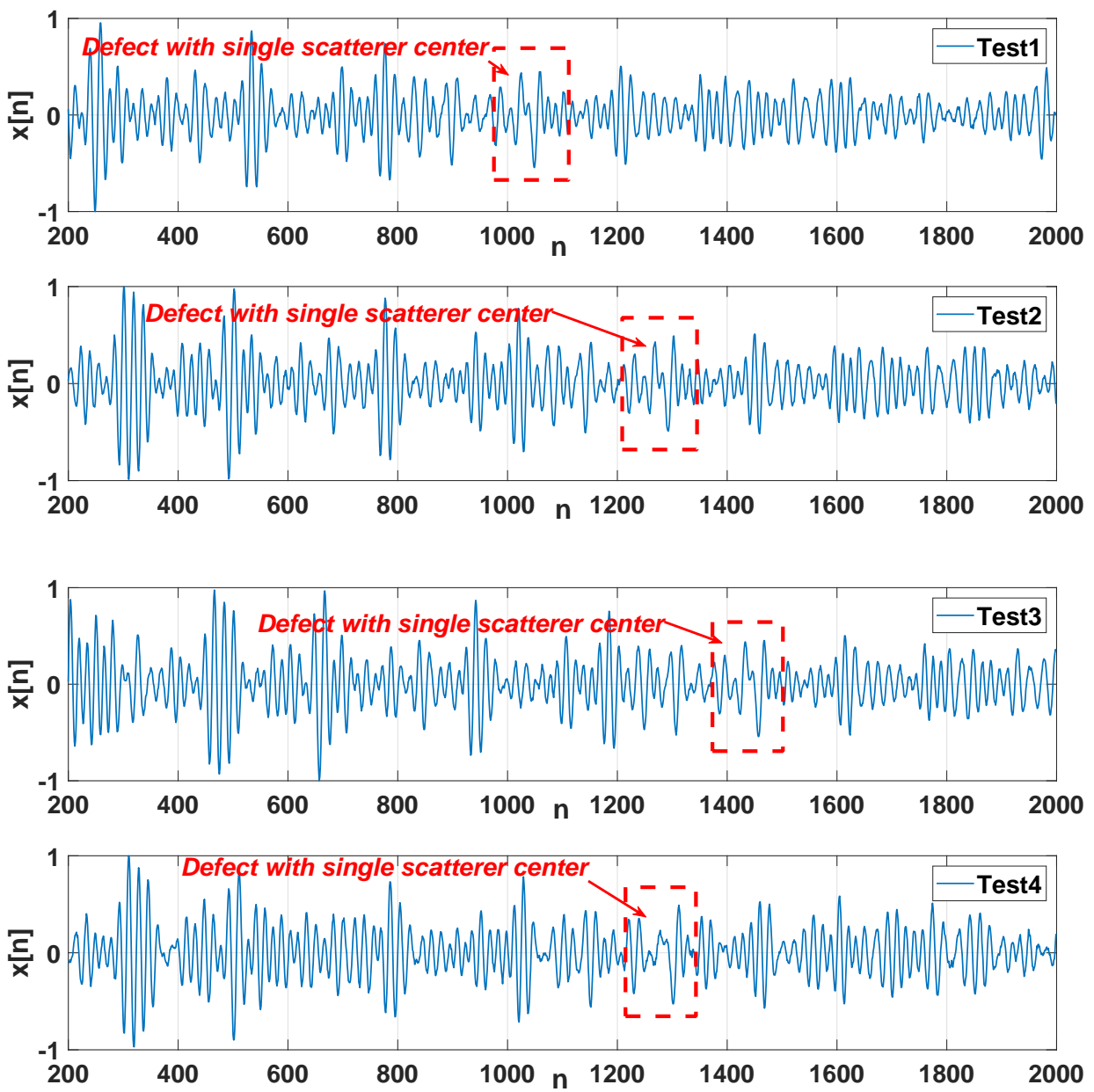

Figure 5: Received A-scan signals $x[n]$

center frequency of the transducer the scatterers fall into the two regions Rayleigh and stochastic, and hence, violate the single scatterer assumption. However, the model still performed effectively even with the deviation from the single scatter assumption. The data is shown in Fig. 5. Following the steps in section 3.B. For steps $1-2$, we got $\sigma_{w}^{2}=0.0001, \sigma_{A}^{2}=0.035$. Using (2) we got $M=6$. The transmitted pulse $s[n]$ and the spectrum of the transducer has been approximated by a Gaussian shape pulse with $5 \mathrm{MHz}$ center frequency and $2.5 \mathrm{MHz}$ bandwidth. For the duration of the transmitted pulse, we followed the same criterion as in [17]. Since the real data was the same, we got a duration that corresponds to $L=65$ 

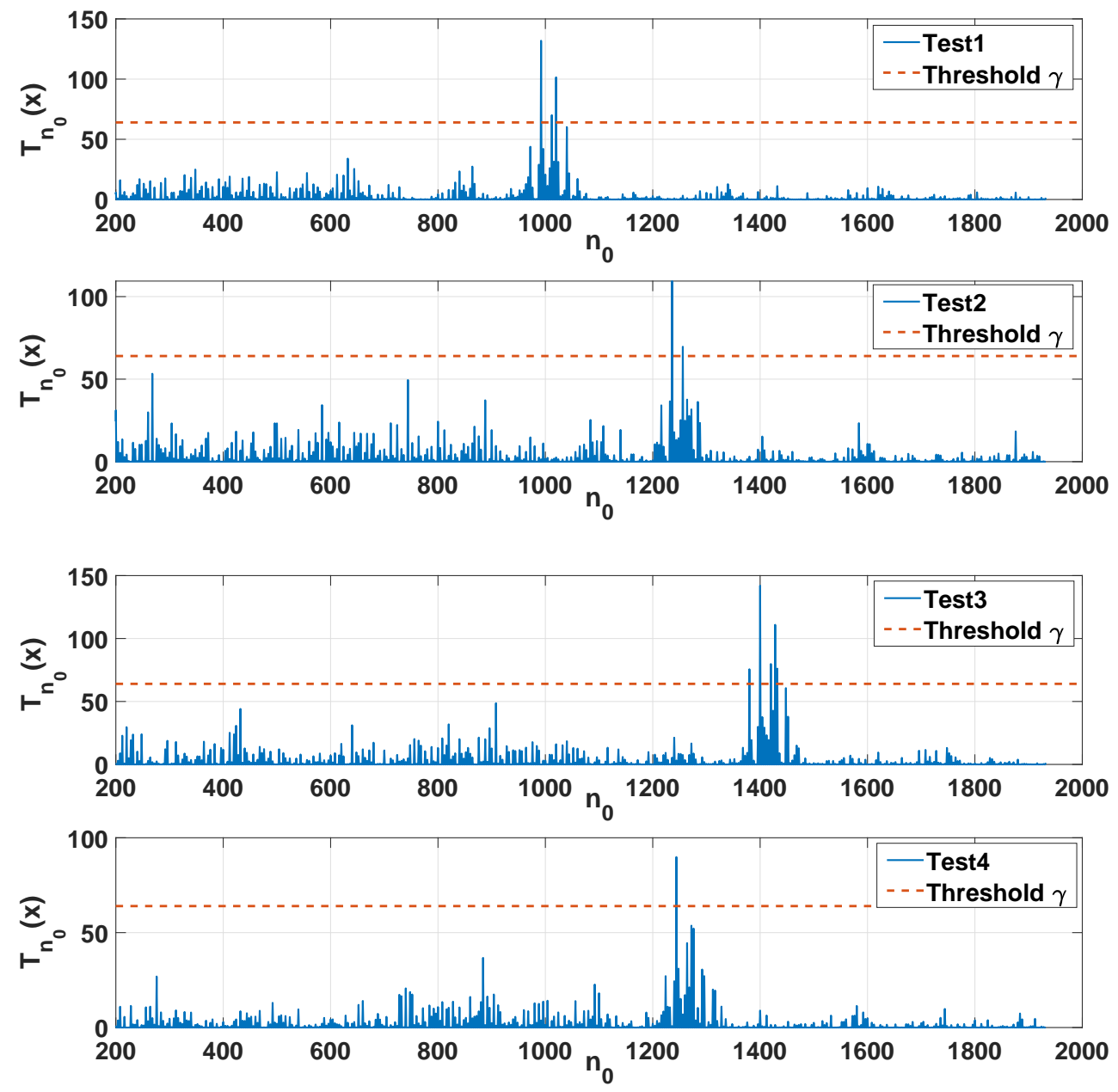

Figure 6: Test statistic $T_{n_{0}}(\mathbf{x})$ vs $n_{0}$ for GMF (GLRT Implementation)

samples at the sampling rate of $100 \mathrm{MHz}$. The phase of the transmitted pulse was set to zero, since the pulse is assumed to be Gaussian. For more details refer to $\left[17\right.$, Sec.V]. Once we had $M, \sigma_{w}^{2}$, and $\sigma_{A}^{2}$ we evaluated $\mathbf{C}_{c}$, then $\mathbf{R}$ and $\mathbf{R}^{-1}$. Next we estimated $n_{0}$ and then implemented the GLRT. Results are shown in Fig. 6, where $T_{n_{0}}(\mathbf{x})=\left.T(\mathbf{x})\right|_{n=n_{0}}=\frac{\left(\mathbf{x}^{T} \mathbf{R}^{-1} \mathbf{s}_{0}\right)^{2}}{\mathbf{s}_{0}^{T} \mathbf{R}^{-1} \mathbf{s}_{0}}$. The last step is to decide the threshold $\gamma$, which depends on the optimality criterion of interest. We simulated 1000 defect free realizations, using the estimated parameters. Next we estimated the PDF under $\mathcal{H}_{0}$ to get a threshold. We chose a threshold that gives us a $P_{F A}=0.001$, which we found to be $\gamma=64$ (red dotted line in Fig. 6). Using this threshold, we 

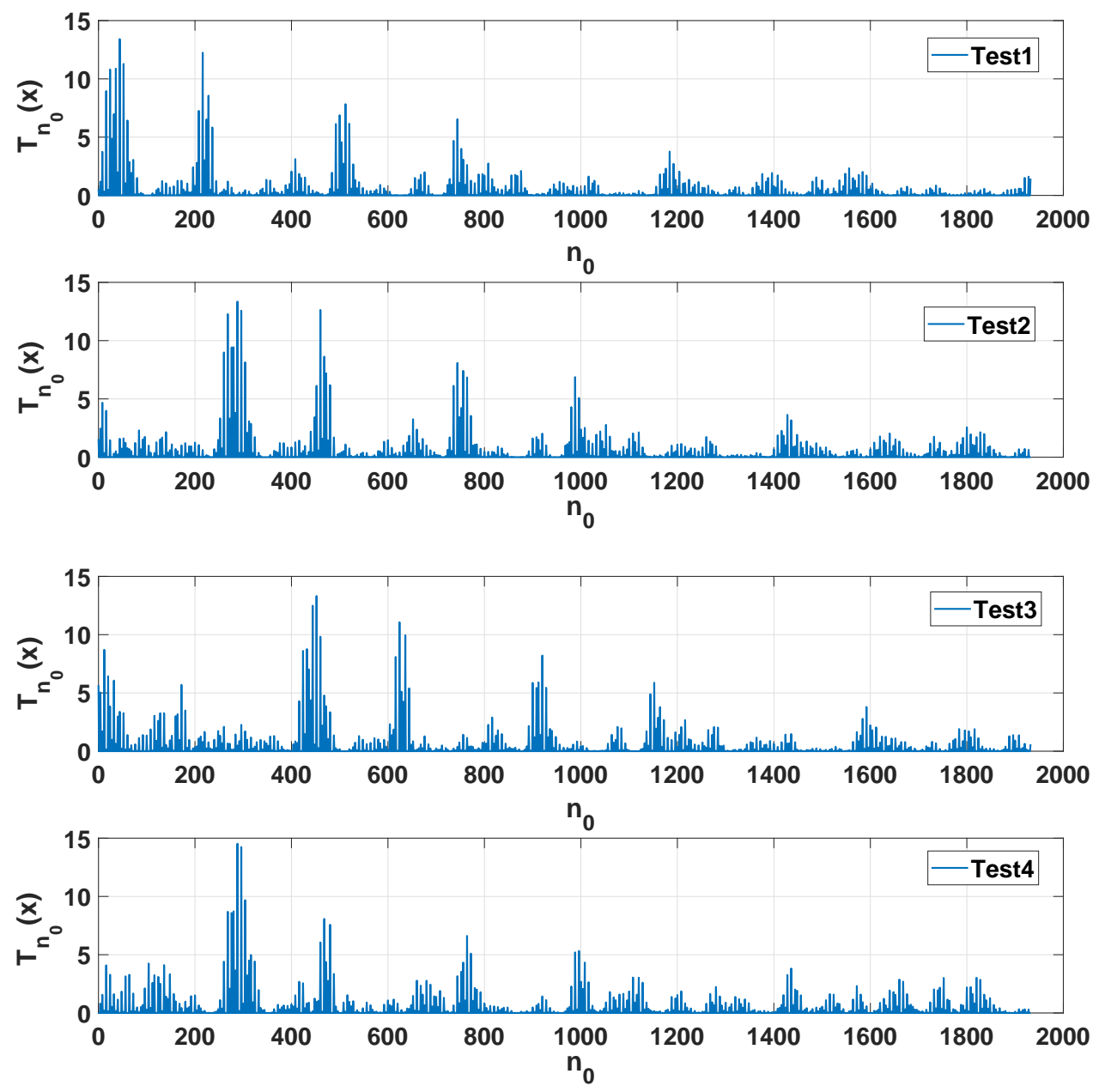

Figure 7: Test statistic $T_{n_{0}}(\mathbf{x})$ vs $n_{0}$ for MF (GLRT Implementation)

decide $\mathcal{H}_{1}$ if $T_{n_{0}}(\mathbf{x})>\gamma$ and referring back to Fig. 6 , we detected all four defects since $T_{n_{0}}(\mathbf{x})$ of the four signals was greater than $\gamma=64$. As a comparison, we show the results of the matched filter implementation in Fig. 7, which indicates that the matched filter was not able to detect any of the simulated defects.

\subsection{Parameter Sensitivity}

An advantage of using the proposed model is that even if the value of the average grain size is unknown (which indicates that the number of samples $M$ is unknown) our model is fairly robust to parameter errors. Since $M$ and $\sigma_{A}^{2}$ in this case are estimated empirically (assuming we have an estimate for the ambient 
Table 1: Test Statistics $T_{n_{0}}(\mathbf{x})$ of all four real data waveforms for different combinations of $M$ and $\sigma_{A}^{2}$

\begin{tabular}{|l|l|l|l|l|}
\hline Parameters & Test1 & Test2 & Test3 & Test4 \\
\hline$M=4, \sigma_{A}^{2}=0.027$ & $T_{n_{0}}(\mathbf{x})=126$ & $T_{n_{0}}(\mathbf{x})=105$ & $T_{n_{0}}(\mathbf{x})=136$ & $T_{n_{0}}(\mathbf{x})=87$ \\
\hline$M=8, \sigma_{A}^{2}=0.04$ & $T_{n_{0}}(\mathbf{x})=146$ & $T_{n_{0}}(\mathbf{x})=121$ & $T_{n_{0}}(\mathbf{x})=157$ & $T_{n_{0}}(\mathbf{x})=98$ \\
\hline$M=12, \sigma_{A}^{2}=0.06$ & $T_{n_{0}}(\mathbf{x})=139$ & $T_{n_{0}}(\mathbf{x})=116$ & $T_{n_{0}}(\mathbf{x})=150$ & $T_{n_{0}}(\mathbf{x})=95$ \\
\hline
\end{tabular}

white noise variance $\sigma_{w}^{2}$ ), we are expecting to see different combinations of $M$ and $\sigma_{A}^{2}$ that lead to an approximate result that match our real data. To that end, we assumed that the average grain size for the real data was unknown $(M$ is unknown) and we estimated $M$ and $\sigma_{A}^{2}$ empirically. The results are shown in Table 1, where we followed the steps in section 3.B. For each combination of $M$ and $\sigma_{A}^{2}$ the estimate of $n_{0}$ where the maximum located for all four test signals were at the same location as in Fig. 6. The maximum test statistic values are shown on the right columns of Table 1 . If we assumed that the true PDF under $\mathcal{H}_{0}$ is the one we estimated in section 4 , then it is clear from Table 1 that we were able to detect all four defects when they are compared to the threshold $\gamma=64$. An explanation for this is that the performance of our model only depends on $\alpha$, which depends on the ratio between $M$ and $\sigma_{A}^{2}$ rather than their individual values. However, to see the detector performance where $\alpha$ increases, which translate to cases when the single-scatterer assumption is no longer valid, we plotted the deflection coefficient (dB) versus the CNR (dB) as shown in Fig. 8. Note that for NDT applications the CNR usually falls in the region between 25-35 dB.

\subsection{Multiscattering for A More Complicated Target}

By following the steps in section 3.B and implementing the GLRT we got the results shown in Fig. 10. In many situations, a defect can have a very complicated geometrical shape, which causes the defect to have more than one scatterer surfaces in one resolution cell. A resolution cell refers to the volume of the scanned 


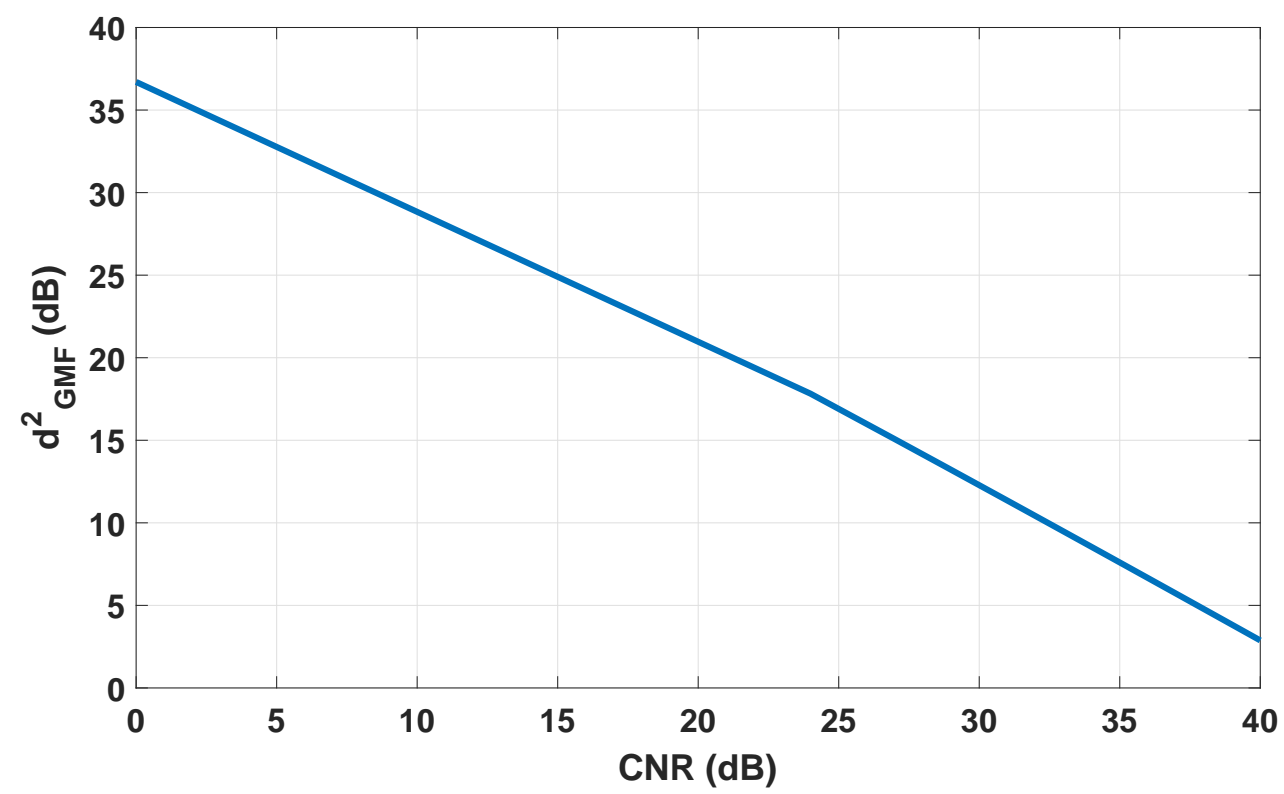

Figure 8: CNR (dB) Vs $d_{G M F}^{2}(d B)$

material, illuminated by the transducer, within which only one defect scatterer can be identified [17]. In our work, the resolution cell is assumed to be approximately equal to the transmitted ultrasonic pulse $s[n]$ duration, which corresponds to $\mathrm{L}=$ 65 samples. Since the speed of sound of the tested stainless steel material was 5790 $\mathrm{m} / \mathrm{sec}$; then the axial distance of one resolution cell is approximately $2 \mathrm{~mm}$. If the defect axial distance is contained in one resolution cell, this will cause the defect to have two or more scattering surfaces within one resolution cell. To simulate such a scenario, and since we assumed that the received signals are a distorted and delayed version of the transmitted signal, we added one additional reflected signal, which corresponds to the hypothetical second surface of the defect, to the original signals in Fig. 5. Also, white noise samples have been added and attenuation to the amplitude has been applied. The amplitude is chosen to be $50 \%$ of the estimated amplitude of the defect reflected signal that has been estimated for all four signals in section 4 . The added signal is the approximated transmitted Gaussian shape signal and the shift corresponds to the mid-point where the peak 

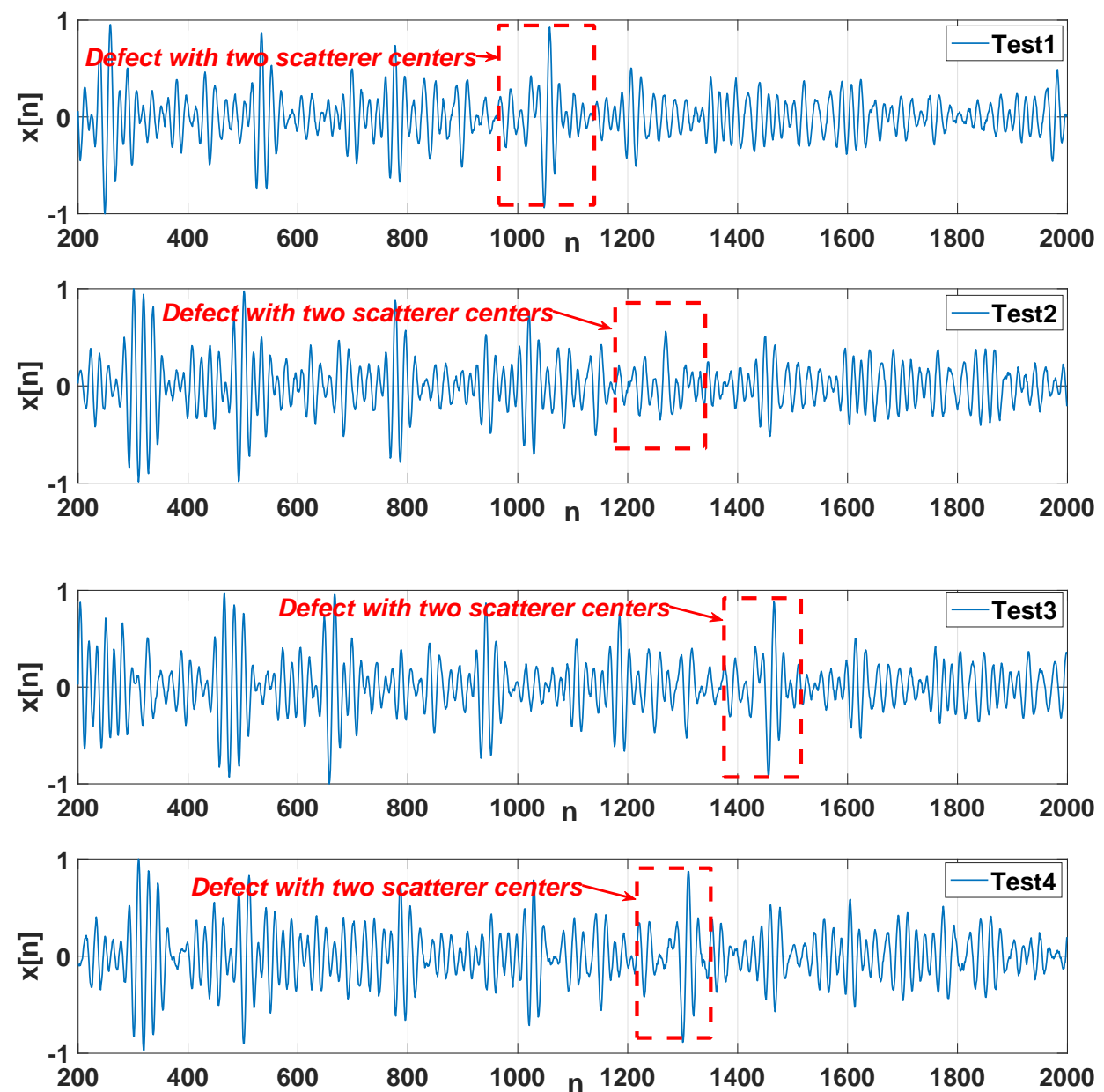

Figure 9: Received A-scan signals $x[n]$ with two scatterer centers

is located (since the Gaussian pulse is symmetric). As for the location a 1-mm distance translates to 34 samples (the speed of sound is $5790 \mathrm{~m} / \mathrm{sec}$ ). However, the location was varied to create the difficult case where the two scatterer surface reflected signals combination is of the destructive type and not the constructive type to cause the overall defect reflected signal to be attenuated. The results after adding the hypothetical simulated reflected signals from the second scatter surface are shown in Fig. 9. From Fig. 10 it is clear that the detector still worked well. It was apparent as we proceeded to simulate the same scenario but with higher amplitude levels that as long as the reflected signal from the first scattering surface 

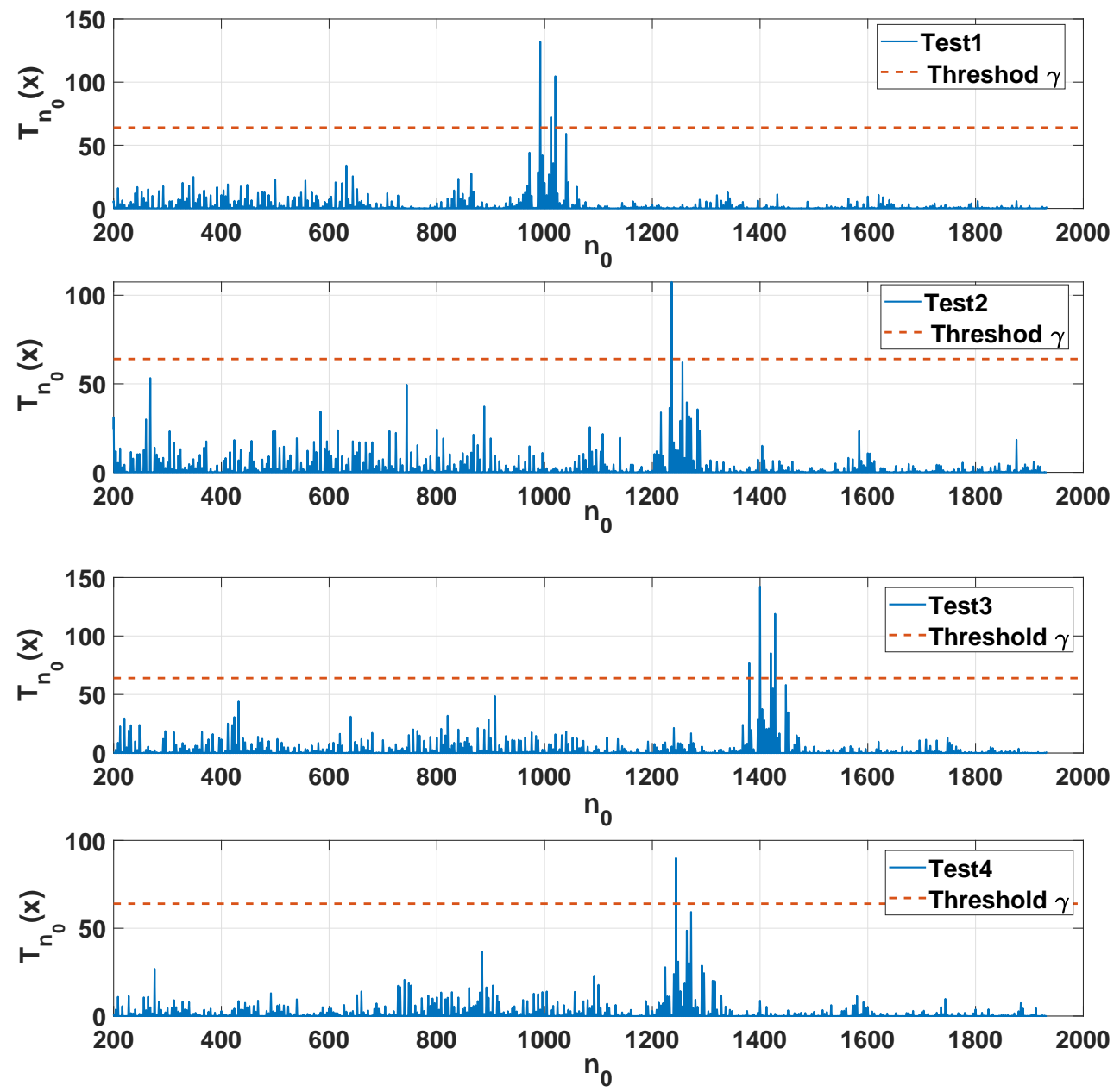

Figure 10: Test statistic $T_{n_{0}}(\mathbf{x})$ vs $n_{0}$ for the Multiscatterer case

dominates the second one, our model still worked well, even with the deviation from the single scattering assumption.

\subsection{Conclusion}

A new physically motivated model for the grain scatter noise (clutter) has been proposed and constructed to aid in the detection of a defect in the material under testing. The proposed model incorporates multiple physical characteristics such as the average grain size, random orientation and shape of the grains, and emphasizes the single scatterer assumption (Rayleigh region). It has been shown that the proposed model exhibited a large advantage over the MF by almost 10 
$\mathrm{dB}$, especially for applications where the clutter noise dominates the ambient noise. A detector implementation on real ultrasonic data has been carried out and the results indicate the excellent performance of the proposed model. Moreover, the model demonstrates a robustness towards the choice of the parameters. The simulation under the multiple scatterer assumption has been carried out and the model still led to good detectibly. It is good to keep in mind that even though the model showed excellent results on real ultrasonic data, the underlying assumption focused on the single-scatterer assumption (Rayleigh). Also, the model did not consider the distance-dependent and frequency-dependent situations, which becomes more relevant as the defect size decreases and gets closer to the grain size. Future work will focus on applying the model on defects with different sizes, geometrical shapes, and materials. Also the case of having two defects. Finally, the test on different materials that have different intrinsic properties such as inhomogeneity level, coarse level, and isotropic level need to be explored. 


\section{Appendix 1A. First and Second Order Statitics and the PSD of the Physical Model}

In Fig. 11 and 12 a spike process realization before and after the shift are shown. The intervals are defined as $I_{i}=\{n: i M \leq n \leq i M+(M-1)\}$ for all $i$. The model assumptions are stated in section 2.A. We next derive the first- and second-order PMFs.

\section{First-order PMF}

Consider an arbitrary sample point $n$ and assume that it lies in $I_{i}$ so that $n=i M+u$, where $u$ can take on integer values $0 \leq u \leq M-1$. Note that $u$ refers to the position from the start of the $i$ th interval. Then, there will be a spike at $n$ if either $i M+\xi_{i}+m_{0}=n$ (spike at $n$ is due to the spike position being chosen as $\xi_{i}$ before the shift) or if $(i-1) M+\xi_{i-1}+m_{0}=n$ (spike from a previous interval has been shifted to the right into the interval under consideration). These two possibilities are mutually exclusive. Hence, we must have either $\xi_{i}+m_{0}=u$ or $\xi_{i-1}+m_{0}=u+M$. That these events are mutually exclusive follows from subtracting them to yield $\xi_{i}=\xi_{i-1}-M$, which is not possible. Thus, we have that

$$
\begin{aligned}
P[v[n]=1]=P\left[\xi_{i}=u-m_{0}\right]+ & P\left[\xi_{i-1}=u+M-m_{0}\right] \\
=\sum_{k=0}^{M-1} P\left[\xi_{i}\right. & \left.=u-k \mid m_{0}=k\right] P\left[m_{0}=k\right] \\
& +\sum_{k=0}^{M-1} P\left[\xi_{i-1}=u+M-k \mid m_{0}=k\right] P\left[m_{0}=k\right] .
\end{aligned}
$$




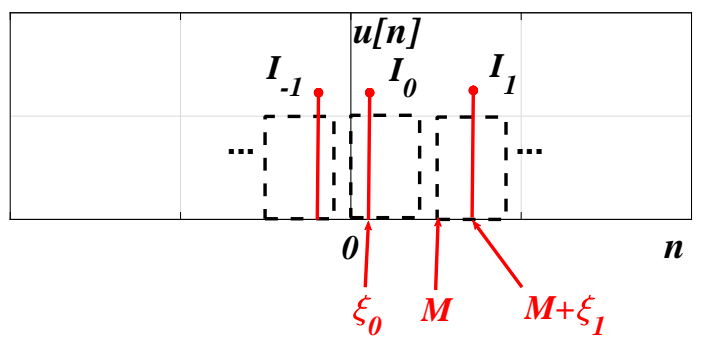

Figure 11: Spike process realization before applying the shift $m_{0}$.

But $\xi_{i}$ and $\xi_{i-1}$ are both independent of $m_{0}$ so that we have

$$
\begin{aligned}
P[v[n]=1]= & \sum_{k=0}^{M-1} P\left[\xi_{i}=u-k\right] P\left[m_{0}=k\right] \\
& +\sum_{k=0}^{M-1} P\left[\xi_{i-1}=u+M-k\right] P\left[m_{0}=k\right] \\
= & \sum_{k=0}^{u} P\left[\xi_{i}=u-k\right] P\left[m_{0}=k\right] \\
& +\sum_{k=u+1}^{M-1} P\left[\xi_{i-1}=u+M-k\right] P\left[m_{0}=k\right] \\
= & \sum_{k=0}^{u} P\left[\xi_{i}=u-k\right] \frac{1}{M} \\
& +\sum_{k=u+1}^{M-1} P\left[\xi_{i-1}=u+M-k\right] \frac{1}{M} \\
= & \frac{1}{M}\left(\sum_{l=0}^{u} P\left[\xi_{i}=l\right]+\sum_{l=u+1}^{M-1} P\left[\xi_{i-1}=l\right]\right) \\
= & \frac{1}{M}\left(\sum_{l=0}^{u} \frac{1}{M}+\sum_{l=u+1}^{M-1} \frac{1}{M}\right) \\
= & \frac{1}{M} .
\end{aligned}
$$

Therefore, $E[v[n]]=1 / M$.

\section{Second-order PMF}

Since $v[n]=1$ or $v[n]=0$ the second-order moment is $E\left[v\left[n_{1}\right] v\left[n_{2}\right]\right]=$ $P\left[v\left[n_{1}\right]=1, v\left[n_{2}\right]=1\right]$. Letting $n_{2}=n_{1}+\Delta$ for $\Delta \geq 0$ we next determine 


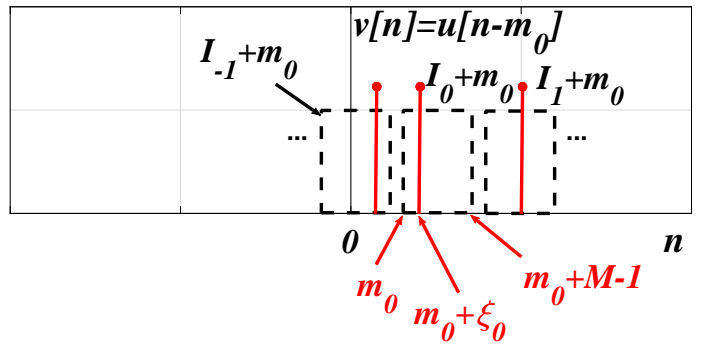

Figure 12: Spike process realization.

$P\left[v\left[n_{1}\right]=1, v\left[n_{2}\right]=1\right]$ for the three cases of $\Delta$.

First case: $\Delta=0$

Here $n_{1}=n_{2}$ and therefore

$$
P\left[v\left[n_{1}\right]=1, v\left[n_{2}\right]=1\right]=P\left[v\left[n_{1}\right]=1\right]=\frac{1}{M} .
$$

Second case: $\Delta \geq M$

In this case $n_{1}$ and $n_{2}$ must be in different intervals as shown by the dashed boxes in Fig. 11. As a result, the spike events must be conditionally independent, i.e., for a given $m_{0}$, the spikes within the dashed boxes are independent by Assumption 1. Therefore,

$$
\begin{gathered}
P\left[v\left[n_{1}\right]=1, v\left[n_{2}\right]=1\right]= \\
P\left[u\left[n_{1}-m_{0}\right]=1, u\left[n_{2}-m_{0}\right]=1\right] \\
=\sum_{k=0}^{M-1} P\left[u\left[n_{1}-k\right]=1, u\left[n_{2}-k\right]=1 \mid m_{0}=k\right] P\left[m_{0}=k\right] \\
=\sum_{k=0}^{M-1} P\left[u\left[n_{1}-k\right]=1, u\left[n_{2}-k\right]=1\right] P\left[m_{0}=k\right] \\
=\sum_{k=0}^{M-1} P\left[u\left[n_{1}-k\right]=1\right] P\left[u\left[n_{2}-k\right]=1\right] P\left[m_{0}=k\right] \\
=\sum_{k=0}^{M-1} \frac{1}{M^{2}} \frac{1}{M}=\frac{1}{M^{2}} .
\end{gathered}
$$




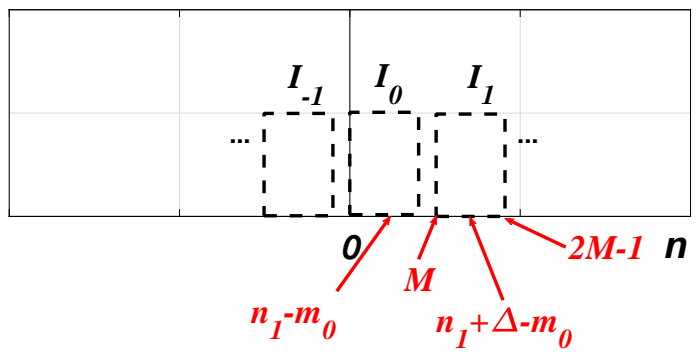

Figure 13: Case 3a.

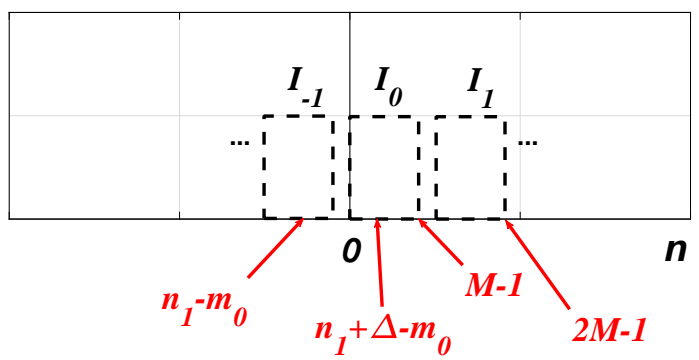

Figure 14: Case 3b.

Third case: $1 \leq \Delta \leq M-1$

Assume without loss of generality that the points under consideration for $v[n]$ are at $n_{1} \in I_{0}$ and $n_{1}+\Delta \in I_{1}$. For a nonzero probability the spikes in $u[n]$, i.e., prior to shifting by $m_{0}$, must be in two adjacent intervals, $I_{i-1}$ and $I_{i}$. This is because $\Delta \leq M-1$. Alternatively, we must have that $n_{1}-m_{0} \in I_{i-1}$ and $n_{1}+\Delta-m_{0} \in I_{i}$. One possibility is shown in Fig. 13 for $i=1$. Hence, for spikes in $v[n]$ at $n_{1} \in I_{0}$ and $n_{1}+\Delta \in I_{1}$ we must have $n_{1}-m_{0} \in I_{0}$ and $n_{1}+\Delta-m_{0} \in I_{1}$ or equivalently, $0 \leq n_{1}-m_{0} \leq M-1$ and $M \leq n_{1}+\Delta-m_{0} \leq 2 M-1$. This is deemed Case 3a. But there is a second possibility which is possible as shown in Fig. 14. This is called Case $3 \mathrm{~b}$ and occurs when the unshifted spikes of $u[n]$ are in the adjacent intervals $I_{-1}$ and $I_{0}$. Which case occurs depends upon the amount of the shift $m_{0}$ as well as $\Delta$. Consider first Case 3a. Then we must have $n_{1}-m_{0} \in I_{0}$ and 
$n_{1}+\Delta-m_{0} \in I_{1}$ and therefore

$$
\begin{aligned}
& 0 \leq n_{1}-m_{0} \leq M-1 \\
& M \leq n_{1}+\Delta-m_{0} \leq 2 M-1
\end{aligned}
$$

or

$$
\begin{aligned}
& -(M-1) \leq m_{0}-n_{1} \leq 0 \\
& -(2 M-1) \leq m_{0}-\left(n_{1}+\Delta\right) \leq-M
\end{aligned}
$$

or

$$
\begin{aligned}
& n_{1}-(M-1) \leq m_{0} \leq n_{1} \\
& n_{1}-(M-1)+(\Delta-M) \leq m_{0} \leq n_{1}+(\Delta-M) .
\end{aligned}
$$

But $(\Delta-M)<0$ so that the larger of the two lower limits is $n_{1}-(M-1)$ and the smaller of the two upper limits is $n_{1}+(\Delta-M)$. As a result, we have that $n_{1}-(M-1) \leq m_{0} \leq n_{1}+(\Delta-M)$. Also, since it was assumed that $n_{1} \in I_{0}$, $n_{1}-(M-1) \leq 0$, which becomes the lower limit, and hence

$$
0 \leq m_{0} \leq n_{1}+\Delta-M
$$

The upper value is $\geq 0$ since $n_{1}+\Delta \geq M$ but also $\leq M-1$ since $n_{1}+\Delta \leq 2 M-1$. Hence, all possible values of $m_{0}$ determined by the inequality have probability of $1 / M$. There are a total of $n_{1}+\Delta-M+1$ terms. Next consider Case 3b, which proceeds in a similar manner. Then we must have $n_{1}-m_{0} \in I_{-1}$ and $n_{1}+\Delta-m_{0} \in I_{0}$ and therefore

$$
\begin{aligned}
& -M \leq n_{1}-m_{0} \leq-1 \\
& 0 \leq n_{1}+\Delta-m_{0} \leq M-1
\end{aligned}
$$




$$
\begin{aligned}
& 1 \leq m_{0}-n_{1} \leq M \\
& -(M-1) \leq m_{0}-\left(n_{1}+\Delta\right) \leq 0
\end{aligned}
$$

or

$$
\begin{aligned}
& n_{1}+1 \leq m_{0} \leq n_{1}+M \\
& \left(n_{1}+\Delta\right)-(M-1) \leq m_{0} \leq n_{1}+\Delta .
\end{aligned}
$$

But $1 \leq \Delta \leq M-1$ so that the upper limit must be $n_{1}+\Delta$. Also, $\left(n_{1}+\Delta\right)-$ $(M-1)=n_{1}+1+(\Delta-M) \leq n_{1}+1$ so that the lower limit becomes $n_{1}+1$. We thus have

$$
n_{1}+1 \leq m_{0} \leq n_{1}+\Delta
$$

But it was assumed that $n_{1}+\Delta \geq M$ and therefore the upper limit must be $M-1$ for a nonzero probability of $m_{0}$. Also, note that $n_{1} \neq 0$ since $\Delta$ would need to be $M$ for $n_{1}+\Delta \in I_{1}$. Therefore, as a result, we have finally that $n_{1}+1 \leq m_{0} \leq M-1$, and the number of terms is $M-1-\left(n_{1}+1\right)+1=M-1-n_{1}$. Combining this with the number of terms for Case 3a, which is $n_{1}+\Delta-M+1$, yields a total number of terms of $\Delta$. 
Now we have

$$
\begin{gathered}
P\left[v\left[n_{1}\right]=1, v\left[n_{2}\right]=1\right]=P\left[u\left[n_{1}-m_{0}\right]=1, u\left[n_{2}-m_{0}\right]=1\right] \\
=\sum_{k} P\left[u\left[n_{1}-k\right]=1, u\left[n_{2}-k\right]=1 \mid m_{0}=k\right] P\left[m_{0}=k\right] \\
=\sum_{k} P\left[u\left[n_{1}-k\right]=1, u\left[n_{2}-k\right]=1\right] P\left[m_{0}=k\right] \\
=\sum_{k} P\left[u\left[n_{1}-k\right]=1\right] P\left[u\left[n_{2}-k\right]=1\right] P\left[m_{0}=k\right] \\
=\sum_{k} \frac{1}{M^{2}} \frac{1}{M}=\frac{\Delta}{M^{3}}
\end{gathered}
$$

In summary, we have for the second-order PMF

$$
P\left[v\left[n_{1}\right]=1, v\left[n_{1}+\Delta\right]=1\right]= \begin{cases}\frac{1}{M} \quad \Delta=0 \\ \frac{\Delta}{M^{3}} \quad 1 \leq \Delta \leq M-1 \\ \frac{1}{M^{2}} \quad \Delta \geq M\end{cases}
$$

Note also that it is only dependent on the spacing between samples, and hence, this will produce a WSS random process for $v[n]$.

\section{Moments of $v[n]$}

As already shown $E[v[n]]=1 / M$ for all $n$. To compute the ACS we have $r_{v}[k]=E[v[n] v[n+k]]=1 \cdot P[v[n]=1, v[n+k]=1]$, and from the above result with $k$ replacing with $\Delta$, and since $c_{v}[k]=r_{v}[k]-E^{2}[v[n]]$ then

$$
c_{v}[k]= \begin{cases}\frac{1}{M}\left(1-\frac{1}{M}\right) & k=0 \\ -\frac{1}{M^{2}}\left(1-\frac{k}{M}\right) & \\ 0 & k \geq M\end{cases}
$$


Hence, the ACS and PSD for the process $h[n]=a[n] v^{\prime}[n]$, where $v^{\prime}[n]=v[n]-\mu_{v}$, and $\mu_{v}=\frac{1}{M}$, are

$$
r_{h}[k]=\left\{\begin{array}{cc}
\frac{1}{M^{2}}(M-1) \sigma_{A}^{2} & k=0 \\
0 & 1 \leq k \leq M-1 \\
0 & k \geq M
\end{array}\right.
$$

and

$$
P_{h}(f)=\frac{1}{M^{2}}(M-1) \sigma_{A}^{2}
$$

where $\frac{-1}{2} \leq f \leq \frac{1}{2}$ 


\section{Appendix 1B. Probabilistically Motivated Model as a Filtered Poisson Process (not included in the paper)}

In this appendix (which is not part of the paper) we will present a new way of modeling our problem using a filtered Poisson process. We formulate the problem as following: we transmit a known ultrasonic signal through the material and we wait to receive the reflected signal to decide if a defect exists or not. The physically motivated model proposed in the paper presented the interaction between the material and the transmitted signal as a simple model which shift and change the amplitude of the transmitted signal. In the same way we can model our material as a filtered Poisson process as following (we will present everything in the continuous time)

$$
x(t)=\sum_{i=-\infty}^{\infty} A_{i} \delta\left(t-\tau_{i}\right)
$$

where $x(t)$ is called Poisson impulse train, and the number of impulses $N(t)$ occurring in any interval $I$ of duration $T$ is given by

$$
P[N(T)=k]=\frac{(\lambda T)^{k}}{k !} e^{-(\lambda T)}
$$

where $\lambda$ is the average rate per unit of time, which can be measured in practice. $A_{i}$ 's are IID random variables independent of $\tau_{i}$ and $N(t)$. The Poisson process has a lot of attractive properties such as that the increments of non-overlapping interval (disjoint) are statistically independent and many more.

\section{Statistical Properties}

To get the first-order PMF of our new model we will start with the filtered process $y(t)$ which is the output of following system

$$
x(t)=\sum_{i=-\infty}^{\infty} A_{i} h\left(t-\tau_{i}\right)
$$

and at a particular instant of time $t$ we have

$$
y(t)=\sum_{i=-\infty}^{\infty} A_{i} h\left(t-\tau_{i}\right)=\frac{N_{\Delta}}{\Delta}
$$




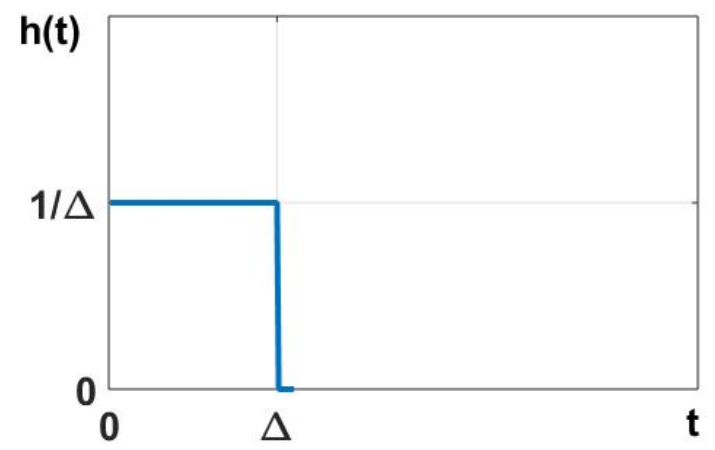

Figure 15: Linear Time Invariant Filter Impulse Response

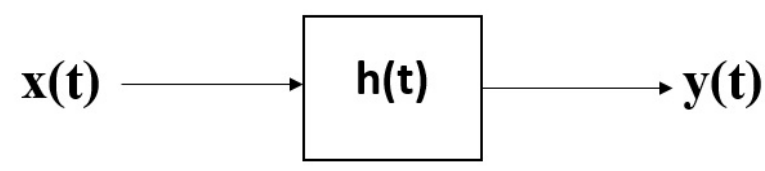

Figure 16: Linear Time Invariant System

where $N_{\Delta}$ indicates the number of impulses occurring in the interval $[t-\Delta, t]$ Noticing that the process $y(t)$ tends to the process $x(t)$ as $\Delta \rightarrow 0$, with this in mind we start to get the first order-PMF statistics for our model. First we start with $E[y(t)]$ :

$$
\begin{aligned}
E[y(t)] & =E\left[\sum_{i=-\infty}^{\infty} A_{i} h\left(t-\tau_{i}\right)\right]=\sum_{i}^{N_{\Delta}} E\left[A_{i}\right] E\left[h\left(t-\tau_{i}\right)\right] \\
& =\mu_{A} \sum_{i}^{N_{\Delta}} E\left[h\left(t-\tau_{i}\right)\right] \\
& =\frac{\mu_{A}}{\Delta} E\left[N_{\Delta}\right] \\
& =\frac{\mu_{A}}{\Delta}(\Delta \lambda)=\lambda \mu_{A}
\end{aligned}
$$


Now for $E\left[y\left(t_{1}\right) y\left(t_{2}\right)\right]$ without loss of generality assume $t_{2}>t_{1}$ for this case we have

$$
\begin{gathered}
E\left[y\left(t_{1}\right) y\left(t_{2}\right)\right]=E\left[\sum_{i=-\infty}^{\infty} \sum_{j=-\infty}^{\infty} A_{i} A_{j} h\left(t_{1}-\tau_{i}\right) h\left(t_{2}-\tau_{j}\right)\right] \\
E\left[y\left(t_{1}\right) y\left(t_{2}\right)\right]=E\left[\sum_{i=-\infty}^{\infty} A_{i}^{2} h\left(t_{1}-\tau_{i}\right) h\left(t_{2}-\tau_{i}\right)+\sum_{i=-\infty}^{\infty} \sum_{\substack{j=-\infty \\
i \neq j}}^{\infty} A_{i} A_{j} h\left(t_{1}-\tau_{i}\right) h\left(t_{2}-\tau_{j}\right)\right] \\
E\left[y\left(t_{1}\right) y\left(t_{2}\right)\right]=E\left[\sum_{i=-\infty}^{\infty} A_{i}^{2} h\left(t_{1}-\tau_{i}\right) h\left(t_{2}-\tau_{i}\right)\right]+E\left[\sum_{i=-\infty}^{\infty} \sum_{\substack{j=-\infty \\
i \neq j}}^{\infty} A_{i} A_{j} h\left(t_{1}-\tau_{i}\right) h\left(t_{2}-\tau_{j}\right)\right] \\
E\left[y\left(t_{1}\right) y\left(t_{2}\right)\right]=E\left[A^{2}\right] E\left[\sum_{i=-\infty}^{\infty} h\left(t_{1}-\tau_{i}\right) h\left(t_{2}-\tau_{i}\right)\right]+\mu_{A}^{2} E\left[\sum_{\substack{i=-\infty \\
j}}^{\infty} \sum_{\substack{j=-\infty \\
i \neq j}}^{\infty} h\left(t_{1}-\tau_{i}\right) h\left(t_{2}-\tau_{j}\right)\right]
\end{gathered}
$$

we will take the first term first $E\left[\sum_{i=-\infty}^{\infty} h\left(t_{1}-\tau_{i}\right) h\left(t_{2}-\tau_{i}\right)\right]$ This term will only be nonzero if

$$
\left|t_{2}-t_{1}\right| \leq \Delta
$$

which means that the two boxes are overlapping otherwise the results will be zero. call

$$
t_{2}-t_{1}=\tau
$$

and the box width for which this is nonzero is $\Delta-|\tau|$. so we have

$$
\begin{gathered}
E\left[\sum_{i=-\infty}^{\infty} h\left(t_{1}-\tau_{i}\right) h\left(t_{2}-\tau_{i}\right)\right]=\sum_{i=-\infty}^{\infty} E\left[h\left(t_{1}-\tau_{i}\right) h\left(t_{2}-\tau_{i}\right)\right] \\
\sum_{i=-\infty}^{\infty} E\left[h\left(t_{1}-\tau_{i}\right) h\left(t_{2}-\tau_{i}\right)\right]=\left(\frac{\lambda(\Delta-|\tau|)}{\Delta^{2}}\right) \\
E\left[\left(\sigma_{A}^{2}+\mu_{A}^{2}\right) \sum_{i=-\infty}^{\infty} h\left(t_{1}-\tau_{i}\right) h\left(t_{2}-\tau_{i}\right)\right]=\left\{\begin{array}{cc}
\left(\sigma_{A}^{2}+\mu_{A}^{2}\right)\left(\frac{\lambda(\Delta-|\tau|)}{\Delta^{2}}\right) & |\tau| \leq \Delta \\
0 & |\tau|>\Delta
\end{array}\right.
\end{gathered}
$$


Now let us take the second term $E\left[\sum_{i=-\infty}^{\infty} \sum_{\substack{j=-\infty \\ i \neq j}}^{\infty} h\left(t_{1}-\tau_{i}\right) h\left(t_{2}-\tau_{j}\right)\right]$ notice that

$$
\begin{aligned}
E\left[\sum_{i=-\infty}^{\infty} \sum_{\substack{j=-\infty \\
i \neq j}}^{\infty} h\left(t_{1}-\tau_{i}\right) h\left(t_{2}-\tau_{j}\right)\right]= \\
E\left[\sum_{i=-\infty}^{\infty} \sum_{j=-\infty}^{\infty} h\left(t_{1}-\tau_{i}\right) h\left(t_{2}-\tau_{j}\right)\right]-E\left[\sum_{i=-\infty}^{\infty} h\left(t_{1}-\tau_{i}\right) h\left(t_{2}-\tau_{i}\right)\right]
\end{aligned}
$$

As we can see the second term is exactly like the one we solved above, however, the first term is not. Now for

$$
E\left[\sum_{i=-\infty}^{\infty} \sum_{j=-\infty}^{\infty} h\left(t_{1}-\tau_{i}\right) h\left(t_{2}-\tau_{j}\right)\right]
$$

same thing we have two cases first start with $|\tau|>\Delta$ that means the two boxes are not overlapping we get

$$
E\left[\sum_{i=-\infty}^{\infty} \sum_{j=-\infty}^{\infty} h\left(t_{1}-\tau_{i}\right) h\left(t_{2}-\tau_{j}\right)\right]=\sum_{i=-\infty}^{\infty} \sum_{j=-\infty}^{\infty} E\left[h\left(t_{1}-\tau_{i}\right) h\left(t_{2}-\tau_{j}\right)\right]
$$

since there are double summation then both boxes contribute to the sum and since each box has a width of $\Delta$ we get

$$
\sum_{i=-\infty}^{\infty} \sum_{j=-\infty}^{\infty} E\left[h\left(t_{1}-\tau_{i}\right) h\left(t_{2}-\tau_{j}\right)\right]=\left(\frac{(\lambda \Delta)^{2}}{\Delta^{2}}\right)
$$

next we do the case when the two boxes overlap that is $|\tau| \leq \Delta$

$$
\sum_{i=-\infty}^{\infty} \sum_{j=-\infty}^{\infty} E\left[h\left(t_{1}-\tau_{i}\right) h\left(t_{2}-\tau_{j}\right)\right]=\frac{1}{\Delta^{2}} E\left[\left(N_{I_{1}}+N_{I_{2}}\right)\left(N_{I_{2}}+N_{I_{3}}\right)\right]
$$

in this case all intervals will contribute in the sum interval one which is box one with no overlapping and then interval two which is the nonoverlapping for box number two and the shared interval between the two boxes call the number of impulses in the three intervals as following: $N_{I_{1}}, N_{I_{2}}$, and $N_{I_{3}}$, we get

$$
\sum_{i=-\infty}^{\infty} \sum_{j=-\infty}^{\infty} E\left[h\left(t_{1}-\tau_{i}\right) h\left(t_{2}-\tau_{j}\right)\right]=\frac{1}{\Delta^{2}} E\left[\left(N_{I_{1}} N_{I_{2}}+N_{I_{1}} N_{I_{3}}+N_{I_{2}}^{2}+N_{I_{2}} N_{I_{3}}\right)\right]
$$


and as we stated the number of impulses in any disjoint interval are statistically independent we get

$$
\sum_{i=-\infty}^{\infty} \sum_{j=-\infty}^{\infty} E\left[h\left(t_{1}-\tau_{i}\right) h\left(t_{2}-\tau_{j}\right)\right]=\left[\lambda^{2}+\frac{\lambda}{\Delta}\left(1-\frac{|\tau|}{\Delta}\right)\right]
$$

put it all together

$$
R_{y}(\tau)=E\left[y\left(t_{1}\right) y\left(t_{2}\right)\right]=\left\{\begin{array}{cl}
{\left[\mu_{A}^{2} \lambda^{2}+\left(\sigma_{A}^{2}+\mu_{A}^{2}\right) \frac{\lambda}{\Delta}\left(1-\frac{|\tau|}{\Delta}\right)\right]} & |\tau| \leq \Delta \\
\mu_{A}^{2} \lambda^{2} & |\tau|>\Delta
\end{array}\right.
$$

to get the power spectral density we take the Fourier transform of the ACS to get

$$
P_{y}(f)=\mu_{A}^{2} \lambda^{2} \delta(f)+\left(\sigma_{A}^{2}+\mu_{A}^{2}\right) \lambda \operatorname{sinc}(\Delta f)
$$

Now let $\Delta \rightarrow 0$ to get the PSD of the process $x(t)$ we get

$$
P_{x}(f)=\lim _{\Delta \rightarrow \infty} P_{y}(f)=\mu_{A}^{2} \lambda^{2} \delta(f)+\left(\sigma_{A}^{2}+\mu_{A}^{2}\right) \lambda
$$

Now for any filter $h(t)$ we get that the output $y(t)$ PSD is

$$
P_{y}(f)=\left(\sigma_{A}^{2}+\mu_{A}^{2}\right) \lambda|H(f)|^{2}+\left(\mu_{A} \lambda H(0)\right)^{2} \delta(f)
$$

if $A$ 's have zero mean we get

$$
P_{y}(f)=\sigma_{A}^{2} \lambda|H(f)|^{2}
$$

Which interesting enough matches the result we got from our physically motivated model, where the clutter has a flat spectrum hat is a scaled version of the ESD of the transmitted signal.

\section{List of References}

[1] J. P. Charlesworth and J. A. G. Temple, Engineering applications of ultrasonic time-of-flight diffraction. Vol. 1. John Wiley \& Sons Inc, 1989. 
[2] J. Blitz and G. Simpson, Ultrasonic methods of non-destructive testing. Vol. 2. Springer Science \& Business Media, 1995.

[3] S. Kay, "Optimal signal design for detection of gaussian point targets in stationary gaussian clutter/reverberation," IEEE journal of selected topics in signal processing 1.1, vol. 31, pp. 31-41, 2007.

[4] M. R. Bell, "Information theory and radar waveform design," IEEE Transactions on Information Theory, vol. 39, no. 5, pp. 1578-1597, 1993.

[5] S. U. Pillai, D. Youla, H. Oh, and J. R. Guerci, "Optimum transmit-receiver design in the presence of signal-dependent interference and channel noise," in Conference Record of the Thirty-Third Asilomar Conference on Signals, Systems, and Computers (Cat. No. CH37020), vol. 2. IEEE, 1999, pp. 870875.

[6] J. Krautkrämer and H. Krautkrämer, "Ultrasonic testing by determination of material properties," in Ultrasonic Testing of Materials. Springer, 1990.

[7] P. Ramuhalli, A. D. Cinson, S. L. Crawford, R. V. Harris, A. A. Diaz, and M. T. Anderson, "Evaluation of signal processing tools for improving phased array ultrasonic weld inspection," in Smart Sensor Phenomena, Technology, Networks, and Systems 2011, vol. 7982. International Society for Optics and Photonics, 2011.

[8] P. Ramuhalli, M. S. Good, A. A. Diaz, M. T. Anderson, B. E. Watson, T. J. Peters, M. Dixit, and L. J. Bond, "Ultrasonic characterization of cast austenitic stainless steel microstructure: Discrimination between equiaxedand columnar-grain material-an interim study," Pacific Northwest National Lab.(PNNL), Richland, WA (United States), Tech. Rep., 2009.

[9] K.-J. Langenberg, R. Marklein, and K. Mayer, Ultrasonic nondestructive testing of materials: theoretical foundations. CRC Press, 2012.

[10] M. G. Gustafsson, "Nonlinear clutter suppression using split spectrum processing and optimal detection," IEEE transactions on ultrasonics, ferroelectrics, and frequency control, vol. 43, pp. 109-124, 1996.

[11] M. G. Gustafsson and T. Stepinski, "Studies of split spectrum processing, optimal detection, and maximum likelihood amplitude estimation using a simple clutter model," Ultrasonics, vol. 35, pp. 31-52, 1997.

[12] F. S. Cohen, "Modeling of ultrasound speckle with application in flaw detection in metals," IEEE Transactions on signal processing, vol. 40, pp. 624-632, 1992. 
[13] C.-P. Chiou, R. B. Thompson, and L. W. Schmerr, "Model-based signal processing techniques for ultrasonic flaw detection: Simulation studies," in Review of Progress in Quantitative Nondestructive Evaluation. Springer, 1993, pp. 703-710.

[14] I. Yalda-Mooshabad, F. J. Margetan, and R. B. Thompson, "Monte-carlo simulation of ultrasonic grain noise," in Review of progress in Quantitative Nondestructive Evaluation. Springer, 1993, pp. 1727-1734.

[15] S. Chatillon, C. Poidevin, N. Gengembre, and A. Lhemery, "Simplified modeling of backscattered noise and attenuation phenomena for quantitative performance demonstration of ut methods," in AIP Conference Proceedings. IOP INSTITUTE OF PHYSICS PUBLISHING LTD, 2003, pp. 93-100.

[16] V. DORVAL, L. DUCOUSSO-GANJEHI, S. CHATILLON, and F. JENSON, "Modeling ultrasonic structural noise based on the microstructural properties of metals in the context of non destructive evaluation," in 13th International Symposium on Nondestructive Characterization of Materials (NDCM-XIII), 2013, pp. 20-24.

[17] K. D. Donohue, "Maximum likelihood estimation of a-scan amplitudes for coherent targets in media of unresolvable scatterers," IEEE transactions on ultrasonics, ferroelectrics, and frequency control, vol. 39.3, pp. 422-431, 1992.

[18] J. Saniie, T. Wang, and N. M. Bilgutay, "Statistical evaluation of backscattered ultrasonic grain signals," The Journal of the Acoustical Society of America, vol. 84, pp. 400-408, 1988.

[19] S. Kay, Intuitive probability and random processes using MATLAB囚. Springer Science \& Business Media, 2006.

[20] I. M. Jacobs and J. Wozencraft, "Principles of communication engineering." 1965.

[21] H. L. Van Trees and K. L. Bell, Detection estimation and modulation theory. John Wiley \& Sons, 2004.

[22] R. F. Wagner, "Statistics of speckle in ultrasound b-scans," IEEE Trans. Sonics \& Ultrason., vol. 30, pp. 156-163, 1983.

[23] T. F. Quatieri, Discrete-time speech signal processing: principles and practice. Pearson Education India, 2006.

[24] T. Dreyer, W. Krauss, E. Bauer, and R. Riedlinger, "Investigations of compact self focusing transducers using stacked piezoelectric elements for strong sound pulses in therapy," in 2000 IEEE Ultrasonics Symposium. Proceedings. An International Symposium (Cat. No. 00CH37121), vol. 2. IEEE, 2000, pp. 1239-1242. 
[25] S. M. Kay, Fundamentals of Statistical Signal Processing: Detection. Prentice Hall PTR, 1998.

[26] S. M. Kay, Fundamentals of Statistical Signal Processing, Volume I: Estimation Theory. Prentice Hall PTR, 1993.

[27] S. Nadarajah and S. Kotz, "Exact distribution of the max/min of two gaussian random variables," IEEE Transactions on very large scale integration (VLSI) systems, vol. 16, no. 2, pp. 210-212, 2008. 


\title{
CHAPTER 2
}

\section{Optimal Signal Design For a New Physically Motivated Clutter Model With Applications to Ultrasonic Testing}

\author{
by \\ Yazan Rawashdeh and Steven Kay \\ Dept. of Electrical, Computer and Biomedical Engineering \\ University of Rhode Island, Kingston, RI, USA
}

Under review in IEEE Transactions on Ultrasonics, Ferroelectrics, and Frequency Control, 2020. 


\section{Abstract}

This paper is a continuation of our previous work for a new physically motivated clutter model, where we explore the optimal signal design problem. The reason to pursue the optimal signal design is that the model, even though it was simple, led to a robust model that worked well on real ultrasonic data. Moreover, by using the model an analytical solution for the optimal signal is obtained. In addition, the model gives new insight into the signal design problem that can be valuable for many applications. A new proof has been provided for a finite data record that is lacking in the literature. We have found that the optimal signal is an impulse and as a result the signal has an impulsive autocorrelation sequence (ACS). Since an impulsive ACS signal is not realizable in practice a study for an alternative signal is conducted. Signals from four different categories have been explored: linear frequency modulated (LFM) signal, non-linear frequency modulated (NLFM) signals, phase coded modulated (PCM) signals, and finally what we called other signals. A comparison analysis between these signals themselves and the most commonly used excitation signal in practice, which is the Gaussian amplitude modulated sinusoid (GAMS) signal, has been implemented. We show that the LFM signal has a large advantage over the GAMS signal in terms of detectability. In addition, a comparison between the LFM signal and the GAMS signal under a deviation from the single scatterer assumption, indicating a more complex target, using simulated noise is performed.

\subsection{Introduction}

The optimal signal design problem in signal-dependent noise has been of interest to researchers for a long time. Signal-dependent noise is referred to as clutter in radar, backscattering noise in ultrasonic non-destructive testing (NDT)

of materials, and reverberation in sonar. However, the term clutter has been used 
interchangeably with backscattering noise, and we will follow this convention here. The optimal signal design in the presence of signal-dependent noise is of interest to many fields such as: radar, sonar, and NDT [1],[2],[3]. The goal in a NDT session is to be able to detect defects inside the material. However, the presence of clutter hinders this task. The fact that the characteristics of the clutter are signal dependent complicates the optimal signal design problem. In terms of noise we can categorize the statistical problem into two categories. First, where the noise is not signal dependent and for this case the solution for the optimal signal design is well known. Whether the noise in this case is white or colored, as long as it is not dependent on the transmitted signal, it says to put all the signal energy into the frequency band where the noise power is minimum. Moreover, this implies that for a discrete signal vector one should choose the signal as the eigenvector of the noise covariance matrix whose eigenvalue is minimum [4, p.110]. The second case, where the noise is signal dependent, and which is the case in NDT applications, there has been no analytical solution. The signal design varies corresponding to how the problem is framed statistically and the type of model assumed, which is a subjective matter and not an objective one.

In this paper, we continue our work that was done in [5], in an effort to create a unified framework to solve the problem of detecting defects in material in a NDT session. In [5] we presented an efficient and simple model for the clutter noise. Even though the model of the return signals did not include the frequency-dependent attenuation and distant-dependent attenuation, it led to many excellent results:

1. The robust model works well on real ultrasonic data.

2. An analytical solution for the optimal signal design is obtained.

3. Flexibility to be extended to more complicated cases such as the case of 2-Dimensional data, which can be used in medical imaging applications. 
4. A new insight on the signal design problem that can fit many applications depending on the problem at hand.

Therefore, a proof is provided for the optimal signal in the presence of a signaldependent noise with a finite data record that is lacking in the literature. We find that the optimal signal is an impulse and as a result the signal has an impulsive autocorrelation sequence (ACS). All proofs that are available in literature usually convert the problem to the frequency domain, which assumes that the data record is large. In comparison, in this paper we provide a proof for a finite data record and we utilize the data in the time/space domain. Since an impulsive signal is not realizable in practice a study for an alternative signal is conducted. Signals from four different categories have been explored: linear frequency modulated (LFM) signal, non-linear frequency modulated (NLFM) signals, phase coded modulated (PCM) signals. Finally, we introduce two other signals that are based on minimizing the integrated sidelobe level (ISL) function, which are grouped into what we call the "other signals". Conventionally LFM, NLFM, and PCM signals are called compressed signals. In the context of NDT of materials the use of compressed signals: LFM signal, NLFM signal, and PCM signal, to improve the performance in terms of detectability, enhancing the range resolution, and improving the signal to noise ratio (SNR), has been applied for a range of applications [3],[6],[7],[8],[9],[10]. In [3] the authors introduced a new window function, based on the reactance transform, in combination with a compressed signal where they used an LFM signal and an NLFM signal and showed that the approach is valid for NDT techniques that use broadband excitations such as eddy current and thermography. In [7] the authors used an experimental approach, since the NLFM signal can be tailored to reproduce any desired continuous spectrum, to adapt the excitation signal to the probes. Then they proceeded to apply the approach on air-coupled ultrasound imaging. 
In [8] the authors produced pulse compressed techniques such as eddy current and thermography in an actual lab setup and implemented the analysis experimentally. In [9] the authors used a random frequency modulated (FM) excitation, which still falls into the compressed signals category. Instead of an LFM signal to improve the performance of multiple input multiple output system. However, in [3], [6]-[10] the statistical framework was lacking. In comparison, in this work we create a unified framework, where the problem is analyzed statistically. We provide a model for the clutter noise, and produce an analytical solution for the optimal signal to be used in combination with the model. Also, a comparison between the suggested signals and the most commonly used excitation signal in practice, which is the Gaussian amplitude modulated sinusoid (GAMS) signal, was performed.

In this paper, we first state the detection problem and provide a model review in section 2.2, followed by a performance derivation of the generalized matched filter (GMF) in section 2.3. Next, in section 2.4 we maximize the deflection coefficient with respect to the transmitted signal. In section 2.5 we explore different signals with good ACS properties. In section 2.6 we perform a comparison analysis based on the clutter to noise ratio $(\mathrm{CNR})$ verses the deflection coefficient, also using the receiver operating characteristics (ROC) curve. Moreover, a simulation comparison between the GAMS signal verses the LFM signal was conducted. Finally, conclusions are given in section 2.7.

\subsection{Problem Statement and Review of the Model}

The model for the received signal shown in Fig. 17.

$$
\begin{aligned}
& \mathcal{H}_{0}: x[n]=c[n]+w[n] \\
& \mathcal{H}_{1}: x[n]=A_{0} s\left[n-n_{0}\right]+c[n]+w[n]
\end{aligned}
$$


To formulate the problem in a statistical framework, similar to what was done in [5], we assume that the received signal is $x[n]=c[n]+w[n]$ under hypothesis $\mathcal{H}_{0}$, where $c[n]$ denotes the clutter noise, and $w[n]$ denotes the ambient white noise. Under the hypothesis $\mathcal{H}_{1}$, we get $x[n]=A_{0} s\left[n-n_{0}\right]+c[n]+w[n]$, where the defect impulse response $g[n]$ is modeled as a point target (a shift and scaling of the transmitted signal $s[n])$. Even though this assumption does not account for the distance-dependent attenuation and frequency-dependent attenuation, our model in [5] showed excellent results on real ultrasonic data. The amplitude $A_{0}$ and delay $n_{0}$ represent the scale factor and delay that correspond to the target geometry and location, respectively. The transmitted ultrasonic pulse $s[n]$ is specified by the type of transducer that is used for testing. In this paper, we will examine the optimal signal design $s[n]$ that is optimal in terms of detectability. We then model the clutter $c[n]$ as the output of a random linear time-invariant (LTI) filter with the impulse response $h[n]$, when the input is the transmitted ultrasonic pulse $s[n]$.

As shown in Fig. 17 there are two types of noise that will affect our received signal. The first is ambient white noise $w[n]$, which is assumed to be an independent identically distributed (IID) with a probability density function (PDF) $\mathbf{w} \sim \mathcal{N}\left(\mathbf{0}, \sigma_{w}^{2} \mathbf{I}\right)$ (white Gaussian noise process). The boldface $\mathbf{w}$ indicates the vector of ambient white noise samples, and the symbol " " means distributed as. The ambient white noise can be reduced by simple averaging of multiple A-scans for the same scanned volume. Second, we have the clutter noise $c[n]$ that is caused by the material microstructure, and is assumed to be a colored Gaussian noise process with a $\mathrm{PDF} \mathbf{c} \sim \mathcal{N}\left(\mathbf{0}, \mathbf{C}_{c}\right)$, where $\mathbf{C}_{c}$ is the Toeplitz autocorrelation matrix of the clutter. The use of ( ) parentheses indicates a continuous time signal and [ ] for a discrete time signal. In addition, we described all signals as a function of time, while its relationship to spatial distance is understood through the equation $\mathrm{c}=$ 


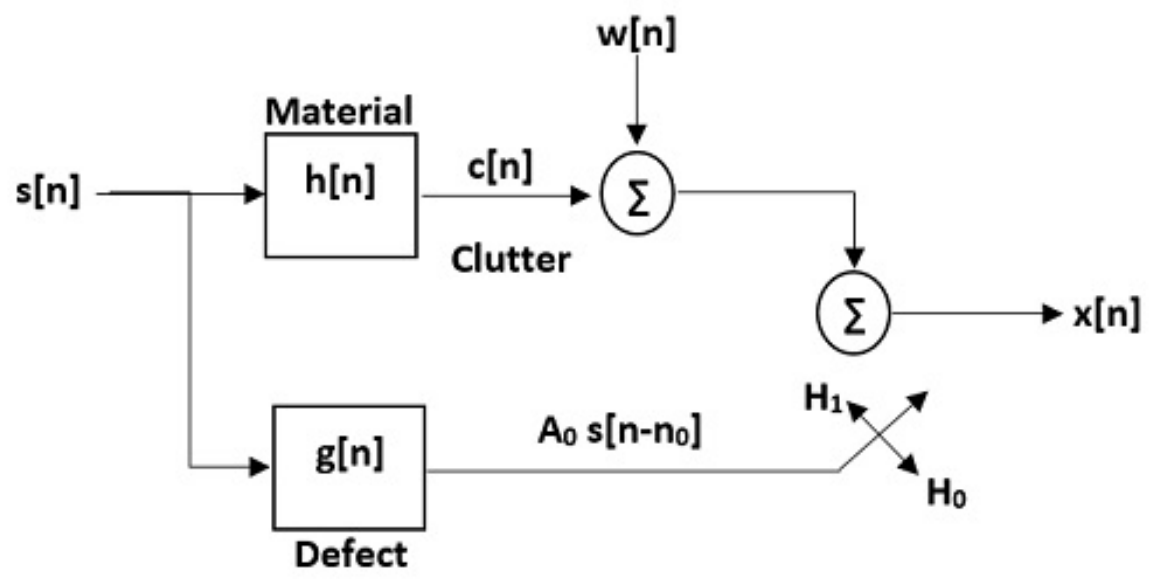

Figure 17: Modeling the received signal $x[n], s[n]$ is the ultrasonic transmitted pulse, $h[n]$ is a random LTI filter, $g[n]$ is the impulse response of the defect, $w[n]$ is the ambient white noise, $A_{0}$ and $n_{0}$ are the scale factor and delay that correspond to the target.

distance(meter) / time(second), where $\mathrm{c}$ is the speed of sound in the material.

\subsubsection{Model Review}

In [5] we showed how to construct a new physically motivated model for the grain scattering as a Gaussian wide sense stationary (WSS) random process, and we determined its mean, ACS, and its power spectral density (PSD). We showed that the process $h[n]$ can be thought of as the product of two independent processes $a[n]$, and $u\left[n-m_{0}\right]$ (normalized), that is $h[n]=a[n]\left(u\left[n-m_{0}\right]-\mu_{u}\right)$. The process $a[n]$ is assumed to be an IID Gaussian random process with a $\operatorname{PDF} \mathbf{a} \sim \mathcal{N}\left(\mathbf{0}, \sigma_{A}^{2} \mathbf{I}\right)$. We assumed that the process $u[n]$ is an impulse train process with impulses that are randomly distributed as described next. Referring to Fig. 18, without loss of generality, let $I_{i}=\{n: i M \leq n \leq i M+(M-1)\}$. Then:

1. In each interval $I_{i}$, a position is chosen at random for the spike. That is to say $\xi_{i} \in[0, M-1]$, where $\xi_{i}$ is a discrete random variable taking on integer values with $\xi_{i} \sim \mathcal{U}[0, M-1]$, and $\mathcal{U}$ denotes the discrete uniform distribution. 
2. All spike locations $\xi_{i}$ 's are $I I D$. This forms the process $u[n]$.

3. Then the process $u[n]$ is shifted to the right by $m_{0}$ samples, where $m_{0} \sim$ $\mathcal{U}[0, M-1]$.

4. The random shift $m_{0}$ is independent of all $\xi_{i}$ 's, which leads to our new process $v[n]=u\left[n-m_{0}\right]$.

In the above assumptions $M$ is a parameter that represents a number of samples, and is proportional to the average grain size of the material under testing, as well as the sampling rate and equal to $M=\left\lceil\frac{2 D}{c T_{s}}\right\rceil$, where $\lceil$ indicates the round up to the nearest integer and $D$ is the average grain size diameter [5]. We showed that our model incorporates the single scatterer restriction per average grain size and utilizes the average grain size explicitly. It also accounts for the random shape and orientation of the grains. The random shift $\xi_{i}$ accounts for the different orientation and shape of the grains; therefore the scatterer center will vary from grain to grain. As for the clutter $c[n]$, the input to the random filter $h[n]$ is assumed to be the transmitted pulse $s[n]$, so that $c[n]=s[n] * h[n]$, where $*$ denotes the convolution operator. Since the convolution is a commutative operation, we can write $c[n]=h[n] * s[n]$, where the filter input is now $h[n]$ and the filter impulse response is $s[n]$. Since we assumed $h[n]$ is a WSS Gaussian random process, let us denote its distribution by $\mathcal{N}\left(\mathbf{0}, \mathbf{C}_{h}\right)$, where $\mathbf{C}_{h}$ is the Toepltiz autocorrelation matrix. We know that the distribution of $c[n]$ will also be a WSS Gaussian random process with ACS equal to

$$
r_{c}[k]=s[-k] * s[k] * r_{h}[k]
$$

and PSD equal to

$$
P_{c}(f)=|S(f)|^{2} P_{h}(f)
$$



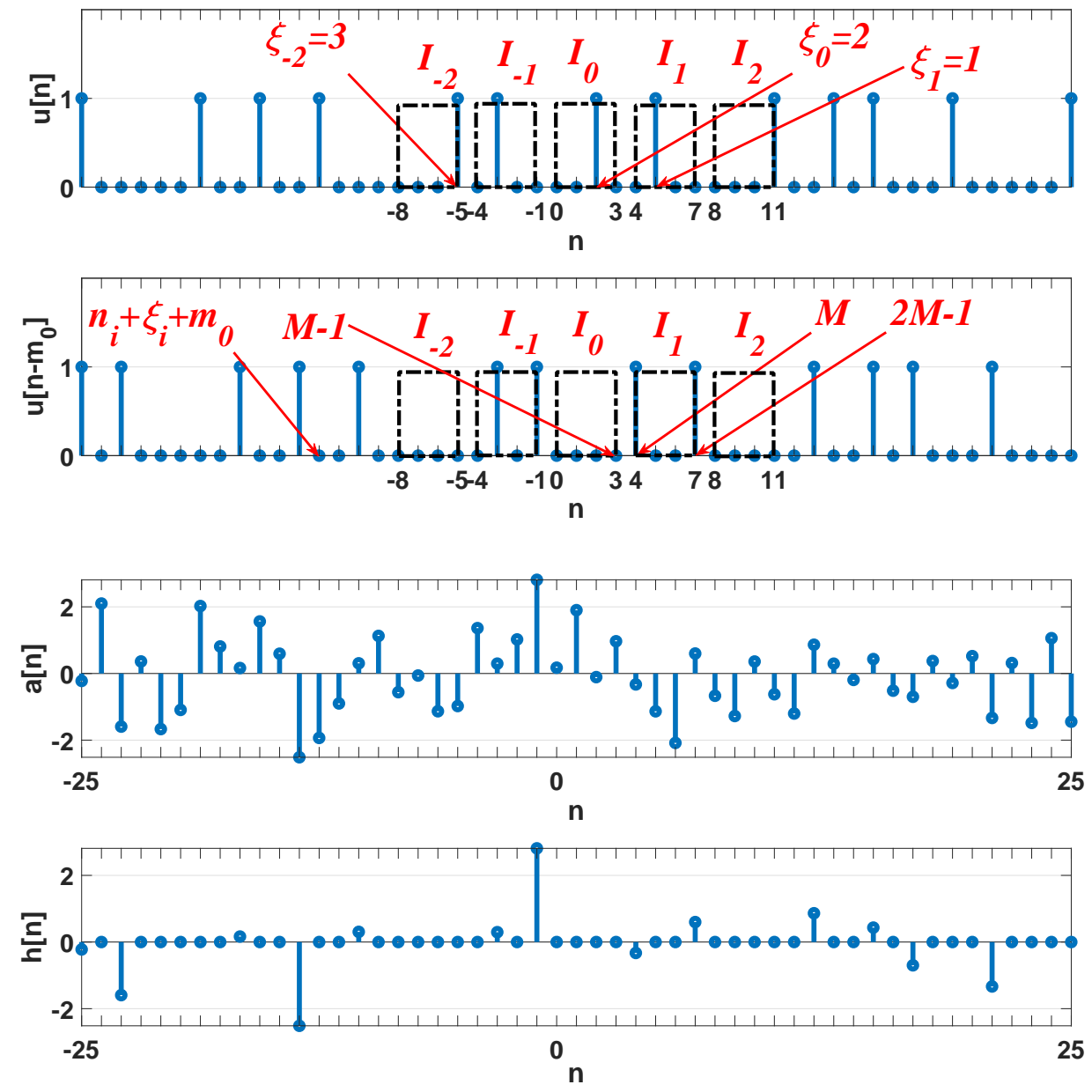

Figure 18: Realization portion of $u[n], u\left[n-m_{0}\right], a[n]$, and $h[n]=a[n]\left(u\left[n-m_{0}\right]-\right.$ $\left.\frac{1}{M}\right)\left(M=4\right.$ and $\left.m_{0}=2\right)$

where $-\frac{1}{2} \leq f \leq \frac{1}{2}, r_{h}[k]$ and $P_{h}(f)$ are the ACS and PSD for the process $h[n]$ respectively [11, p.602]. More details about the model assumptions and a full derivation of the mean, ACS, and PSD of $h[n]$ can be found in [5]. Letting $\alpha=$ $\frac{1}{M^{2}}(M-1) \sigma_{A}^{2}$, then the mean, ACS, and PSD of $h[n]$ is given by $\mu_{h}=0, r_{h}[k]=$ $\alpha \delta[k], P_{h}(f)=\alpha$ and for $c[n]$ using (12) and (13), we get

$$
r_{c}[k]=\alpha(s[-k] * s[k])
$$

and a PSD of

$$
P_{c}(f)=\alpha|S(f)|^{2} \quad-\frac{1}{2} \leq f \leq \frac{1}{2}
$$


Thus, we can denote the distribution of $\mathbf{c}$ as $\mathcal{N}\left(\mathbf{0}, \mathbf{C}_{c}\right)$, where the elements of $\mathbf{C}_{c}$ can be calculated from $\left[\mathbf{C}_{c}\right]_{i j}=\alpha[s[k] * s[-k]]_{k=i-j}$. As shown in (14), the clutter matrix $\mathbf{C}_{c}$ depends on the parameters $\sigma_{A}^{2}$ and $M$ (actually only on the $\alpha$ parameter), and the transmitted ultrasonic signal $s[n]$. As indicated, the process $h[n]$ has a flat spectrum so the output PSD $P_{c}(f)$ of the random LTI system is just a scaled version of the energy spectral density (ESD) of the transmitted pulse $s[n]$ (shown in (15)). Moreover, the Gaussian assumption for the clutter $c[n]$ is a reasonable one when the number of grains is large enough for the central limit theory to hold [12]. Since the clutter matrix depends on the transmitted signal $s[n]$, then finding the optimal transmitted signal to increase detectability is desired.

\subsection{Detector Performance}

Our model implementation in [5] leads to an easily implemented optimal detector, under the assumption of a known defect signal $A_{0} s\left[n-n_{0}\right]\left(A_{0}\right.$ and $n_{0}$ are known). To start, we assume that the signal model is known, the observation window is aligned with the actual defect location ( $n_{0}$ is known), and the amplitude is known and positive ( $A_{0}>0$ and known). In this manner, the problem becomes a classical known deterministic signal in colored Gaussian noise. For this problem, the optimal detector that maximizes the probability of detection for a fixed probability of false alarm, can be obtained by using the Neyman-Pearson criterion [4]. Referring to Fig. 17 and (11), the optimal detector decides a defect is present $\left(\mathcal{H}_{1}\right)$ if $L_{G}(\mathbf{x})=\frac{p\left(\mathbf{x} ; \mathcal{H}_{1}\right)}{p\left(\mathbf{x} ; \mathcal{H}_{0}\right)}>\gamma$, where $L_{G}(\mathbf{x})$ is the likelihood ratio function, $p\left(\mathbf{x} ; \mathcal{H}_{1}\right)$ and $p\left(\mathbf{x} ; \mathcal{H}_{0}\right)$ are the probability density functions under $\mathcal{H}_{1}$ and $\mathcal{H}_{0}$ respectively, and $\gamma$ is a threshold constant. We have

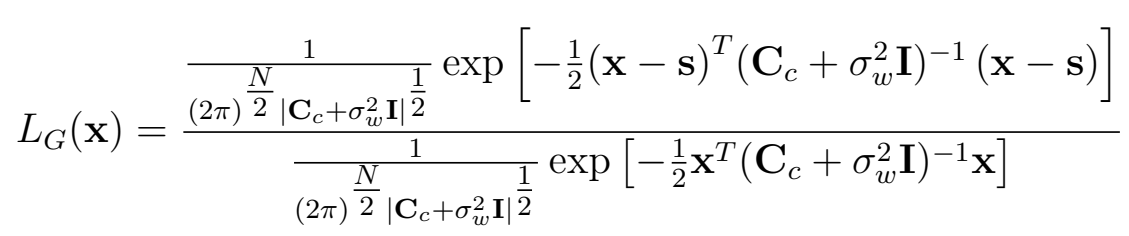


where $\mathbf{s}=\left[\begin{array}{llll}A_{0} s\left[n_{0}\right] & A_{0} s\left[n_{0}+1\right] & \cdots & A_{0} s\left[n_{0}+L-1\right]\end{array}\right]^{T}$, and $L$ is the number of samples that corresponds to the transmitted pulse length. Let $\mathbf{R}=\mathbf{C}_{c}+\sigma_{w}^{2} \mathbf{I}$. After simplifying the above expression we get

$$
T(\mathbf{x})=\mathbf{x}^{T} \mathbf{R}^{-1} \mathbf{s}_{0}
$$

where $T(\mathbf{x})$ is called the test statistic, and $\mathbf{s}_{0}=$ $\left[\begin{array}{llll}s\left[n_{0}\right] & s\left[n_{0}+1\right] & \cdots & s\left[n_{0}+L-1\right]\end{array}\right]^{T} \cdot \quad$ This form of the test statistic is called the generalized matched filter $(\mathrm{GMF})$ (we will call it $T_{G M F}(\mathbf{x})$ ), since we are correlating the received signal $\mathbf{x}$ with a whitening version $\mathbf{R}^{-1} \mathbf{s}$ of the transmitted pulse. The distribution of the test statistic for the GMF is the following

$$
T_{G M F}(\mathbf{x}) \sim \begin{cases}N\left(0, \mathbf{s}_{0}^{T} \mathbf{R}^{-1} \mathbf{s}_{0}\right) & \text { under } \mathcal{H}_{0} \\ N\left(A_{0} \mathbf{s}_{0}^{T} \mathbf{R}^{-1} \mathbf{s}_{0}, \mathbf{s}_{0}^{T} \mathbf{R}^{-1} \mathbf{s}_{0}\right) & \text { under } \mathcal{H}_{1}\end{cases}
$$

For this test statistics the performance of the GMF is

$$
\begin{gathered}
P_{F A}=Q\left(\frac{\gamma^{\prime}}{\sqrt{\mathbf{s}_{0}^{T} \mathbf{R}^{-1} \mathbf{s}_{0}}}\right) \\
P_{D}=Q\left(Q^{-1}\left(P_{F A}\right)-A_{0} \sqrt{\mathbf{s}_{0}^{T} \mathbf{R}^{-1} \mathbf{s}_{0}}\right)
\end{gathered}
$$

where the threshold can be found as $\gamma^{\prime}=Q^{-1}\left(P_{F A}\right) \sqrt{\mathbf{s}_{0}^{T} \mathbf{R}^{-1} \mathbf{s}_{0}}, P_{F A}$ is the probability of false alarm, $P_{D}$ is the probability of detection, and $Q()$ is the right-tail probability of standard normal Gaussian. From (16) the probability of detection, for a given probability of false alarm, depends on $d_{G M F}^{2}=A_{0}^{2} \mathbf{s}_{0}^{T} \mathbf{R}^{-1} \mathbf{s}_{0}$, where $d_{G M F}^{2}$ is called the deflection coefficient. Let $C N R(d B)=10 \log 10\left(\frac{r_{c}[0]}{r_{w}[0]}\right)$, where $r_{c}[0]$ is the total average power of the clutter noise and $r_{w}[0]$ is the total average power of the ambient white noise. We know that $r_{c}[0]=\alpha \mathcal{E}$ and $r_{w}[0]=\sigma_{w}^{2}$, where $\mathcal{E}$ is the energy of the transmitted ultrasonic pulse and $\alpha=\frac{1}{M^{2}}(M-1) \sigma_{A}^{2}$. The 
term $C N R(\mathrm{~dB})$ is what is commonly called clutter to ambient noise ratio. To make a fair comparison between the different transmitted signals, we impose the constraint for the signal energy to be equal for all of them.

\subsection{Maximization of The Deflection Coefficient}

From section 3 it was shown that the performance of the detector depends on the deflection coefficient $d_{G M F}^{2}=A_{0}^{2} \mathbf{s}_{0}^{T} \mathbf{R}^{-1} \mathbf{s}_{0}$. Hence, to find the optimal signal we need to maximize the deflection coefficient,

$$
d_{G M F}^{2}=\mathbf{s}^{T}\left(\mathbf{C}_{c}+\sigma_{w}^{2} \mathbf{I}\right)^{-1} \mathbf{s}
$$

where $\mathbf{s}=[s[0] s[1] \ldots s[N-1]]^{T}$ is the transmitted signal, $\mathbf{C}_{c}$ is the clutter covariance matrix, and $\sigma_{w}^{2}$ is the ambient white Gaussian noise variance. The clutter covariance matrix is given by

$$
\left[\mathbf{C}_{c}\right]_{i j}=\alpha \sum_{n=0}^{N-|i-j|} s[n] s[n+|i-j|]=r_{c}[i-j]
$$

for $i=1,2, \ldots, N, j=1,2, \ldots, N$ and is a symmetric Toeplitz autocorrelation matrix with $[i, j]$ element the scaled by $\alpha$ autocorrelation sequence of the signal, denoted by $r_{c}[i-j]$. The maximization is under the constraint that the signal energy is fixed, or $\mathcal{E}=\mathbf{s}^{T} \mathbf{s}$. Otherwise the maximum is obtained by letting the signal energy go to infinity. The proof is given in the appendix. The optimal signal for the above setup was found to be an impulse and equal to

$$
s[n]=\sqrt{\mathcal{E}} \delta[n]
$$

where $\delta[n]$ is the discrete-time delta function. An important conclusion, which results from the optimal transmitted signal being an impulse, is that $\mathbf{C}_{c}=r_{c}[0] \mathbf{I}=$ $\alpha \mathcal{E} \mathbf{I}$. Thus, the optimal signal is chosen and it is seen to result in the clutter being white. The clutter along with the ambient white noise is therefore white noise. Hence, the usual prewhitening of the generalized matched filter is no longer 
needed. In fact, the optimal detector when the optimal signal is used is just the standard matched filter. Interestingly, this type of result was originally stated by Van Trees [12], although without a proof but by applying it to a specific example. The maximum SNR is $d_{M A X}^{2}=\frac{\mathcal{E}}{\alpha \mathcal{E}+\sigma_{w}^{2}}$ (see the appendix).

\subsection{Optimal Signal Design}

To design the optimal signal we need to keep in mind that a signal with an impulsive ACS is not realizable in practice and an alternative must be pursued. In this work, we will examine signals that fall into four different categories:

1. Linear Frequency Modulated (LFM) signal.

2. Non-Linear Frequency Modulated (NLFM) signals.

3. Phase Coded Modulated (PCM) signals.

\section{Other signals.}

Since any signal that falls into any of these categories will not have an impulsive ACS but a semi impulsive ACS, that will raise the question, which properties of the signal do we focus on? This question cannot be answered without the context of the problem we are trying to solve, since for different applications certain properties will be more important to emphasize on than others. Some of these properties are: sidelobes suppression level, doppler tolerance, peak to sidelobe ratio (PSR), mainlobe width, ISL, merit factor (MF), and many others. Moreover, many limitations govern the type and the shape of the signal that we can transmit in an NDT applications such as: the resolution requirements, available energy level, and available bandwidth. The doppler effect in NDT applications does not come from the fact that the target is moving since the defect is stationary, however, it

comes from the frequency-dependent attenuation that is inherited in the material 
and the defect size. Even though the model did not incorporate the frequencydependency it still captured the essence of the process that generated the clutter in the material and worked well on real ultrasonic data, which indicates robustness [5]. A simple model as ours, in most cases, will be less susceptible to changes in comparison to a more complex model which is very susceptible to even small changes. To that end we will explore these four categories.

\subsubsection{LFM Signal}

Linear frequency modulated signal is the first and most used signal in many applications, especially in radar applications [13], and has been used in NDT applications [10]. The signal consists of a rectangular pulse of a constant amplitude (we will assume to be one) and a duration of $T$ second, where the frequency is swept, either in decreasing or increasing order, linearly throughout the duration of the signal. We can write the LFM signal in a complex notation, which is also called the analytic form, as follows:

$$
s_{a}(t)=\operatorname{rect}\left(\frac{t}{T}\right) \exp \left(j 2 \pi m t^{2}\right) \exp \left(j 2 \pi\left(F_{c}-\frac{B}{2}\right) t\right)
$$

where $0 \leq t \leq T, m=\frac{B}{2 T}, F_{c}$ is the center frequency, $B$ is the bandwidth in $\mathrm{Hz}$, and

$$
\operatorname{rect}\left(\frac{t}{T}\right)=\left\{\begin{array}{cc}
1 & 0 \leq t<T \\
0 & \text { otherwise }
\end{array}\right.
$$

the complex envelope of the signal is

$$
\tilde{s}(t)=\operatorname{rect}\left(\frac{t}{T}\right) \exp \left(j 2 \pi m t^{2}\right) \exp \left(-j 2 \pi \frac{B}{2} t\right)
$$

and to get the real transmitted signal $s(t)$ we take the real part of the complex envelop $\tilde{s}(t)$. It is clear that the instantaneous frequency is indeed a linear function of time. The LFM signal has an advantage of being doppler tolerant and easy to 
generate in comparison with other signals of the same kind. Also, the power of the LFM signal is distributed evenly throughout the frequency spectrum, which allows for high spectral efficiency [14]. However, it suffers from high ACS sidelobes level. The ACS sidelobes level for the LFM signal could be reduced by shaping the power spectrum through the use of any of the suppression windows (Hann, Hamming, etc.) [15]. One draw back of using a suppression window is that variable amplitude requires linear power amplifiers which are less efficient than saturated power amplifiers [14].

\subsubsection{NLFM Signal}

Since the LFM signal suffers from high ACS sidelobes level, another method for shaping the power spectrum is to deviate from the constant rate of frequency change and to spend more time at frequencies that need to be enhanced. This approach is called the non-linear frequency modulation [16]. There are two methods to synthesize an NLFM signal. First, is called the stationary phase principle (SPP). Second, is called the explicit functions cluster method. In this paper, we will use the SPP method. A brief summary of the SPP is as follows:

1. Choose a signal $\mathrm{v}(\mathrm{t})$ that has the desired PSD, then take the square of the spectrum $V^{2}(f)$.

2. Perform the integration of the amplitude square spectrum $V^{2}(f)$. Since the ACS is the Fourier transform of the PSD the problem is the same as designing a signal with good sidelobe property (suppressed),

$$
T(f)=\int_{-\frac{B}{2}}^{\frac{B}{2}} k_{1} V^{2}(f) d f+k_{2}
$$

where $k_{1}$ and $k_{2}$ are constants that ensure that $T\left(\frac{-B}{2}\right)=\frac{-T}{2}$ and $T\left(\frac{B}{2}\right)=\frac{T}{2}$. Moreover, $T(f)$ is the time delay. 
3. Take the inverse of the time delay to get the instantaneous frequency function,

$$
f(t)=T^{-1}(f)
$$

4. Perform the integration of the instantaneous frequency to get the phase,

$$
\phi(t)=\int_{-\frac{T}{2}}^{\frac{T}{2}} f(\lambda) d \lambda
$$

5. Find the real NLFM signal,

$$
s(t)=a(t) \cos \left(2 \pi f_{c} t+\phi(t)\right)
$$

In designing NLFM signal it is recommended to keep the amplitude $a(t)$ constant, in this work we will assume $a(t)=1$ for the whole signal duration. For more details see [16],[17],[18] and the references within.

In the following subsections we will discuss two of the NLFM signals that are widely used in radar and can be used in NDT applications as well.

\section{Price NLFM}

Price in [19] has reached empirically a good NLFM signal. He combined both LFM and NLFM according to

$$
f(t)=\frac{t}{T}\left(B_{L}+B_{C} \frac{1}{\sqrt{1-\left(4 t^{2} / T^{2}\right)}}\right)
$$

where $\frac{-T}{2} \leq t \leq \frac{T}{2}, B_{L}$ is the total frequency sweep for the LFM, and $B_{C}$ is the total frequency sweep for NLFM. Using the SPP method we get the phase $\phi(t)$ then we can construct our real transmitted signal using $s(t)=\cos \left(2 \pi f_{c} t+\phi(t)\right)$. 


\section{Cook NLFM}

Cook in [20] used the SSP to design signals whose PSDs have the shape of $\cos ^{n}(f)$ for $n=1,2,3,4$. In our work, we took the case for $n=4$. The amplitude spectrum of the signal is approximated as:

$$
V^{2}(f)=\left[\cos \left(\frac{\pi f}{B}\right)\right]^{n}
$$

where $\frac{-B}{2} \leq f \leq \frac{B}{2}$, and the time delay function is

$$
T(f)=T\left[\frac{f}{B}+\frac{1}{2 \pi} \sin \left(\frac{2 \pi f}{B}\right)+\frac{2}{3 \pi} \cos ^{3}\left(\frac{\pi f}{B}\right) \sin \left(\frac{\pi f}{B}\right)\right]
$$

following the steps of the SPP we found the instantaneous frequency function $f(t)$ by inverting the time delay function $T(f)$ numerically. We then performed the integration numerically to find the phase $\phi(t)$. Finally, we construct the actual transmitted signal $s(t)=\cos \left(2 \pi f_{c} t+\phi(t)\right)$.

\subsubsection{PCM Signals}

The doppler intolerance that PCM signals suffer from make PCM signals suitable only for low doppler or static target applications. The PCM signals still can provide a good sidelobes level suppression in comparison to the LFM signal. Similar results have been shown in regards to ultrasound imaging [15]. We start with a signal of duration $T$ second, then the signal is divided into $\mathrm{K}$ segments of identical duration $t_{m}=T / K$ second, and each segment is assigned with a different phase value. The bandwidth is decided by the width of each segment that is $B=\frac{1}{t_{m}} \mathrm{~Hz}$. The complex envelope of the PCM signal is given by

$$
\tilde{s}(t)=\sum_{m=1}^{K} \tilde{s}_{m} r e c t\left[\frac{t-(m-1) t_{b}}{t_{b}}\right]
$$

where $\tilde{s}_{m}=\exp \left(j \phi_{m}\right)$ and the set of $K$ phases $\phi_{1}, \phi_{2}, \ldots ., \phi_{K}$ is the phase code associated with $\tilde{s}(t)$. To get the real transmitted signal $s(t)$ we take the real part 
of the complex envelope $\tilde{s}(t)$. There are a large number of ways to generate $\mathrm{K}$ different phases. The criteria for selecting a specific code depends on the resolution properties of the resulting waveform (shape of the ACS), frequency spectrum, and the ease in which the system can be implemented. Phase coded signals can be categorized into two categories: binary phase coded signals such as Baker and P1 codes. The other category is polyphase coded signals such as Chu, Golomb, Frank and P4 codes. In spite of this inconvenience, these waveforms have shown significant promise due to the fact that polyphase coded signals exhibit low sidelobes level without having to resort to a weighting window unlike the LFM signal and are also directly compatible with digital generation and compression [14]. The number of bits $K$ with the length of each bit will get the total duration of the signal that is

$$
T=K t_{m}
$$

In our paper we will examine two polyphase coded signals.

\section{Chu Signal}

Chu in [21] designed a polyphase coded signal where the phases are defined as

$$
\phi_{m}=\left\{\begin{array}{cl}
\frac{2 \pi}{K} r \frac{(m-1)^{2}}{2} & \text { Keven } \\
\frac{2 \pi}{K} r \frac{(m-1) m}{2} & \text { Kodd }
\end{array}\right.
$$

where $r$ is any integer prime to $K$. Then we constructed the complex envelop and took the real part of it to get our actual real transmitted signal $s(t)$.

\section{Golomb Signal}

Golomb polyphase coded signals [22] are specific cyclically shifted and decimated versions of the Zadoff-Chu coded signals. Golomb code phases are defined 
as

$$
\phi_{m}=\frac{2 \pi}{K} r \frac{(m-1) m}{2}
$$

where $r$ is any integer relatively prime to $K$. Same as the Chu signal, we constructed the complex envelop and took the real part of it to get our actual real transmitted signal $s(t)$.

\subsubsection{Other Signals}

In this section we will discuss two other signals. The two signals come from the idea of minimizing a cost function, in this case the ISL function. Since the optimal signal is found to be an impulse and as a result it has an impulsive ACS, then a good design choice is a signal with good sidelobe suppression properties. If the $r_{s}[k]$ represents the ACS for the real transmitted signal $s[n]$ then the sidelobe is defined as all the ACS values at $k \neq 0$ and the mainlobe at $k=0$, hence, we need $\left\{r_{s}[k]\right\}_{k \neq 0}$ as small as possible. Here we will focus on the ISL metric, which is defined as

$$
I S L=\sum_{\substack{k=-(N-1) \\ k \neq 0}}^{(N-1)}\left|r_{s}[k]\right|^{2}
$$

Assume we have the complex sequence $\tilde{s}[n]$ (this will be the complex envelope of the real transmitted signal $s[n])$, the goal is to present an efficient computation algorithm to minimize the ISL metric or ISL-related metrics under the constraint of assuming the $|\tilde{s}[n]|=1$, which is an energy constraint that can be set to any energy level that is available. We start with,

\section{CAN Signal}

In this section we will present a cyclic algorithm for the local minimization of ISL-related metrics. The algorithm is called cyclic algorithm-new (CAN) and can be used for the local minimization of the ISL metric. CAN is based on the fast Fourier transform (FFT) operations and can be used for any values of $N$ up 
to $N \sim 10^{6}$ or even larger, where $N$ is the number of the signal samples. In [23] it was shown that

$$
\left|\sum_{n=1}^{N} \tilde{s}[n] e^{-i w n}\right|^{2}=\sum_{k=-(N-1)}^{(N-1)} r_{\tilde{s}}[k] e^{-i w k} \triangleq \Phi(w) .
$$

In [14] using (18), it was shown that the ISL defined in (17) can be represented in the frequency domain as follows

$$
I S L=\frac{1}{2 N} \sum_{p=1}^{2 N}\left[\Phi_{p}(w)-N\right]^{2}
$$

where $w_{p}$ are the Fourier frequencies and equal to $w_{p}=\frac{2 \pi}{2 N} p, p=1, \ldots \ldots, 2 N$. Using the periodogram representation for $\Phi(w)$ the problem of minimizing the ISL is similar to minimization of the following frequency domain function

$$
\sum_{p=1}^{2 N}\left[\left.|| \sum_{n=1}^{N} \tilde{s}[n] e^{-i w n}\right|^{2}-N\right]^{2}
$$

The similarity result has an intuitive interpretation: minimizing the ISL makes the sequence behave like a white noise, hence, its periodogram should be constant in the frequency domain [14]. To point out, the above function is a quartic function of $\tilde{s}[n]$. However, it can be shown that minimization (19) with respect to $\tilde{s}[n]$ is "almost equivalent" to a simpler problem whose quadratic with respect of $\tilde{s}[n]$

$$
\min _{\{\tilde{s}[n]\}_{n=1}^{N}:\left\{\psi_{p}\right\}_{p 1}^{2 N}} \sum_{p=1}^{2 N}\left|\sum_{n=1}^{N} \tilde{s}[n] e^{-i w_{p} n}-\sqrt{N} e^{i \psi_{p}}\right|^{2} .
$$

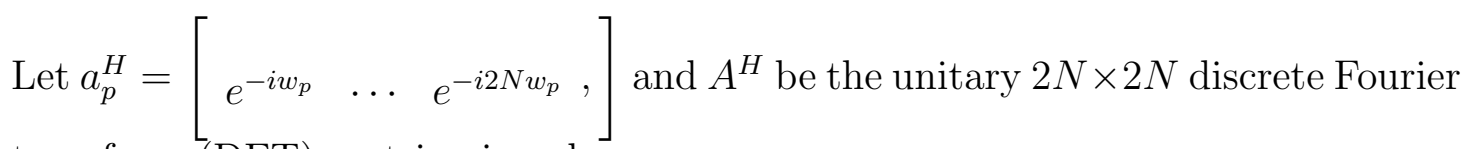
transform (DFT) matrix given by

$$
A^{H}=\frac{1}{\sqrt{2 N}}\left[\begin{array}{c}
a_{1}^{H} \\
\vdots \\
a_{2 N}^{H}
\end{array}\right]
$$


then the function in (20) can be written as $\left\|A^{H} \tilde{s}-\sqrt{N} v\right\|$, where $v=$ $\frac{1}{\sqrt{2}}\left[\begin{array}{lll}e^{i \psi_{1}} & \cdots & e^{i \psi_{2 N}}\end{array}\right]$. For a given sequence $\tilde{s}[n]$ the CAN algorithm minimizes the above function with respect to $\psi_{p}$, let $f=A^{H} z$ denotes the FFT of $z$; then $\psi_{p}=\arg \left(f_{p}\right)$ for $p=1, \ldots \ldots, 2 N$. Now letting $g=A v$ represent the inverse-FFT (IFFT) of $v$. Because $\left\|A^{H} z-v\right\|^{2}=\|z-A v\|^{2}$, it follows that the minimizing sequence $\tilde{s}[n]$ is given by

$$
\tilde{s}[n]=e^{i \arg \left(g_{n}\right)}
$$

for $n=1, \ldots \ldots, N$. A summary of the algorithm is given as follows

1. Step 1: Set $\{\tilde{s}[n]\}_{n=1}^{N}$ to random initial values (e.g., $\tilde{s}[n]$ can be set to $e^{j 2 \pi \phi(n)}$ where $\phi(n)$ are independent random variables uniformly distributed on $[0,2 \pi])$, or $\{\tilde{s}[n]\}_{n=1}^{N}$ can be initialized by a good existing sequence such as a Golomb sequence.

2. Step 2: Compute the $\left\{\psi_{p}\right\}_{p=1}^{2 N}$ that minimize the metric for $\{\tilde{s}[n]\}_{n=1}^{N}$ fixed at their most recent values.

3. Step 3: Compute the sequence $\{\tilde{s}[n]\}_{n=1}^{N}$ that minimizes the metric, under the constraint $|\tilde{s}[n]|=1$, or any energy level that is defined, for $\left\{\psi_{p}\right\}_{p=1}^{2 N}$ fixed at their most recent values.

4. Compute the sequence $\tilde{s}[n]_{n=1}^{N}$ that minimizes the metric, under the constraint $|\tilde{s}[n]|=1$, for $\left\{\psi_{p}\right\}_{p=1}^{2 N}$ fixed at their most recent values.

5. Step 4: Repeat steps 1-3 until a pre-specified stop criterion is satisfied, e.g., $\left\|\tilde{s}^{i}-\tilde{s}^{i+1}\right\|^{2}<\varepsilon$, where $\tilde{s}^{i}$ is the sequence obtained at the ith iteration and $\varepsilon$ is a predefined threshold, such as $10^{-3}$.

After we find the sequence $\tilde{s}[n]$ we can construct our real transmitted signal $s(t)$ in a similar fashion as the PCM signals. 
In [24] it was mentioned that the equivalence between the minimization of (19) and (20) actually are not exactly equivalent and they may have different solutions. So the point the CAN converges to is not necessary a local minimum or even stationary point of the original ISL metric minimization problem. However, in [24] it was shown numerically that CAN algorithm could generate sequences with good sidelobe suppression property. Also, CAN can be implemented using FFT, hence, can be used to design very long sequences.

\section{Monotonic Minimizer for Integrated Sidelobe Level Signal}

In [25] the authors designed a sequence with low sidelobes level via directly minimizing the ISL function in (17). Even though the design was considered for radar it is still applicable for NDT applications. The algorithm is called monotonic minimizer for integrated sidelobe level (MISL). The algorithm was derived based on the general framework of majorization-minimization (MM) algorithms. For a detail overview for the MM method readers are referred to [26], [27] and the references therein. In this section we will only summarize the algorithm in the following paragraph, readers who are interested in the details are referred to [25].

1. Require a sequence of length $\mathrm{N}$. Set $\mathrm{k}=0$, initialize $\tilde{s}^{0}$.

2. $P^{(k)}=\left|A^{H} \tilde{s}^{k}\right|^{2}$

3. $P_{\max }^{(k)}=\max _{P}\left\{P_{p}^{(k)}: p=1, \ldots, 2 N\right\}$.

4. $y=-A\left(\operatorname{Diag}\left(P^{k}\right)-P_{\max }^{(k)} I-N^{2} I\right) A^{H} \tilde{s}^{(k)}$

5. $\tilde{s}_{n}^{(k+1)}=e^{i \arg \left(y_{n}\right)}, n=1, \ldots, N$.

6. Repeat till convergence.

The advantage that MISL has over CAN is that MISL is guaranteed to converge to a stationary point and also MISL minimize the ISL metric directly, in comparison, 
the CAN algorithm minimizes an equivalent function that is not exactly equivalent. Similar to the CAN sequence to construct the real transmitted sequence $s(t)$ we used the PCM approach.

\subsection{Comparison Analysis}

The most commonly used excitation signal in practice is the GAMS signal. The reason for using the GAMS signal is that by using such a signal, for excitation, we utilize the available bandwidth from the probe in NDT applications more efficiently, since most commonly used probes in NDT applications are the ones with a Gaussian shape spectrum (impulse response). We will assume the phase is zero in this work.

\subsection{1 $d_{G M F}^{2}$ verses CNR and ROC curve}

In this section, we will make the comparison between the suggested signals themselves as well as the GAMS signal. According to our derivation in section 3, the detection performance depends on $d_{G M F}^{2}=A_{0}^{2} \mathbf{s}_{0}^{T} \mathbf{R}^{-1} \mathbf{s}_{0}$, hence, a plot between the CNR $(\mathrm{dB})$ verses the deflection coefficient in the region where we expect the noise to be will give a clear idea which signal has the best detectability performance. Moreover, we will use the ROC curve as another tool to test performance. In practice it is known that for NDT applications the CNR (dB) is between 25-35 $\mathrm{dB}$, hence, the region of interest. For NDT applications the bandwidth of the probe ranges from $100 \mathrm{KHz}$ to about $10 \mathrm{MHz}$ and a center frequency that falls in the same range. In this section, to make the comparison fair, all signals must have the same amount of energy $\mathcal{E}$, bandwidth $B$, signal duration $T$, and center frequency $F_{c}$. To that end, we chose a signal energy $\mathcal{E}=1$, bandwidth $B=4 \mathrm{MHz}$, signal duration $T=1 \mu \mathrm{sec}$, center frequency $F_{c}=5 \mathrm{MHz}$, and a sampling frequency rate of $F_{s}=100 \mathrm{MHz}$. The results are shown in Fig. 19 and a zoomed in look in 


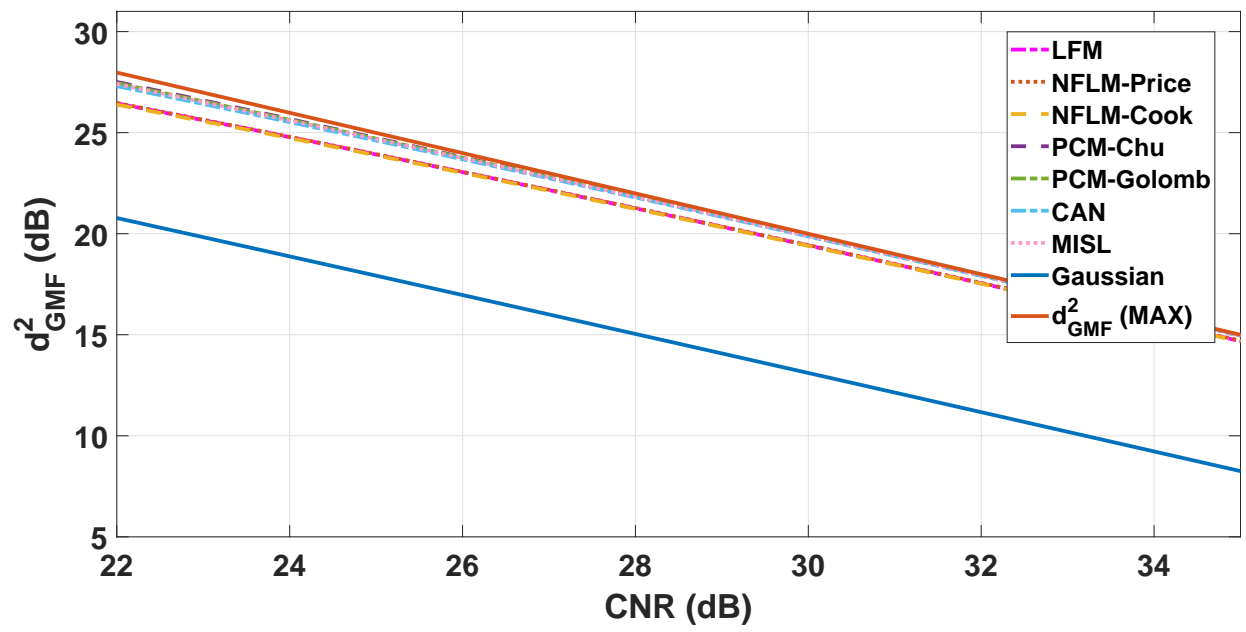

Figure 19: The deflection coefficient $d_{G M F}^{2}$ in $\mathrm{dB}$ vs clutter to ambient noise (CNR) in $\mathrm{dB}$

Fig. 20. It is clear from Fig. 19 that all suggested signals surpass the GAMS signal by almost $10 \mathrm{~dB}$. Also, as shown in both Fig. 19 and Fig. 20 the PCM signals (Chu, Golomb) and the signals generated from the CAN algorithm and the MISL algorithm show a little bit of an advantage over the LFM and NLFM signals, however, the advantage is not significant (it is less than $1 \mathrm{~dB}$ ). Keeping in mind, the LFM signal has the advantage of being more doppler tolerant in comparison to PCM and NLFM signals. Also, as shown in Fig. 19 and Fig. 20 all the proposed signals are less than $1 \mathrm{~dB}$ from the maximum value that the deflection coefficient can reach, which indicates that by using any of these signals we expect almost an optimal performance. The use of digital computers made the generation of any custom signals to be transferred through the probe in NDT applications possible, hence, the use of PCM signals, CAN signal, and MISL signal is possible.

For the ROC curve since the advantage of all the proposed signals over the LFM signal is less than $1 \mathrm{~dB}$ and since the LFM signal has the advantage of being doppler tolerant, it was sufficient to make the comparison only between the GAMS signal and the LFM signal in terms of the ROC curve for different signal energy 


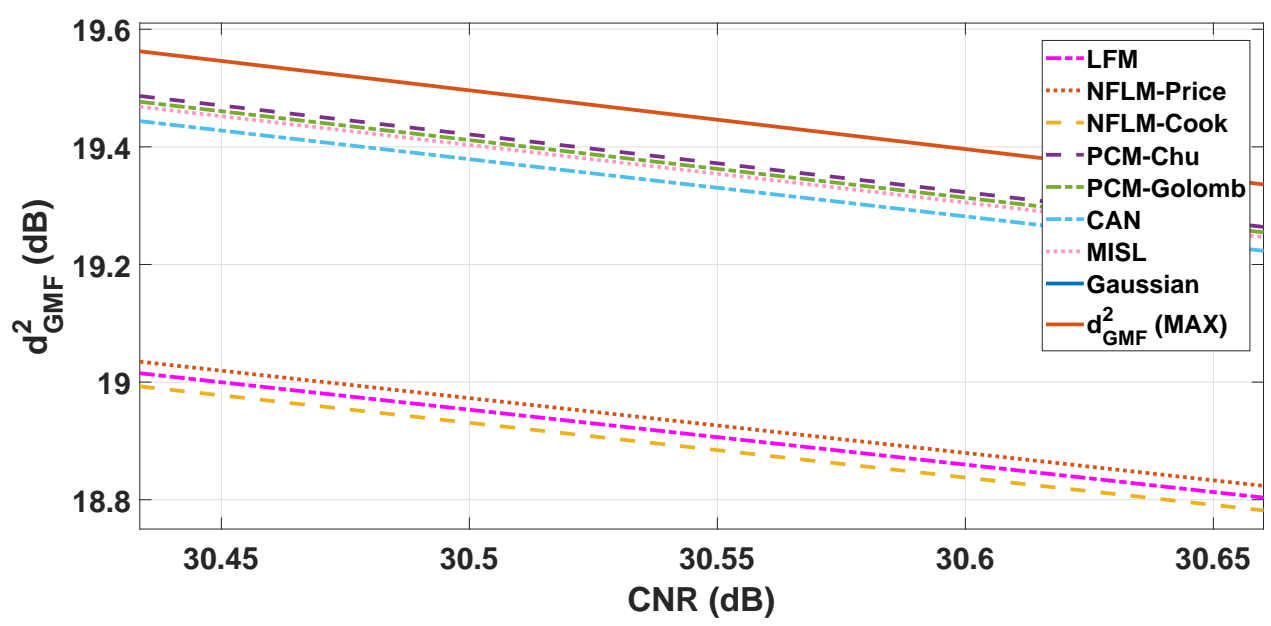

Figure 20: The deflection coefficient $d_{G M F}^{2}$ in $\mathrm{dB}$ vs clutter to ambient noise (CNR) in $\mathrm{dB}$

levels, which is indicated by the amplitude level. The results are shown in Fig. 21. It is clear from Fig. 21, since the amplitude levels reflect cases from low SNR to high SNR, that the LFM signal surpasses the GAMS signal in the low SNR region and improves as the SNR increases.

\subsubsection{Practical Detector-GLRT and Simulation}

Since we need to estimate $-\infty<A_{0}<\infty$ and $n_{0}$ in practice, a detector for the problem at hand was derived in [5]. We used the generalized likelihood ratio test (GLRT) approach, as a result we decide $\mathcal{H}_{1}$ (a defect is present) if

$$
T(\mathbf{x})=\max _{n_{0} \in[0: N-L]} \frac{\left(\mathbf{x}^{T} \mathbf{R}^{-1} \mathbf{s}_{0}\right)^{2}}{\mathbf{s}_{0}^{T} \mathbf{R}^{-1} \mathbf{s}_{0}}>\gamma^{\prime}
$$

where $\gamma^{\prime}$ is a threshold that depends on the optimality criterion of interest. To get the theoretical detection performance of the above test statistic is a difficult task, since we need to determine the PDF of the maximum of $N-L+1$ correlated Gaussian random variables and will not be pursued here. The option to determine the performance by simulation is a practical one and will be followed here. In this work, we determined the threshold by simulation, since the real test statistic PDF 

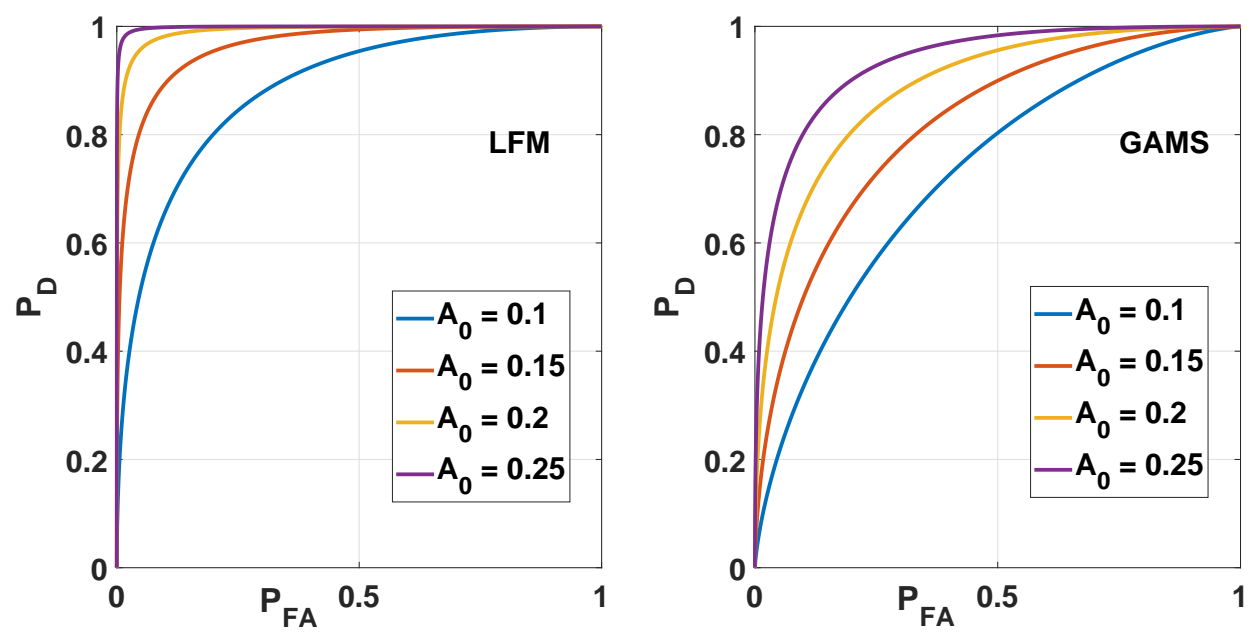

Figure 21: ROC curves for both LFM signal (Left) and the GAMS signal (Right) for different energy levels (Amplitude)

under $\mathcal{H}_{0}$ could not be achieved analytically. In practice, samples of defect free blocks can be first used to get an estimate for the PDF under $\mathcal{H}_{0}[5]$.

One of the main challenges that face researchers in NDT studies is the limited accessability to NDT equipment and specimen for testing. In addition, the lack of appropriate test specimens has made the development and evaluation of detection techniques even more challenging [28]. Another challenge in NDT research is the lack of a unified database that can be used to compare performances and the use of different metrics to evaluate these performances [5]. To that end, many models were provided for simulation purposes to resemble the clutter noise in materials and the defect reflected signal. By using these models many of the proposed algorithms can be tested. Such models can be found in [29].[28],[30],[31],[32]. Our proposed model in [5] even though was simple, it still worked well and showed excellent results on real ultrasonic data. The model was able to capture the essence of the clutter generating process in the material and showed robustness even if some of its assumptions were violated. Hence, the use of our model to simulate real data was pursued. Even though we are using simulated noise generated by our model for 
comparison between the LFM signal and the GAMS signal, the literature is filled with real-experimentations that prove that LFM, NLFM, and PCM signals have been used to enhance the SNR, resolution requirements, and detectibly through the efficient use of the available bandwidth in NDT applications, check [3],[6]-[10] and the references within. This work provides the statistical framework, a new model for the clutter noise, and a theocratical proof for the optimal signal design that is lacking in the literature. In addition, more signals other than the LFM, NLFM, and PCM are explored. To compare between the GAMS signal and the LFM signal we generated clutter noise using our model for both signals using the following parameters: a material with an average grain size of $150 \mu \mathrm{m}$ which corresponds to $M=5$, a speed of sound in the material equals 6000 meter/second similar to many stainless steel material, an ambient white noise with $\sigma_{w}^{2}=1 \times 10^{-5}$, and the amplitude variance of $\sigma_{A}^{2}=0.05$. These values reflect a $\mathrm{CNR}$ in the practical region of 25-35 dB. We chose a signal energy $\mathcal{E}=1$, bandwidth $B=2.5 \mathrm{MHz}$, transmitted signal duration $T=1 \mu \mathrm{sec}$, center frequency $F_{c}=5 \mathrm{MHz}$, a data record of 2000 samples (which correspond to $60 \mathrm{~mm}$ long block), and a sampling frequency rate of $F_{s}=100 \mathrm{MHz}$. We generated, as an example, four different realizations using both signals as shown in Fig. 22 and Fig. 23.

A defect was added to the noise realization at the labeled location as indicated in Fig. 22 and Fig. 23 (at $30 \mathrm{~mm}$ away from the surface of the block, which translate to $\left.n_{0}=1000\right)$. The energy of the reflected defect signals was set to 35 $\%$ of the transmitted signal energy. As for the threshold, since it depends on the optimality criterion of interest, we simulated 1000 of defect free realizations and picked the threshold that gives us a $P_{F A}=.01$, which is indicated with the red dotted line in both Fig. 24 and Fig. 25, and where $T_{n_{0}}(\mathbf{x})=\left.T(\mathbf{x})\right|_{n=n_{0}}=$ $\frac{\left(\mathbf{x}^{T} \mathbf{R}^{-1} \mathbf{s}_{0}\right)^{2}}{\mathbf{s}_{0}^{T} \mathbf{R}^{-1} \mathbf{s}_{0}}$. It is shown that for the LFM case we were able to detect the defect in all 

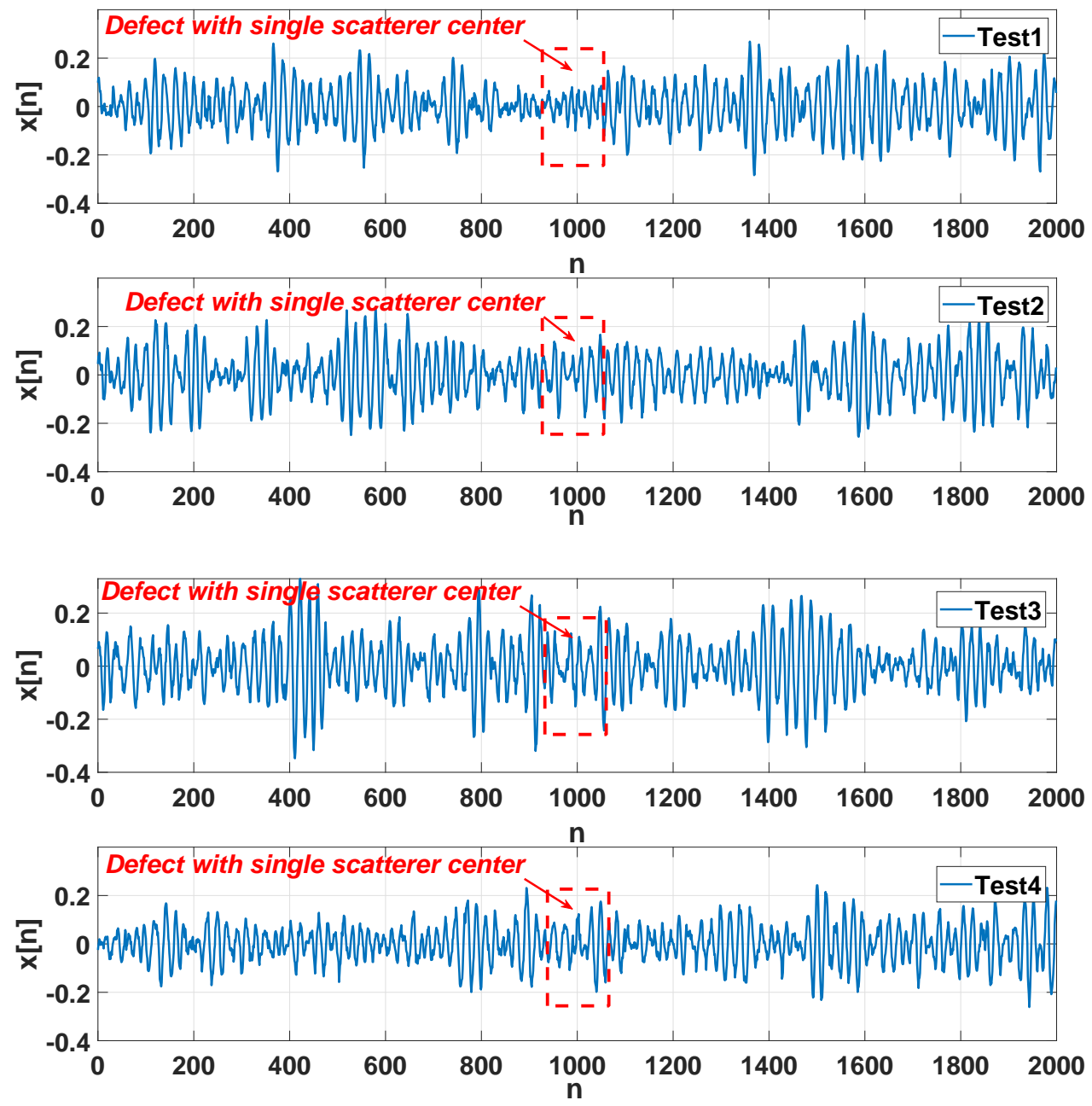

Figure 22: Received A-scan signals $x[n]$ using the LFM signal

samples, however, for the GAMS case we were able to only detect one defect for the specified $P_{F A}$. We continued our simulation by generating 1000 realizations with a defect at the same location as indicated previously. By choosing the mentioned threshold we got the following results

Table 2: Defect signal energy levels verses probability of detection $P_{D}$ for both LFM and GAMS signals

\begin{tabular}{|l|l|l|}
\hline Defect Signal Energy Level & $P_{D}$ LFM & $P_{D}$ GAMS \\
\hline $35 \%$ of $\mathcal{E}_{s}$ & 0.7090 & 0.1820 \\
\hline $50 \%$ of $\mathcal{E}_{s}$ & 0.9360 & 0.3120 \\
\hline $65 \%$ of $\mathcal{E}_{s}$ & 0.9860 & 0.4540 \\
\hline
\end{tabular}



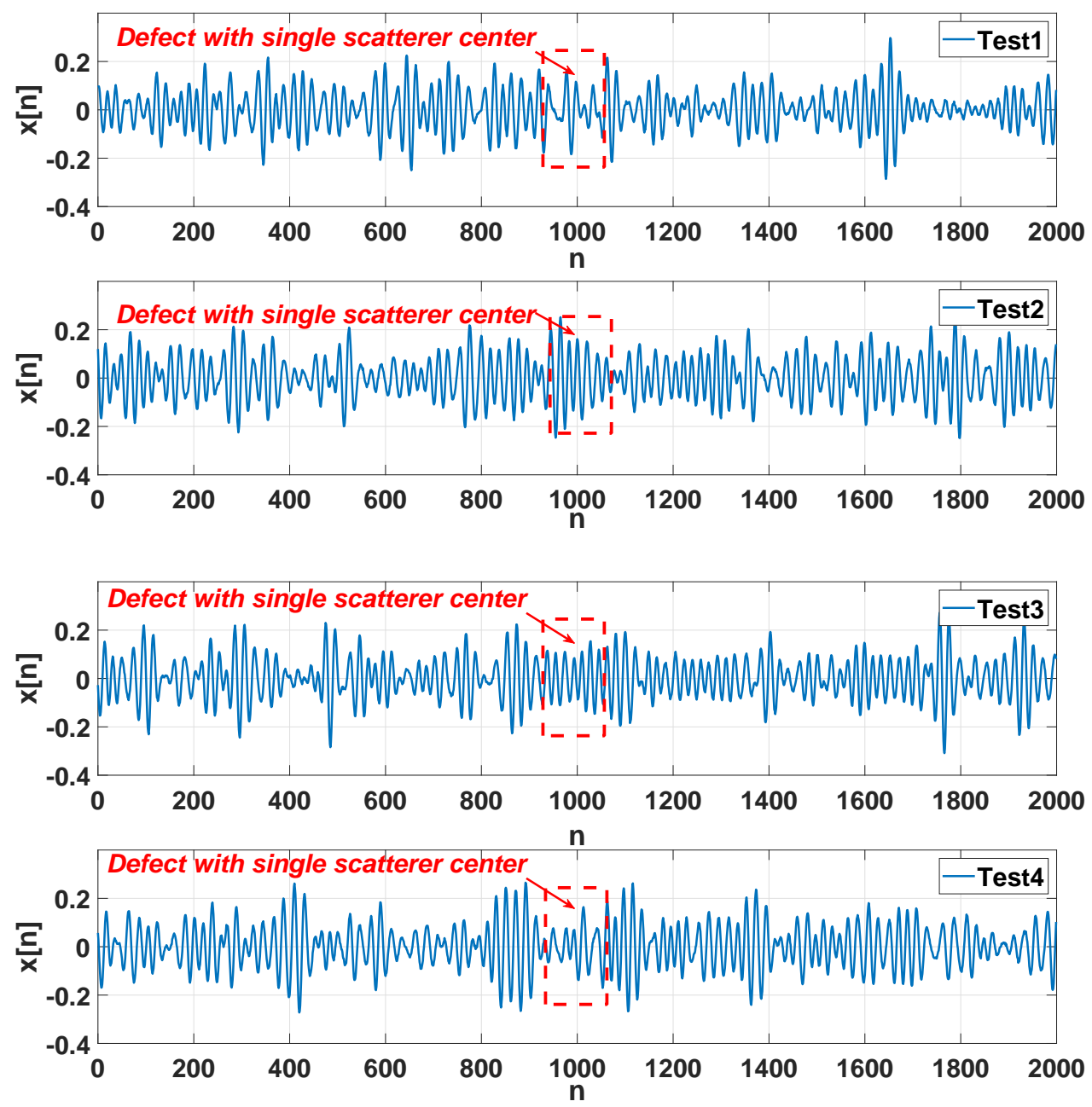

Figure 23: Received A-scan signals $x[n]$ using the GAMS signal

from the Table 2 it is clear that by using the LFM signal we were able to improve the detectability performance by an excellent margin, which is evident from the probability of detection each signal attained at the specified defect signal energy level.

\subsubsection{Comparison Using Multiscattering for A More Complicated Tar- get}

Another way to compare the LFM and GAMS signals is to test their ability to detect a defect with a more complicated geometrical shape. Since in many situations, a defect with a complicated geometrical shape, will cause the defect to 

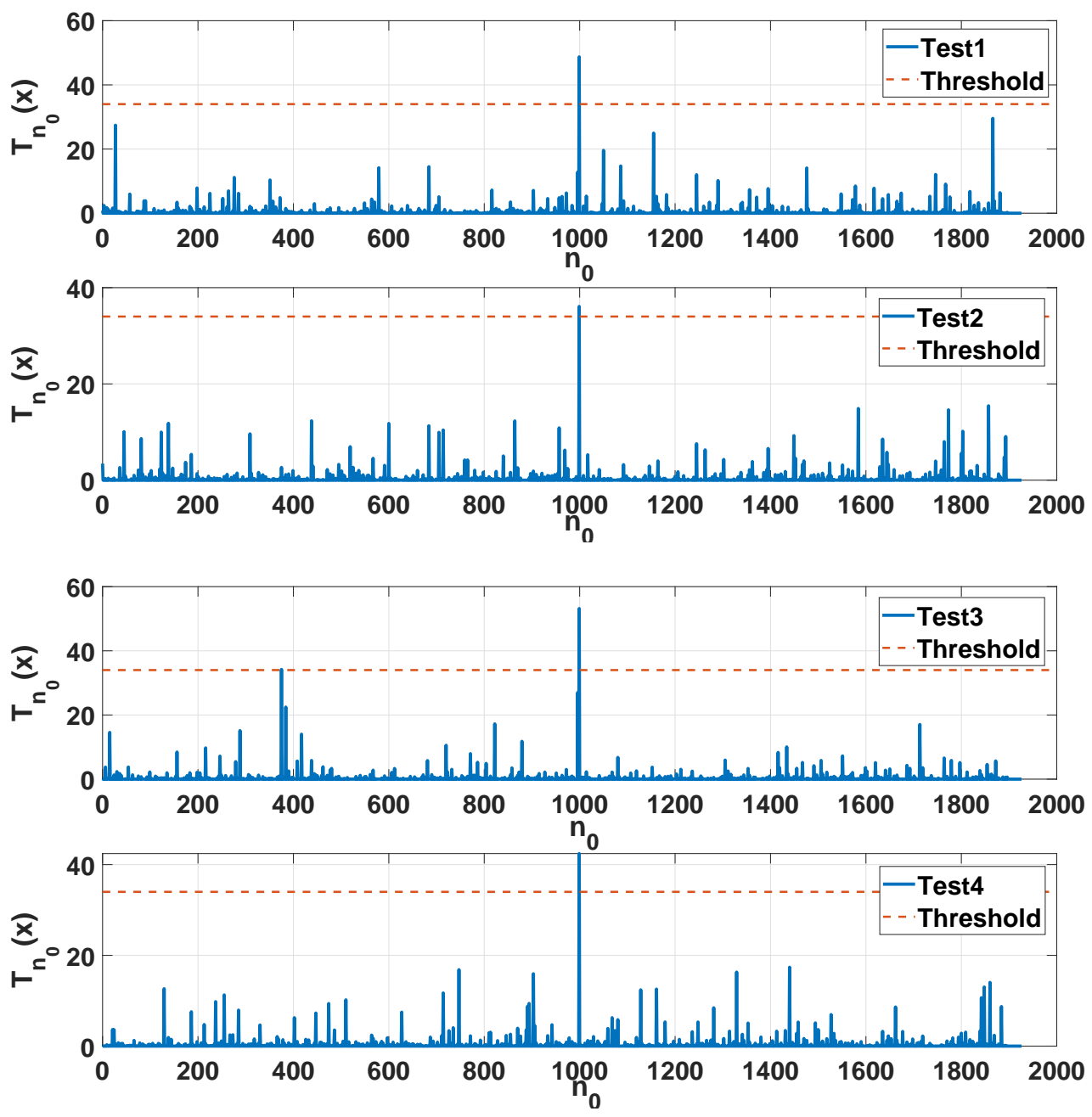

Figure 24: Test statistic $T_{n_{0}}(\mathbf{x})$ vs $n_{0}$ for GMF using LFM signal (GLRT Implementation)

have more than one scatterer surface in one resolution cell [5]. A resolution cell refers to the volume of the scanned material, illuminated by the transducer, within which only one defect scatterer can be identified [33]. In our work, the resolution cell is assumed to be approximately equal to the transmitted ultrasonic pulse $s[n]$ duration, which corresponds to $\mathrm{L}=100$ samples. Since the proposed speed of sound of the tested material is assumed $6000 \mathrm{~m} / \mathrm{sec}$; then the axial distance of one resolution cell is approximately $3 \mathrm{~mm}$. If the defect axial distance is contained in one resolution cell, this will cause the defect to have two or more scattering surfaces 

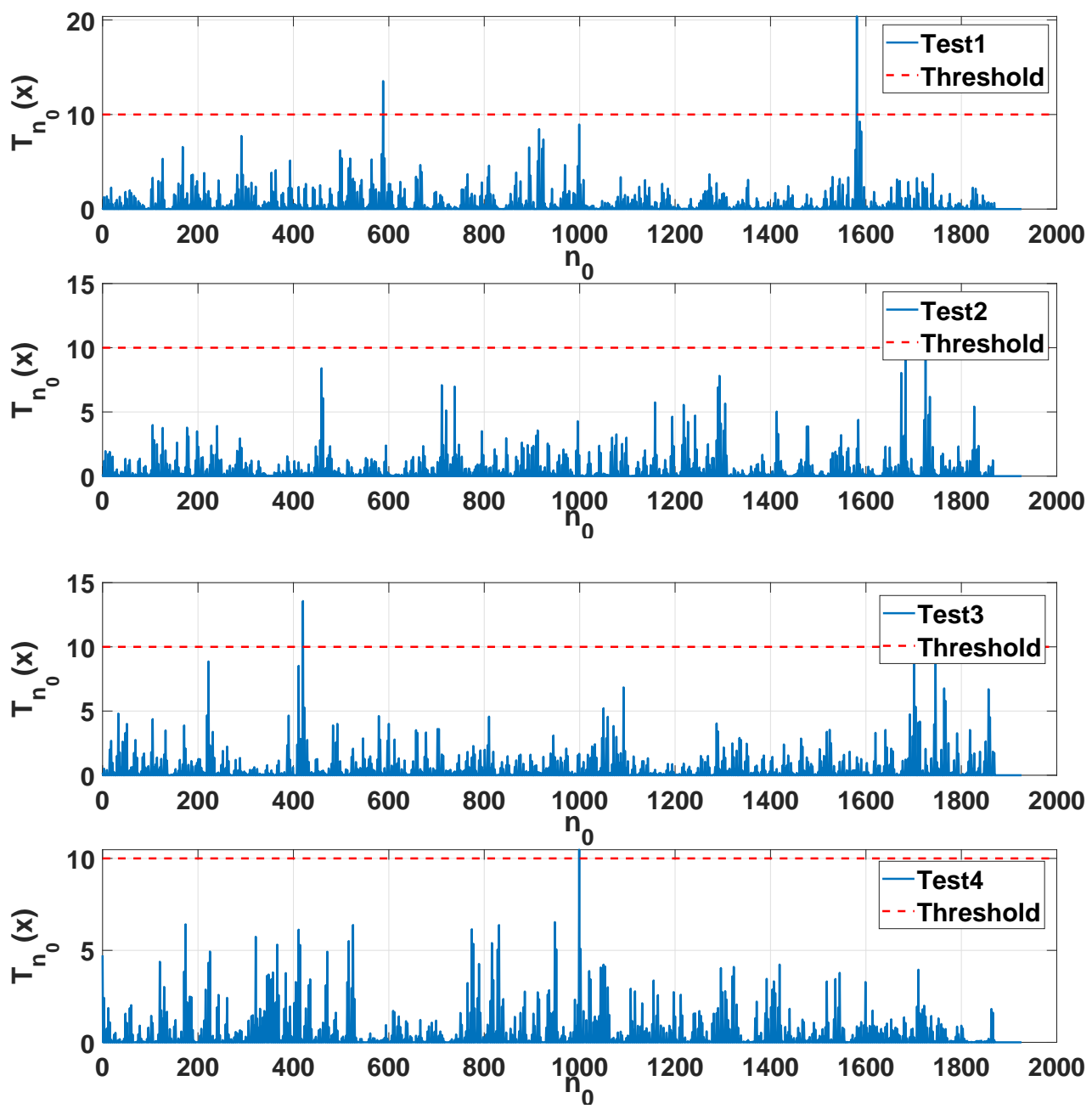

Figure 25: Test statistic $T_{n_{0}}(\mathbf{x})$ vs $n_{0}$ for GMF using GAMS signal(GLRT Implementation)

within one resolution cell. To simulate such a scenario, and since we assumed that the received signals are a distorted and delayed version of the transmitted signal, we generated two different reflected signals at two different energy level that are less than $3 \mathrm{~mm}$ apart. The energy level of the first defect reflected signal is assumed to be $50 \%$ of the transmitted signal energy $\mathcal{E}$ and the location is at $30 \mathrm{~mm}$ away from the surface of the block, which means $n_{0}=1000$ as before. The second reflected surface was simulated at different energy levels $25 \%, 35 \%, 50 \%$, and $65 \%$ of the transmitted signal energy $\mathcal{E}$. However, the location was varied to create the 

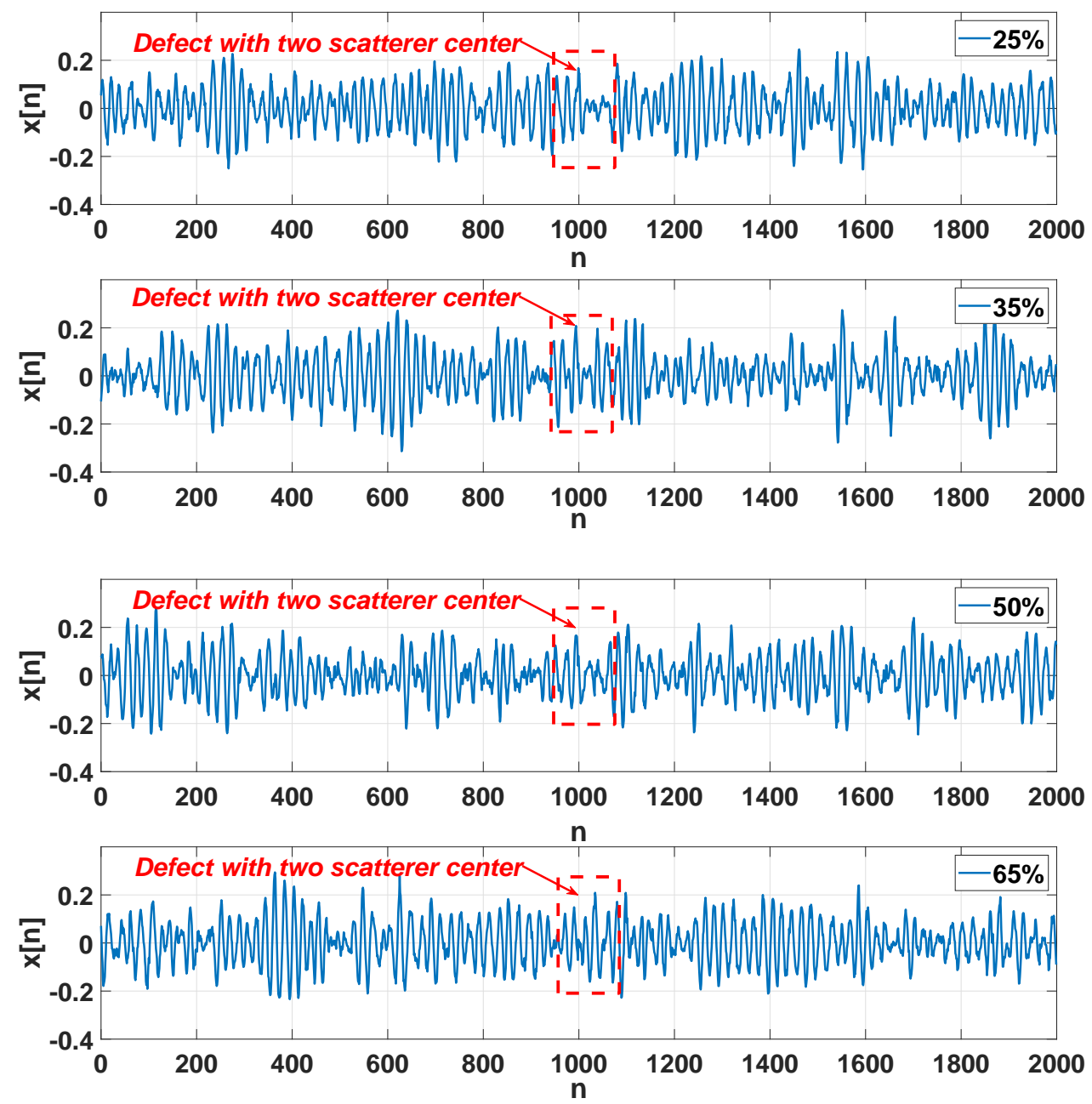

Figure 26: Received A-scan signals $x[n]$ using the LFM signal

difficult case where the two scatterer surface reflected signals combination is of the destructive type and not the constructive type to cause the overall defect reflected signal to be attenuated (but within $3 \mathrm{~mm}$ distance). The results after adding the hypothetical simulated reflected signals from the scatter surfaces are shown in Fig. 26 using the LFM signal and Fig. 27 using the GAMS signal. and the results for implementing the detector are shown in Fig. 12 and Fig. 13 As shown in Fig. 28 we were able to detect the defect in all four realizations by using the LFM signal, in comparison, we were only able to detect two out of four in the GAMS signal case. To quantify these results we simulated 1000 realizations for each case and by 

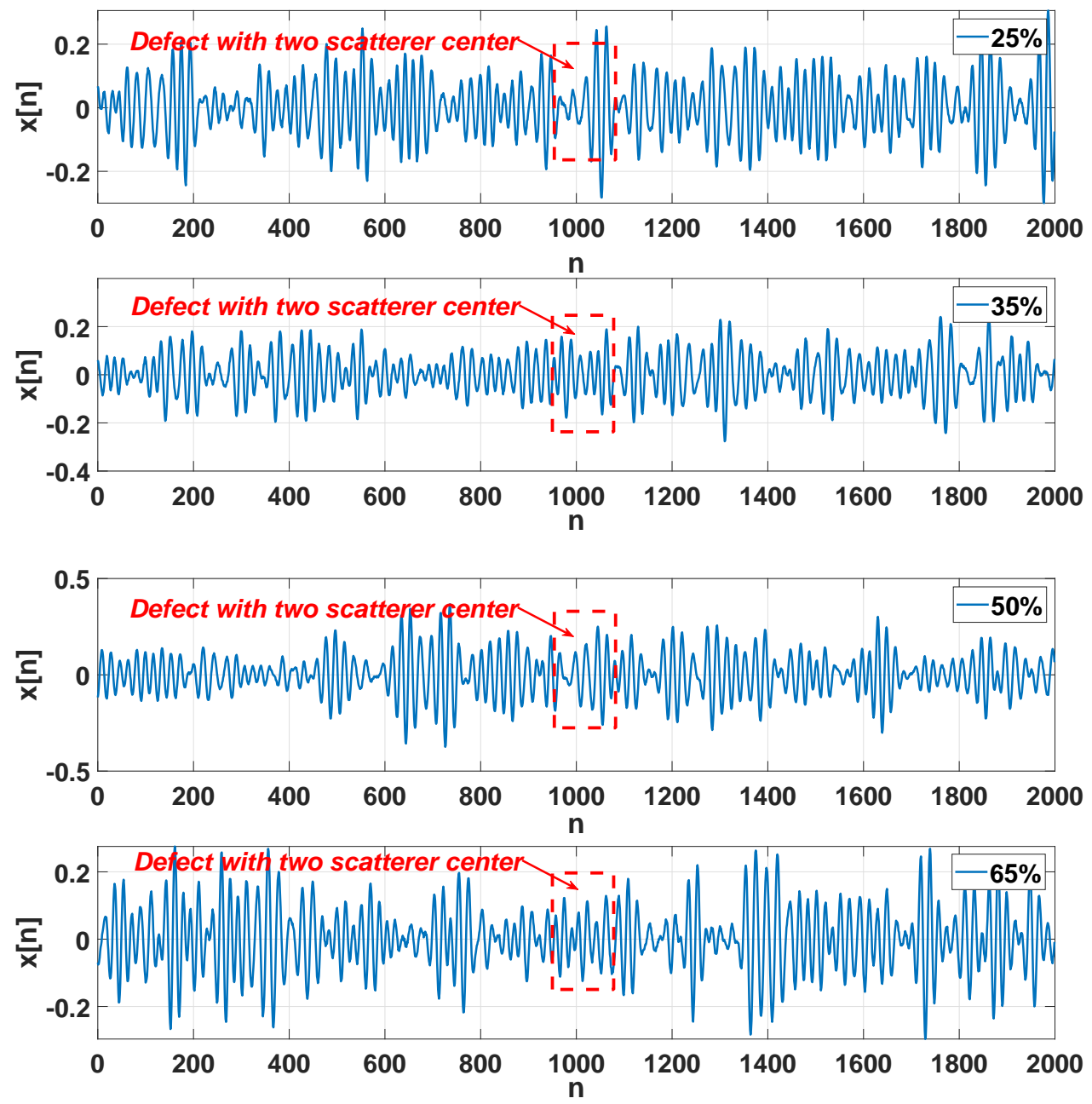

Figure 27: Received A-scan signals $x[n]$ using the GAMS signal

using the same $P_{F A}$ threshold we got the following results shown in Table 3 .

Table 3: Defect signal energy levels verses probability of detection $P_{D}$ for both LFM and GAMS signals for A More Complicated Target

\begin{tabular}{|l|l|l|}
\hline Defect Second Surface Signal Energy Level & $P_{D}$ LFM & $P_{D}$ GAMS \\
\hline $25 \%$ of $\mathcal{E}_{s}$ & 0.91 & 0.30 \\
\hline $35 \%$ of $\mathcal{E}_{s}$ & 0.89 & 0.26 \\
\hline $50 \%$ of $\mathcal{E}_{s}$ & 0.88 & 0.22 \\
\hline $65 \%$ of $\mathcal{E}_{s}$ & 0.84 & 0.16 \\
\hline
\end{tabular}

From Table 3 it is clear even with the deviation from the single scattering assumption and the two reflected signals being of the destructive type, the detector 

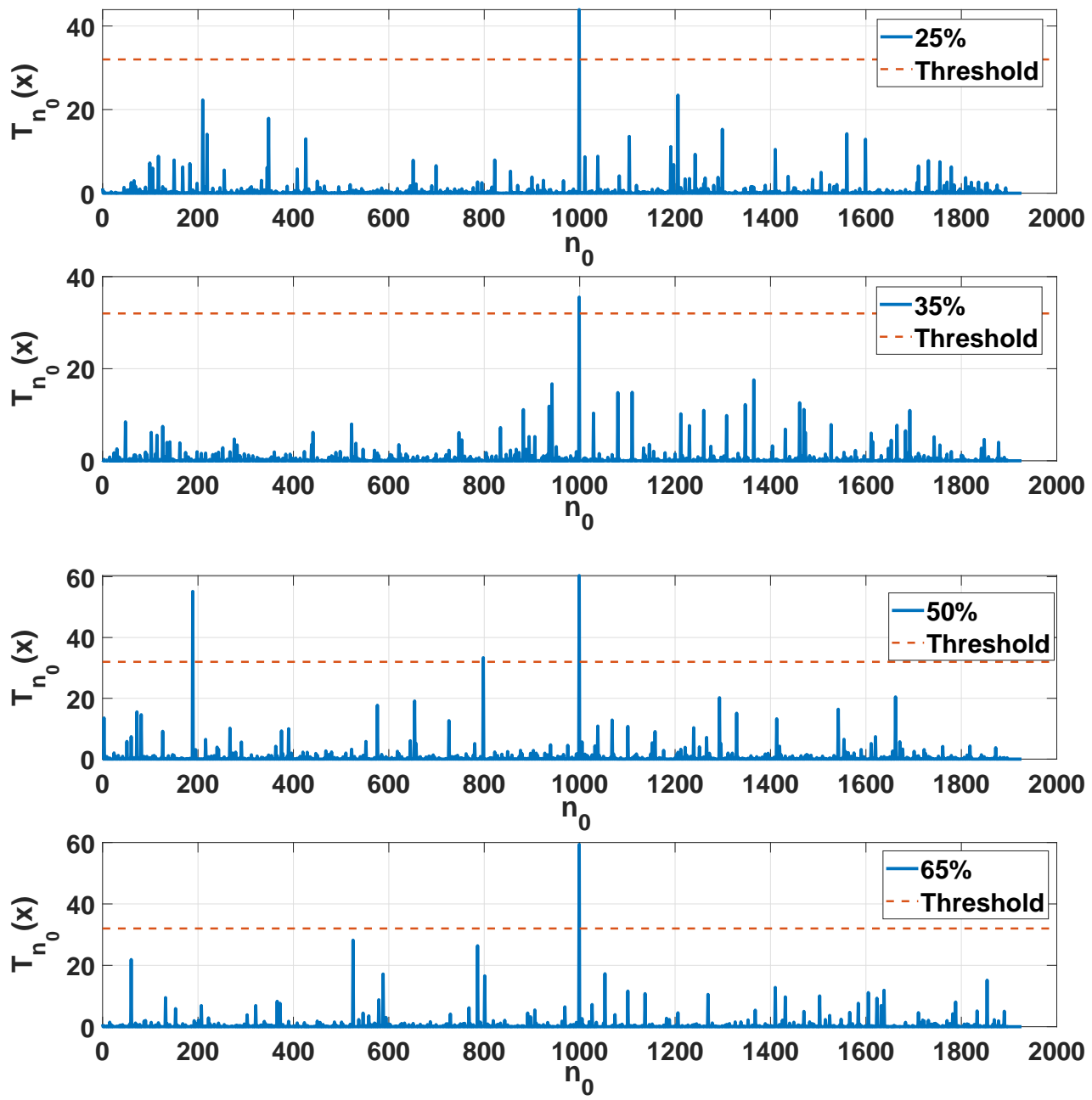

Figure 28: Test statistic $T_{n_{0}}(\mathbf{x})$ vs $n_{0}$ using LFM signal (GLRT Implementation)

performance for the LFM signal outperformed the GAMS signal. In addition, the introduce of the second reflected signal degraded the performance of the detector for both signals but it still worked well for the LFM with a $P_{D}=0.84$ in comparison of $P_{D}=0.16$ for the GAMS signal.

\subsection{Conclusion}

A continuation of the work on our new physically motivated model for the clutter noise has been done. A new proof, is lacking in the literature, answering the question of the optimal signal to be used in NDT session has been presented. 

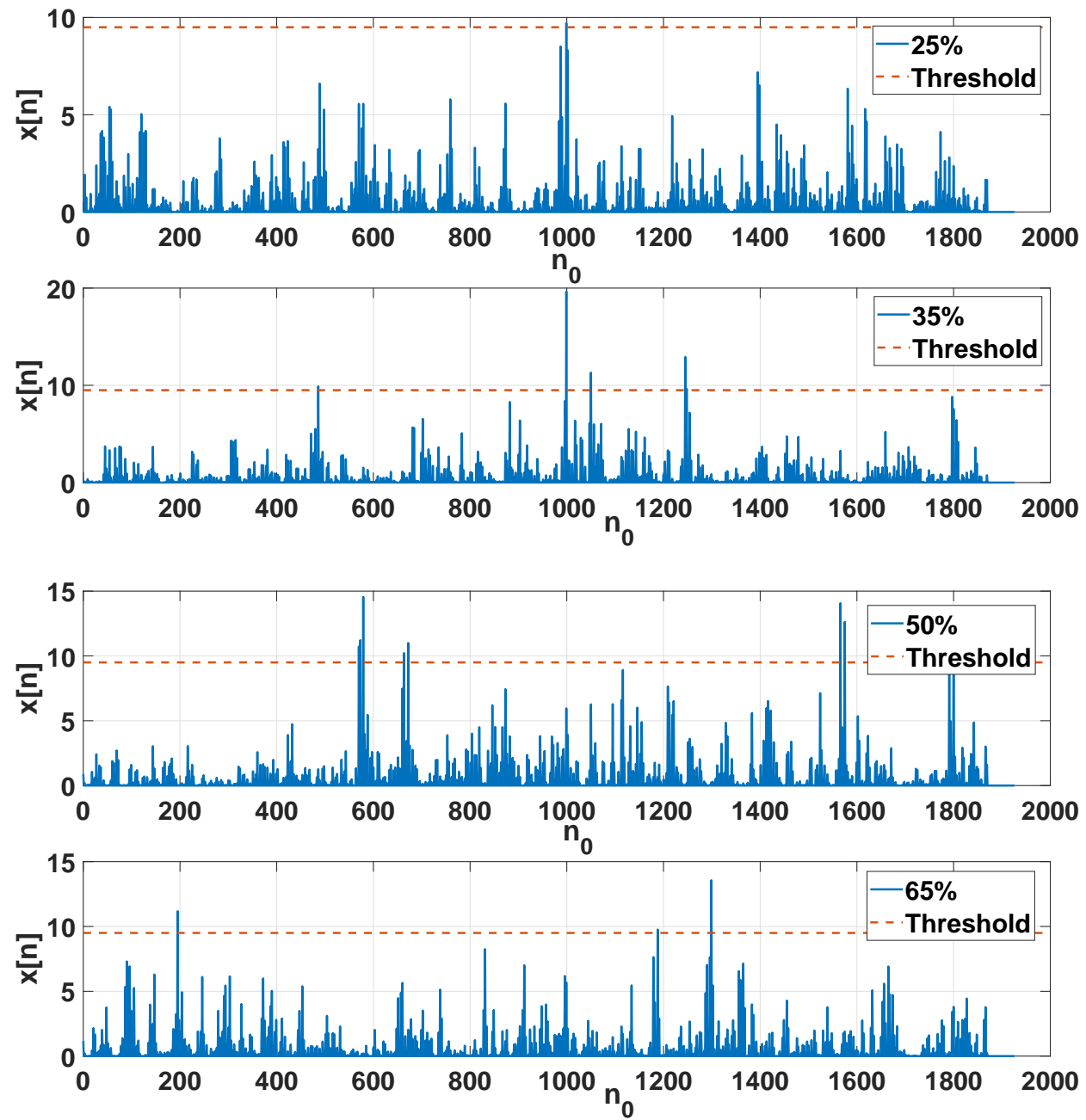

Figure 29: Test statistic $T_{n_{0}}(\mathbf{x})$ vs $n_{0}$ using GAMS signal (GLRT Implementation)

It was shown that the optimal signal is an impulse and as a result the signal has an impulsive ACS. Since an impulsive ACS signal is not realizable in practice alternatives have been studied. The different signals fall into four different categories, which we called LFM, NLFM, PCM, and other signals. It was shown that all suggested signals have an advantage of almost $10 \mathrm{~dB}$ in compare to the most commonly used probe excitation signal GAMS signal, which has a narrowband spectrum. Signals that we called Chu, Golomb, CAN, and MISL have a small advantage over the LFM and NLFM signals. Still an LFM signal in terms of practical implementation has an advantage in comparison to the others. Also, it was 
shown that by using any of the suggested signals an almost optimal performance in terms of detectability can be achieved. For future work since different applications are concerned with different properties of the signal a more extensive study and comparison among these signals will be pursued. Moreover, a collaboration with other researchers is ongoing that will focus on the experimental verification of these results and a deeper look at the advantages and disadvantages of using each of these signals for different applications. 


\section{Appendix 2A. Optimal Signal Proof for Finite Data Record}

We wish to maximize the deflection coefficient

$$
d^{2}=\mathbf{s}^{T}\left(\mathbf{C}_{c}+\sigma_{w}^{2} \mathbf{I}\right)^{-1} \mathbf{s}
$$

where $\mathbf{s}=[s[0] s[1] \ldots s[N-1]]^{T}$ is the signal, $\mathbf{C}_{c}$ is the clutter covariance matrix, and $\sigma_{w}^{2}$ is the white Gaussian noise variance. The clutter covariance matrix is given by (4)

$$
\left[\mathbf{C}_{c}\right]_{i j}=\alpha \sum_{n=0}^{N-|i-j|} s[n] s[n+|i-j|]=r_{c}[i-j]
$$

for $i=1,2, \ldots, N, j=1,2, \ldots, N$ and is a symmetric Toeplitz autocorrelation matrix with $[i, j]$ element the scaled by $\alpha$ autocorrelation sequence of the signal and denoted by $r_{c}[i-j]$. The maximization is under the constraint that the signal energy is fixed, which is $\mathbf{s}^{T} \mathbf{s}=\mathcal{E}$. Otherwise the maximum is obtained by letting the signal energy go to infinity. Now when we need to illustrate the various quantities we will use $N=3$, but the proof is valid for general $N$. 
The proof proceeds as follows. Letting $N=3$ for illustration we note that

$$
\begin{aligned}
& \mathbf{C}_{c}=\left[\begin{array}{lll}
r_{c}[0] & r_{c}[1] & r_{c}[2] \\
r_{c}[1] & r_{c}[0] & r_{c}[1] \\
r_{c}[2] & r_{c}[1] & r_{c}[0]
\end{array}\right]
\end{aligned}
$$

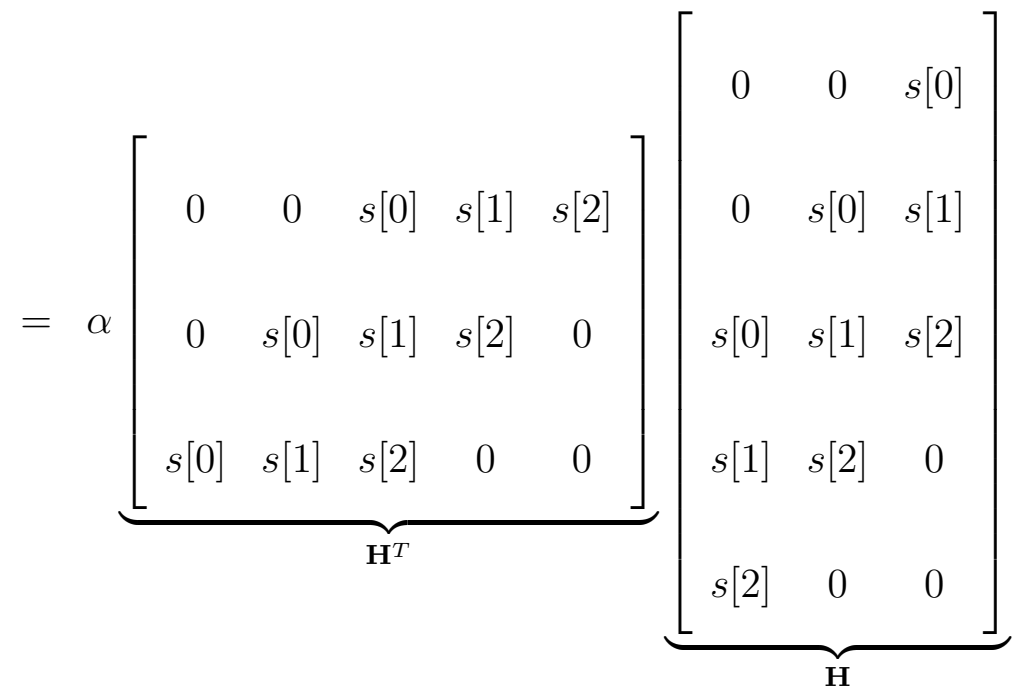

Note that $\mathbf{H}$ is $(2 N-1) \times N=5 \times 3$ and that $\mathbf{C}_{c}=\alpha \mathbf{H}^{T} \mathbf{H}$. Also, the middle column of $\mathbf{H}^{T}$ is $\mathbf{s}$, which may be expressed for this example as $\mathbf{H}^{T} \mathbf{e}_{3}=\mathbf{s}$, where $\mathbf{e}_{3}$ is the $2 N-1 \times 1$ natural unit vector with a one at its middle element and zero otherwise. In this case $\mathbf{e}_{3}=\left[\begin{array}{lllll}0 & 0 & 1 & 0 & 0\end{array}\right]^{T}$. Now we wish to maximize

$$
\begin{aligned}
d^{2} & =\mathbf{s}^{T}\left(\mathbf{C}_{c}+\sigma_{w}^{2} \mathbf{I}\right)^{-1} \mathbf{s} \\
& =\left(\mathbf{H}^{T} \mathbf{e}_{N}\right)^{T}\left(\mathbf{C}_{c}+\sigma_{w}^{2} \mathbf{I}\right)^{-1} \mathbf{H}^{T} \mathbf{e}_{N} \\
& =\mathbf{e}_{N}^{T} \mathbf{H}\left(\alpha \mathbf{H}^{T} \mathbf{H}+\sigma_{w}^{2} \mathbf{I}\right)^{-1} \mathbf{H}^{T} \mathbf{e}_{N} \\
& =\frac{1}{\alpha} \mathbf{e}_{N}^{T} \mathbf{H}\left(\mathbf{H}^{T} \mathbf{H}+\left(\sigma_{w}^{2} / \alpha\right) \mathbf{I}\right)^{-1} \mathbf{H}^{T} \mathbf{e}_{N}
\end{aligned}
$$

We next use a singular value decomposition (SVD) to diagonalize $\mathbf{H}$ as

$$
\underbrace{\mathbf{U}^{T}}_{5 \times 5} \underbrace{\mathbf{H}}_{5 \times 3} \underbrace{\mathbf{V}}_{3 \times 3}=\underbrace{\mathbf{\Sigma}}_{5 \times 3}
$$


where $\mathbf{U}^{T} \mathbf{U}=\mathbf{I}_{5}, \mathbf{V}^{T} \mathbf{V}=\mathbf{I}_{3}$, and

$$
\begin{gathered}
\boldsymbol{\Sigma}=\left[\begin{array}{l}
\boldsymbol{\xi} \\
\mathbf{0}
\end{array}\right]=\left[\begin{array}{l}
3 \times 3 \\
2 \times 3
\end{array}\right] . \\
\boldsymbol{\xi}=\left[\begin{array}{lll}
\xi_{1} & 0 & 0 \\
0 & \xi_{2} & 0 \\
0 & 0 & \xi_{3}
\end{array}\right] .
\end{gathered}
$$

Here $\xi_{i}$ are the singular values with $\xi_{i} \geq 0$ and $\mathbf{U}=\left[\mathbf{u}_{1} \ldots \mathbf{u}_{5}\right], \mathbf{V}=\left[\mathbf{v}_{1} \mathbf{v}_{2} \mathbf{v}_{3}\right]$ are the matrices whose columns span the range spaces of $\mathbf{H}$ and $\mathbf{H}^{T}$, respectively. Note that the columns of each matrix are orthonormal. Finally, $\lambda_{i}=\xi_{i}^{2}$ are the eigenvalues of $\mathbf{H}^{T} \mathbf{H}$ or equivalently $\boldsymbol{\xi}^{T} \boldsymbol{\xi}=\boldsymbol{\Lambda}$, where $\mathbf{V}^{T} \mathbf{H}^{T} \mathbf{H V}=\boldsymbol{\Lambda}$. Now using $\mathbf{H}=\mathbf{U} \boldsymbol{\Sigma} \mathbf{V}^{T}$ we have

$$
\begin{aligned}
d^{2} & =\frac{1}{\alpha} \mathbf{e}_{N}^{T} \mathbf{U} \boldsymbol{\Sigma} \mathbf{V}^{T}\left(\mathbf{V} \boldsymbol{\Sigma}^{T} \boldsymbol{\Sigma} \mathbf{V}^{T}+\left(\sigma_{w}^{2} / \alpha\right) \mathbf{I}\right)^{-1} \mathbf{V} \boldsymbol{\Sigma}^{T} \mathbf{U}^{T} \mathbf{e}_{N} \\
& =\frac{1}{\alpha} \mathbf{e}_{N}^{T} \mathbf{U} \boldsymbol{\Sigma}\left[\mathbf{V}^{-1}\left(\mathbf{V} \boldsymbol{\Sigma}^{T} \boldsymbol{\Sigma} \mathbf{V}^{T}+\left(\sigma_{w}^{2} / \alpha\right) \mathbf{I}\right) \mathbf{V}\right]^{-1} \boldsymbol{\Sigma}^{T} \mathbf{U}^{T} \mathbf{e}_{N} \\
& =\frac{1}{\alpha} \mathbf{e}_{N}^{T} \mathbf{U} \underbrace{\boldsymbol{\Sigma}\left[\boldsymbol{\Sigma}^{T} \boldsymbol{\Sigma}+\left(\sigma_{w}^{2} / \alpha\right) \mathbf{I}\right]^{-1} \boldsymbol{\Sigma}^{T}}_{\mathbf{A}} \mathbf{U}^{T} \mathbf{e}_{N}
\end{aligned}
$$

and note that $\boldsymbol{\Sigma}^{T} \boldsymbol{\Sigma}=\boldsymbol{\Lambda}$. Hence, we have that

$$
\begin{aligned}
\mathbf{A} & =\left[\begin{array}{l}
\boldsymbol{\xi} \\
\mathbf{0}
\end{array}\right]\left(\boldsymbol{\Lambda}+\left(\sigma_{w}^{2} / \alpha\right) \mathbf{I}\right)^{-1}\left[\begin{array}{ll}
\boldsymbol{\xi} & 0
\end{array}\right] \\
& =\left[\begin{array}{cc}
\boldsymbol{\xi}\left(\boldsymbol{\Lambda}+\left(\sigma_{w}^{2} / \alpha\right) \mathbf{I}\right)^{-1} \boldsymbol{\xi} & \mathbf{0} \\
\mathbf{0} & \mathbf{0}
\end{array}\right]
\end{aligned}
$$


which for $N=3$ becomes

$$
\mathbf{A}=\left[\begin{array}{cccccc}
\frac{\lambda_{1}}{\lambda_{1}+\left(\sigma_{w}^{2} / \alpha\right)} & 0 & 0 & \mid & 0 & 0 \\
0 & \frac{\lambda_{2}}{\lambda_{2}+\left(\sigma_{w}^{2} / \alpha\right)} & 0 & \mid & 0 & 0 \\
0 & 0 & \frac{\lambda_{3}}{\lambda_{3}+\left(\sigma_{w}^{2} / \alpha\right)} & \mid & 0 & 0 \\
- & - & - & - & - & - \\
0 & 0 & 0 & \mid & 0 & 0 \\
0 & 0 & 0 & \mid & 0 & 0
\end{array}\right] .
$$

With this diagonalization, we can write

$$
\begin{aligned}
d^{2} & =\frac{1}{\alpha} \mathbf{e}_{N}^{T} \mathbf{U} \mathbf{A} \mathbf{U}^{T} \mathbf{e}_{N} \\
& =\frac{1}{\alpha} \mathbf{e}_{N}^{T} \sum_{i=1}^{N} \frac{\lambda_{i}}{\lambda_{i}+\left(\sigma_{w}^{2} / \alpha\right)} \mathbf{u}_{i} \mathbf{u}_{i}^{T} \mathbf{e}_{N} \\
& =\frac{1}{\alpha} \sum_{i=1}^{N} \underbrace{\left(\mathbf{u}_{i}^{T} \mathbf{e}_{N}\right)^{2}}_{\eta_{i}} \frac{\lambda_{i}}{\lambda_{i}+\left(\sigma_{w}^{2} / \alpha\right)} .
\end{aligned}
$$

Now since the $\lambda_{i}$ are the eigenvalues of $\mathbf{H}^{T} \mathbf{H}$, which is assumed positive semidefinite, they are nonnegative and satisfy

$$
\sum_{i=1}^{N} \lambda_{i}=\operatorname{tr}\left(\mathbf{H}^{T} \mathbf{H}\right)=N \mathbf{s}^{T} \mathbf{s}=N \mathcal{E}
$$

As a result, we need to maximize the function

$$
g(\boldsymbol{\lambda}, \boldsymbol{\eta})=\sum_{i=1}^{N} \eta_{i} \frac{\lambda_{i}}{\lambda_{i}+\beta}
$$

over $\boldsymbol{\lambda}$ subject to the constraints that $\lambda_{i} \geq 0, \sum_{i=1}^{N} \lambda_{i}=N \mathcal{E}$, and over $\boldsymbol{\eta}$ subject to the constraint that $\eta_{i} \geq 0$ and also $\sum_{i=1}^{N} \eta_{i} \leq 1$. We have also let $\beta=\left(\sigma_{w}^{2} / \alpha\right)>0$. 
It is clear that $\eta_{i}=\left(\mathbf{u}_{i}^{T} \mathbf{e}_{N}\right)^{2} \geq 0$. To verify the last constraint note that

$$
\begin{aligned}
\sum_{i=1}^{N} \eta_{i} & =\sum_{i=1}^{N} \mathbf{e}_{N}^{T} \mathbf{u}_{i} \mathbf{u}_{i}^{T} \mathbf{e}_{N} \\
& =\mathbf{e}_{N}^{T} \underbrace{\sum_{i=1}^{N} \mathbf{u}_{i} \mathbf{u}_{i}^{T}}_{\mathbf{P}_{H}} \mathbf{e}_{N} \\
& =\mathbf{e}_{N}^{T} \mathbf{P}_{H} \mathbf{e}_{N} \\
& =\left\|\mathbf{P}_{H} \mathbf{e}_{N}\right\|^{2}
\end{aligned}
$$

where $\mathbf{P}_{H}$ is the projection matrix onto the columns of $\mathbf{H}$, and hence

$$
\sum_{i=1}^{N} \eta_{i}=\left\|\mathbf{P}_{H} \mathbf{e}_{N}\right\|^{2} \leq\left\|\mathbf{e}_{N}\right\|^{2}=1 .
$$

We can let the constraint be an equality since clearly $d^{2}$ is monotonically increasing with a scaling of all the $\eta_{i}$ 's. Thus, the maximum over $\boldsymbol{\eta}$ must occur when $\sum_{i=1}^{N} \eta_{i}=$ 1.

To summarize we wish to determine the maximum of

$$
g(\boldsymbol{\lambda}, \boldsymbol{\eta})=\sum_{i=1}^{N} \eta_{i} \frac{\lambda_{i}}{\lambda_{i}+\beta}
$$

subject to the equality constraints $\sum_{i=1}^{N} \lambda_{i}=N \mathcal{E}$ and $\sum_{i=1}^{N} \eta_{i}=1$. To do so we use Lagrangian multipliers, ignoring the positivity constraints of $\lambda_{i}$ and $\eta_{i}$. The Lagrangian is

$$
\begin{aligned}
\mathcal{L}(\boldsymbol{\lambda}, \boldsymbol{\eta}) & =\sum_{i=1}^{N} \eta_{i} \frac{\lambda_{i}}{\lambda_{i}+\beta}-k_{1}\left(\sum_{i=1}^{N} \lambda_{i}-\mathcal{E}\right)-k_{2}\left(\sum_{i=1}^{N} \eta_{i}-1\right) \\
& =\sum_{i=1}^{N}\left(\eta_{i} \frac{\lambda_{i}}{\lambda_{i}+\beta}-k_{1} \lambda_{i}-k_{2} \eta_{i}\right)+k_{1} \mathcal{E}+k_{2} .
\end{aligned}
$$

Here of course $k_{1}, k_{2}$ are the Lagrangian multipliers. Now the term in parenthesis is the function

$$
f(\lambda, \eta)=\eta \frac{\lambda}{\lambda+\beta}-k_{1} \lambda-k_{2} \eta
$$


which is easily shown to be concave for $\lambda \geq 0$ and $\eta \geq 0$ and so the overall function $g$ is also concave. We can maximize $\mathcal{L}(\boldsymbol{\lambda}, \boldsymbol{\eta})$ by maximizing each individual term and note that it can be done by differentiation due to the concavity. Now

$$
\begin{aligned}
& \frac{\partial f}{\partial \lambda}=\eta\left[\frac{\lambda+\beta-\lambda}{(\lambda+\beta)^{2}}\right]-k_{1}=0 \\
& \frac{\partial f}{\partial \eta}=\frac{\lambda}{\lambda+\beta}-k_{2}=0
\end{aligned}
$$

from which

$$
\begin{aligned}
\frac{\eta \beta}{(\lambda+\beta)^{2}} & =k_{1} \\
\frac{\lambda}{\lambda+\beta} & =k_{2} .
\end{aligned}
$$

Solving for $\lambda$ and $\eta$, we have from the second equation

$$
\tilde{\lambda}=\frac{k_{2} \beta}{1-k_{2}}
$$

and inserting into the first equation

$$
\begin{aligned}
\tilde{\eta} & =\frac{k_{1}}{\beta}(\lambda+\beta)^{2} \\
& =\frac{k_{1}}{\beta}\left(\frac{k_{2} \beta}{1-k_{2}}+\beta\right)^{2} \\
& =\frac{\beta k_{1}}{\left(1-k_{2}\right)^{2}} .
\end{aligned}
$$

To find $k_{1}, k_{2}$, we note that $\tilde{\lambda}$ and $\tilde{\eta}$ do not depend on $i$, and hence, we use the constraints to yield

$$
\sum_{i=1}^{N} \tilde{\lambda}_{i}=\frac{N \beta k_{2}}{1-k_{2}}=N \mathcal{E}
$$

which is easily shown to be

$$
\tilde{k}_{2}=\frac{\mathcal{E}}{\mathcal{E}+\beta}
$$

Thus, from (23)

$$
\sum_{i=1}^{N} \tilde{\eta}_{i}=\frac{N \beta k_{1}}{\left(1-k_{2}\right)^{2}}=1
$$


which after some manipulation yields

$$
\tilde{k}_{1}=\frac{\beta}{N(\mathcal{E}+\beta)^{2}}
$$

Finally, substituting the values of the Lagrangian multipliers into (22) and (23) we arrive at the maximizing solutions

$$
\begin{aligned}
\tilde{\lambda}_{i} & =\mathcal{E} \\
\tilde{\eta}_{i} & =\frac{1}{N} .
\end{aligned}
$$

The maximum is therefore

$$
d_{\max }^{2}=\frac{1}{\alpha} \sum_{i=1}^{N} \eta_{i} \frac{\lambda_{i}}{\lambda_{i}+\left(\sigma_{w}^{2} / \alpha\right)}=\frac{1}{\alpha} \frac{\mathcal{E}}{\mathcal{E}+\frac{\sigma_{w}^{2}}{\alpha}}=\frac{\mathcal{E}}{\alpha \mathcal{E}+\sigma_{w}^{2}} .
$$

Next we need to determine the signal s so that this maximum is realized. First, since $\tilde{\lambda}_{i}=\mathcal{E}$ and recall that $\lambda_{i}$ is the $i$ th eigenvalue of $\mathbf{H}^{T} \mathbf{H}$, we must have that using $\mathbf{V}^{T} \mathbf{H}^{T} \mathbf{H V}=\boldsymbol{\Lambda}$

$$
\begin{aligned}
\mathbf{C}_{c} & =\alpha \mathbf{H}^{T} \mathbf{H} \\
& =\alpha \mathbf{V} \boldsymbol{\Lambda} \mathbf{V}^{T} \\
& =\alpha \mathbf{V} \mathcal{E} \mathbf{I} \mathbf{V}^{T} \\
& =\alpha \mathcal{E} \mathbf{I} .
\end{aligned}
$$

Hence, the autocorrelation sequence must be impulsive or $r_{c}[k]=0$ for $k \neq 0$, and $r_{c}[0]=\alpha \mathcal{E}$. Secondly, we must find a finite signal sequence that satisfies $\eta_{i}=\left(\mathbf{u}_{i}^{T} \mathbf{e}_{N}\right)^{2}=1 / N$ for $i=1,2, \ldots, N$ in order to satisfy the second constraint. Using $N=3$ to illustrate, we require $\left(\mathbf{e}_{3}^{T} \mathbf{u}_{i}\right)^{2}=1 / 3$ for $i=1,2,3$ (recall that $\mathbf{e}_{N}$ and $\mathbf{u}_{i}$ are $\left.(2 N-1) \times 1=5 \times 1\right)$. Noting that

$$
\mathbf{P}_{H}=\mathbf{u}_{1} \mathbf{u}_{1}^{T}+\mathbf{u}_{2} \mathbf{u}_{2}^{T}+\mathbf{u}_{3} \mathbf{u}_{3}^{T}
$$


is the projection matrix onto the columns of $\mathbf{H}$, we have that $\mathbf{e}_{3}=\mathbf{P}_{H} \mathbf{e}_{3}+\mathbf{P}_{H}^{\perp} \mathbf{e}_{3}$, where $\mathbf{P}_{H}^{\perp}$ is the projection matrix onto the orthogonal subspace of $\mathbf{H}$. Now

$$
\begin{aligned}
\left\|\mathbf{e}_{3}\right\|^{2} & =\left\|\mathbf{P}_{H} \mathbf{e}_{3}\right\|^{2}+\left\|\mathbf{P}_{H}^{\perp} \mathbf{e}_{3}\right\|^{2} \\
& =\left\|\left(\mathbf{u}_{1} \mathbf{u}_{1}^{T}+\mathbf{u}_{2} \mathbf{u}_{2}^{T}+\mathbf{u}_{3} \mathbf{u}_{3}^{T}\right) \mathbf{e}_{3}\right\|^{2}+\left\|\mathbf{P}_{H}^{\perp} \mathbf{e}_{3}\right\|^{2} \\
& =\left(\mathbf{u}_{1}^{T} \mathbf{e}_{3}\right)^{2}+\left(\mathbf{u}_{2}^{T} \mathbf{e}_{3}\right)^{2}+\left(\mathbf{u}_{3}^{T} \mathbf{e}_{3}\right)^{2}+\left\|\mathbf{P}_{H}^{\perp} \mathbf{e}_{3}\right\|^{2} \\
& =\eta_{1}+\eta_{2}+\eta_{3}+\left\|\mathbf{P}_{H}^{\perp} \mathbf{e}_{3}\right\|^{2} \\
& =\frac{1}{3}+\frac{1}{3}+\frac{1}{3}+\left\|\mathbf{P}_{H}^{\perp} \mathbf{e}_{3}\right\|^{2} \\
& =1+\left\|\mathbf{P}_{H}^{\perp} \mathbf{e}_{3}\right\|^{2} .
\end{aligned}
$$

But since $\left\|\mathbf{e}_{3}\right\|^{2}=1$, we have that $\left\|\mathbf{P}_{H}^{\perp} \mathbf{e}_{3}\right\|^{2}=0$ and therefore $\mathbf{P}_{H}^{\perp} \mathbf{e}_{3}=\mathbf{0}$. This says that $\mathbf{e}_{3}$ must lie in the column subspace of $\mathbf{H}$ or for this illustration it must be true that

$$
\mathbf{e}_{3}=c_{1} \mathbf{h}_{1}+c_{2} \mathbf{h}_{2}+c_{3} \mathbf{h}_{3}
$$

for some constants $c_{i}$. Letting $c_{1}=h[0], c_{2}=h[-1]$ and $c_{3}=h[-2]$, we then have the equivalent condition

$$
\underbrace{\left[\begin{array}{ccc}
0 & 0 & s[0] \\
0 & s[0] & s[1] \\
s[0] & s[1] & s[2] \\
s[1] & s[2] & 0 \\
s[2] & 0 & 0
\end{array}\right]}_{\mathbf{H}} h\left[\begin{array}{c}
h[0] \\
h[-2]
\end{array}\right]=\underbrace{\left[\begin{array}{c}
0 \\
1 \\
0 \\
0
\end{array}\right]}_{\mathbf{e}_{3}} .
$$

This may be recognized as a convolution-type relationship or $s[n] * h[n]=\delta[n]$, where $\delta[n]$ is a discrete-time impulse. It also holds for all $n$ if we were to extend 
the number of rows in $\mathbf{H}$ "in both directions" using zeros as elements. Therefore, taking the Fourier transform of the convolution we have that

$$
S(f) H(f)=1
$$

or

$$
S(f)=\frac{1}{H(f)}=\frac{1}{h[0]+h[-1] \exp (j 2 \pi f)+h[-2] \exp (j 4 \pi f)} .
$$

But since $s[n]$ is constrained to be a finite length causal sequence, its z-transform is (with a slight abuse of notation)

$$
S(z)=\frac{1}{h[0]+h[-1] z+h[-2] z^{2}} .
$$

To invert this transform and find $s[n]$, we need to look at the more general case of $N$. This is because any of the $h[-n]$ 's may be zero, affecting the inverse transform. Hence, consider

$$
S(z)=\frac{1}{h[0]+h[-1] z+h[-2] z^{2}+\cdots+h[-(N-1)] z^{N-1}}
$$

and assume without loss of generality that the minimum power of $z$ for which $h[-n] \neq 0$ is $k$, i.e., $z^{k}$. Also, the maximum power of $z$ for which $h[-n] \neq 0$ is $l$, i.e., $z^{l}$. Clearly, we can have $k=0,1, \ldots, N-1$ and $l \geq k$. Then,

$$
S(z)=\frac{1}{h[-k] z^{k}+\cdots+h[-l] z^{l}} .
$$

Now if $l=k$ we must have

$$
S(z)=\frac{1}{h[-k] z^{k}}
$$

for which $s[n]=(1 / h[-k]) \delta[n-k]$ for any $k=0,1, \ldots, N-1$. Otherwise, for $l$ strictly greater than $k$ and hence at least two different powers of $z$ in the denominator, we have

$$
\begin{aligned}
S(z) & =\frac{1}{h[-l] z^{l}\left(1+\frac{h[-(l-1)]}{h[-l]} z^{-1}+\frac{h[-(l-2)]}{h[-l]} z^{-2}+\cdots+\frac{h[-k]}{h[-l]} z^{-(l-k)}\right)} \\
& =\frac{\frac{1}{h[-l]} z^{-l}}{1+\frac{h[-(l-1)]}{h[-l]} z^{-1}+\frac{h[-(l-2)]}{h[-l]} z^{-2}+\cdots+\frac{h[-k]}{h[-l]} z^{-(l-k)}} .
\end{aligned}
$$


For this to be the $z$-transform of a finite length sequence, the denominator polynomial must be a constant or we require $h[-(l-1)]=h[-(l-2)]=\cdots=h[-k]=0$. Thus, we must have

$$
S(z)=\frac{1}{h[-l]} z^{-l}
$$

and therefore

$$
s[n]=\frac{1}{h[-l]} \delta[n-l]=\sqrt{\mathcal{E}} \delta[n-l]
$$

for any $l=0,1, \ldots, N-1$, which is the same as before. Note also that these signals satisfy the other constraint that the autocorrelation sequence must be impulsive. The maximum SNR is $d_{M A X}^{2}=\mathbf{s}^{T}\left(\mathbf{C}_{c}+\sigma_{w}^{2} \mathbf{I}\right)^{-1} \mathbf{s}=\mathbf{s}^{T}\left(\alpha \mathcal{E} \mathbf{I}+\sigma_{w}^{2} \mathbf{I}\right)^{-1} \mathbf{s}=\frac{\mathcal{E}}{\alpha \mathcal{E}+\sigma_{w}^{2}}$.

\section{List of References}

[1] J. Thompson and E. Titlebaum, "The design of optimal radar waveforms for clutter rejection using the maximum principle." 1967.

[2] D. DeLong and E. Hofstetter, "The design of clutter-resistant radar waveforms with limited dynamic range," IEEE Transactions on Information Theory, vol. 15, pp. 376-385, 1969.

[3] P. Burrascano, S. Laureti, L. Senni, and M. Ricci, "Pulse compression in nondestructive testing applications: reduction of near sidelobes exploiting reactance transformation," IEEE Transactions on Circuits and Systems I: Regular Papers, vol. 66, pp. 1886-1896, 2018.

[4] S. M. Kay, Fundamentals of Statistical Signal Processing: Detection. Prentice Hall PTR, 1998.

[5] Y. Rawashdeh and S. Kay, "A new physically motivated clutter model with applications to non-destructive ultrasonic testing," IEEE Transactions on Ultrasonics, Ferroelectrics, and Frequency Control, 2020.

[6] T. Niederdränk, "Maximum length sequences in non-destructive material testing: application of piezoelectric transducers and effects of time variances," Ultrasonics, vol. 35, pp. 195-203, 1997.

[7] M. Ricci, S. Callegari, S. Caporale, M. Monticelli, L. Battaglini, M. Eroli, L. Senni, R. Rovatti, G. Setti, and P. Burrascano, "Exploiting non-linear chirp and sparse deconvolution to enhance the performance of pulse-compression ultrasonic ndt," in 2012 IEEE International Ultrasonics Symposium. IEEE, 2012, pp. 1489-1492. 
[8] S. Laureti, S. Sfarra, H. Malekmohammadi, P. Burrascano, D. A. Hutchins, L. Senni, G. Silipigni, X. Maldague, and M. Ricci, "The use of pulsecompression thermography for detecting defects in paintings," Ndt $\&$ E International, vol. 98, pp. 147-154, 2018.

[9] S. Callegari, M. Ricci, S. Caporale, M. Monticelli, M. Eroli, L. Senni, R. Rovatti, G. Setti, and P. Burrascano, "From chirps to random-fm excitations in pulse compression ultrasound systems," in 2012 IEEE International Ultrasonics Symposium. IEEE, 2012, pp. 471-474.

[10] M. Pollakowski and H. Ermert, "Chirp signal matching and signal power optimization in pulse-echo mode ultrasonic nondestructive testing," IEEE transactions on ultrasonics, ferroelectrics, and frequency control, vol. 41, pp. 655-659, 1994.

[11] S. Kay, Intuitive probability and random processes using MATLAB囚. Springer Science \& Business Media, 2006.

[12] H. L. Van Trees and K. L. Bell, Detection estimation and modulation theory. John Wiley \& Sons, 2004.

[13] M. I. Skolnik, RADAR systems. McGraw-Hill, NY, 2001.

[14] H. He, J. Li, and P. Stoica, Waveform design for active sensing systems: a computational approach. Cambridge University Press, 2012.

[15] A. Misaridis, "Ultrasound imaging using coded signals," 2001.

[16] N. Levanon and E. Mozeson, Radar signals. John Wiley \& Sons, 2004.

[17] S. Alphonse and G. A. Williamson, "Evaluation of a class of nlfm radar signals," EURASIP Journal on Advances in Signal Processing, vol. 2019, p. 62, 2019.

[18] S. Boukeffa, Y. Jiang, and T. Jiang, "Sidelobe reduction with nonlinear frequency modulated waveforms," in 2011 IEEE rth International Colloquium on Signal Processing and its Applications. IEEE, 2011, pp. 399-403.

[19] R. Price, "Chebyshev low pulse compression sidelobes via a nonlinear fm," in National Radio Science Meeting of URSI, 1979.

[20] C. Cook, "A class of nonlinear fm pulse compression signals," Proceedings of the IEEE, vol. 52, pp. 1369-1371, 1964.

[21] D. Chu, "Polyphase codes with good periodic correlation properties (corresp.)," IEEE Transactions on information theory, vol. 18, no. 4, pp. 531-532, 1972. 
[22] N. Zhang and S. W. Golomb, "Polyphase sequence with low autocorrelations," IEEE Transactions on Information Theory, vol. 39, pp. 1085-1089, 1993.

[23] P. Stoica, R. L. Moses, et al., "Spectral analysis of signals," 2005.

[24] P. Stoica, H. He, and J. Li, "New algorithms for designing unimodular sequences with good correlation properties," IEEE Transactions on Signal Processing, vol. 57, no. 4, pp. 1415-1425, 2009.

[25] J. Song, P. Babu, and D. P. Palomar, "Optimization methods for designing sequences with low autocorrelation sidelobes," IEEE Transactions on Signal Processing, vol. 63, pp. 3998-4009, 2015.

[26] D. R. Hunter and K. Lange, "A tutorial on mm algorithms," The American Statistician, vol. 58, pp. 30-37, 2004.

[27] P. Stoica and Y. Selen, "Cyclic minimizers, majorization techniques, and the expectation-maximization algorithm: a refresher," IEEE Signal Processing Magazine, vol. 21, no. 1, pp. 112-114, 2004.

[28] C.-P. Chiou, R. B. Thompson, and L. W. Schmerr, "Model-based signal processing techniques for ultrasonic flaw detection: Simulation studies," in Review of Progress in Quantitative Nondestructive Evaluation. Springer, 1993, pp. 703-710.

[29] M. G. Gustafsson, "Nonlinear clutter suppression using split spectrum processing and optimal detection," IEEE transactions on ultrasonics, ferroelectrics, and frequency control, vol. 43, pp. 109-124, 1996.

[30] I. Yalda-Mooshabad, F. J. Margetan, and R. B. Thompson, "Monte-carlo simulation of ultrasonic grain noise," in Review of progress in Quantitative Nondestructive Evaluation. Springer, 1993, pp. 1727-1734.

[31] S. Chatillon, C. Poidevin, N. Gengembre, and A. Lhemery, "Simplified modeling of backscattered noise and attenuation phenomena for quantitative performance demonstration of ut methods," in AIP Conference Proceedings. IOP INSTITUTE OF PHYSICS PUBLISHING LTD, 2003, pp. 93-100.

[32] V. DORVAL, L. DUCOUSSO-GANJEHI, S. CHATILLON, and F. JENSON, "Modeling ultrasonic structural noise based on the microstructural properties of metals in the context of non destructive evaluation," in 13th International Symposium on Nondestructive Characterization of Materials (NDCM-XIII), 2013, pp. 20-24.

[33] K. D. Donohue, "Maximum likelihood estimation of a-scan amplitudes for coherent targets in media of unresolvable scatterers," IEEE transactions on ultrasonics, ferroelectrics, and frequency control, vol. 39.3, pp. 422-431, 1992. 


\section{CHAPTER 3}

\section{Future Work}

The work that was presented in this thesis can be extended to accommodate wider range of problems:

- Our physically motivated model showed robustness and excellent results when applied on real ultrasonic data, which makes the possibility of extending the model to the two-dimensional case worth pursuing, hence, extend the model to more valuable applications such as medical imaging.

- A complete implementation of the probabilistically motivated model using Poisson filtered process to be completed. Interesting enough, the model led to the same result as the physically motivated one, where the clutter noise has a flat spectrum that is a scaled version of the ESD of the transmitted signal. However, the probabilistically motivated model result in more return pulses for the same scanned volume of the material in compare to the physically motivated model, which can be utilized to accommodate a wider range of materials with different homogeneity and isotropy levels.

- In regards to the optimal signal design a full experimental verification for the theocratical and simulated results should be completed. A comparison analysis between the proposed signals for different applications to point out the advantages and disadvantages of each can be pursued.

- Applying the physically motivated model on more sample for different materials and defects to understand the strength and limitations of the model. 


\section{BIBLIOGRAPHY}

Alphonse, S. and Williamson, G. A., "Evaluation of a class of nlfm radar signals," EURASIP Journal on Advances in Signal Processing, vol. 2019, p. 62, 2019.

Bell, M. R., "Information theory and radar waveform design," IEEE Transactions on Information Theory, vol. 39, no. 5, pp. 1578-1597, 1993.

Blitz, J. and Simpson, G., Ultrasonic methods of non-destructive testing. Vol. 2. Springer Science \& Business Media, 1995.

Boukeffa, S., Jiang, Y., and Jiang, T., "Sidelobe reduction with nonlinear frequency modulated waveforms," in 2011 IEEE 7th International Colloquium on Signal Processing and its Applications. IEEE, 2011, pp. 399-403.

Burrascano, P., Laureti, S., Senni, L., and Ricci, M., "Pulse compression in nondestructive testing applications: reduction of near sidelobes exploiting reactance transformation," IEEE Transactions on Circuits and Systems I: Regular Papers, vol. 66, pp. 1886-1896, 2018.

Callegari, S., Ricci, M., Caporale, S., Monticelli, M., Eroli, M., Senni, L., Rovatti, R., Setti, G., and Burrascano, P., "From chirps to random-fm excitations in pulse compression ultrasound systems," in 2012 IEEE International Ultrasonics Symposium. IEEE, 2012, pp. 471-474.

Charlesworth, J. P. and Temple, J. A. G., Engineering applications of ultrasonic time-of-flight diffraction. Vol. 1. John Wiley \& Sons Inc, 1989.

Chatillon, S., Poidevin, C., Gengembre, N., and Lhemery, A., "Simplified modeling of backscattered noise and attenuation phenomena for quantitative performance demonstration of ut methods," in AIP Conference Proceedings. IOP INSTITUTE OF PHYSICS PUBLISHING LTD, 2003, pp. 93-100.

Chiou, C.-P., Thompson, R. B., and Schmerr, L. W., "Model-based signal processing techniques for ultrasonic flaw detection: Simulation studies," in Review of Progress in Quantitative Nondestructive Evaluation. Springer, 1993, pp. 703-710.

Chu, D., "Polyphase codes with good periodic correlation properties (corresp.)," IEEE Transactions on information theory, vol. 18, no. 4, pp. 531-532, 1972.

Cohen, F. S., "Modeling of ultrasound speckle with application in flaw detection in metals," IEEE Transactions on signal processing, vol. 40, pp. 624-632, 1992.

Cook, C., "A class of nonlinear fm pulse compression signals," Proceedings of the IEEE, vol. 52, pp. 1369-1371, 1964. 
DeLong, D. and Hofstetter, E., "The design of clutter-resistant radar waveforms with limited dynamic range," IEEE Transactions on Information Theory, vol. 15, pp. 376-385, 1969.

Donohue, K. D., "Maximum likelihood estimation of a-scan amplitudes for coherent targets in media of unresolvable scatterers," IEEE transactions on ultrasonics, ferroelectrics, and frequency control, vol. 39.3, pp. 422-431, 1992.

DORVAL, V., DUCOUSSO-GANJEHI, L., CHATILLON, S., and JENSON, F., "Modeling ultrasonic structural noise based on the microstructural properties of metals in the context of non destructive evaluation," in 13th International Symposium on Nondestructive Characterization of Materials (NDCM-XIII), 2013, pp. 20-24.

Dreyer, T., Krauss, W., Bauer, E., and Riedlinger, R., "Investigations of compact self focusing transducers using stacked piezoelectric elements for strong sound pulses in therapy," in 2000 IEEE Ultrasonics Symposium. Proceedings. An International Symposium (Cat. No. 00CH37121), vol. 2. IEEE, 2000, pp. 1239-1242.

Gustafsson, M. G., "Nonlinear clutter suppression using split spectrum processing and optimal detection," IEEE transactions on ultrasonics, ferroelectrics, and frequency control, vol. 43, pp. 109-124, 1996.

Gustafsson, M. G. and Stepinski, T., "Studies of split spectrum processing, optimal detection, and maximum likelihood amplitude estimation using a simple clutter model," Ultrasonics, vol. 35, pp. 31-52, 1997.

He, H., Li, J., and Stoica, P., Waveform design for active sensing systems: a computational approach. Cambridge University Press, 2012.

Hunter, D. R. and Lange, K., "A tutorial on mm algorithms," The American Statistician, vol. 58, pp. 30-37, 2004.

Jacobs, I. M. and Wozencraft, J., "Principles of communication engineering." 1965.

Kay, S., Intuitive probability and random processes using MATLABß. Springer Science \& Business Media, 2006.

Kay, S., "Optimal signal design for detection of gaussian point targets in stationary gaussian clutter/reverberation," IEEE journal of selected topics in signal processing 1.1, vol. 31, pp. 31-41, 2007.

Kay, S. M., Fundamentals of Statistical Signal Processing, Volume I: Estimation Theory. Prentice Hall PTR, 1993.

Kay, S. M., Fundamentals of Statistical Signal Processing: Detection. Prentice Hall PTR, 1998. 
Krautkrämer, J. and Krautkrämer, H., "Ultrasonic testing by determination of material properties," in Ultrasonic Testing of Materials. Springer, 1990.

Langenberg, K.-J., Marklein, R., and Mayer, K., Ultrasonic nondestructive testing of materials: theoretical foundations. CRC Press, 2012.

Laureti, S., Sfarra, S., Malekmohammadi, H., Burrascano, P., Hutchins, D. A., Senni, L., Silipigni, G., Maldague, X., and Ricci, M., "The use of pulsecompression thermography for detecting defects in paintings," $N d t \& 6$ E International, vol. 98, pp. 147-154, 2018.

Levanon, N. and Mozeson, E., Radar signals. John Wiley \& Sons, 2004.

Misaridis, A., "Ultrasound imaging using coded signals," 2001.

Nadarajah, S. and Kotz, S., "Exact distribution of the max/min of two gaussian random variables," IEEE Transactions on very large scale integration (VLSI) systems, vol. 16, no. 2, pp. 210-212, 2008.

Niederdränk, T., "Maximum length sequences in non-destructive material testing: application of piezoelectric transducers and effects of time variances," Ultrasonics, vol. 35, pp. 195-203, 1997.

Pillai, S. U., Youla, D., Oh, H., and Guerci, J. R., "Optimum transmit-receiver design in the presence of signal-dependent interference and channel noise," in Conference Record of the Thirty-Third Asilomar Conference on Signals, Systems, and Computers (Cat. No. CH37020), vol. 2. IEEE, 1999, pp. 870875 .

Pollakowski, M. and Ermert, H., "Chirp signal matching and signal power optimization in pulse-echo mode ultrasonic nondestructive testing," IEEE transactions on ultrasonics, ferroelectrics, and frequency control, vol. 41, pp. 655-659, 1994.

Price, R., "Chebyshev low pulse compression sidelobes via a nonlinear fm," in National Radio Science Meeting of URSI, 1979.

Quatieri, T. F., Discrete-time speech signal processing: principles and practice. Pearson Education India, 2006.

Ramuhalli, P., Cinson, A. D., Crawford, S. L., Harris, R. V., Diaz, A. A., and Anderson, M. T., "Evaluation of signal processing tools for improving phased array ultrasonic weld inspection," in Smart Sensor Phenomena, Technology, Networks, and Systems 2011, vol. 7982. International Society for Optics and Photonics, 2011. 
Ramuhalli, P., Good, M. S., Diaz, A. A., Anderson, M. T., Watson, B. E., Peters, T. J., Dixit, M., and Bond, L. J., "Ultrasonic characterization of cast austenitic stainless steel microstructure: Discrimination between equiaxedand columnar-grain material-an interim study," Pacific Northwest National Lab.(PNNL), Richland, WA (United States), Tech. Rep., 2009.

Rawashdeh, Y. and Kay, S., "A new physically motivated clutter model with applications to non-destructive ultrasonic testing," IEEE Transactions on Ultrasonics, Ferroelectrics, and Frequency Control, 2020.

Ricci, M., Callegari, S., Caporale, S., Monticelli, M., Battaglini, L., Eroli, M., Senni, L., Rovatti, R., Setti, G., and Burrascano, P., "Exploiting nonlinear chirp and sparse deconvolution to enhance the performance of pulsecompression ultrasonic ndt," in 2012 IEEE International Ultrasonics Symposium. IEEE, 2012, pp. 1489-1492.

Saniie, J., Wang, T., and Bilgutay, N. M., "Statistical evaluation of backscattered ultrasonic grain signals," The Journal of the Acoustical Society of America, vol. 84, pp. 400-408, 1988.

Skolnik, M. I., RADAR systems. McGraw-Hill, NY, 2001.

Song, J., Babu, P., and Palomar, D. P., "Optimization methods for designing sequences with low autocorrelation sidelobes," IEEE Transactions on Signal Processing, vol. 63, pp. 3998-4009, 2015.

Stoica, P., He, H., and Li, J., "New algorithms for designing unimodular sequences with good correlation properties," IEEE Transactions on Signal Processing, vol. 57, no. 4, pp. 1415-1425, 2009.

Stoica, P., Moses, R. L., et al., "Spectral analysis of signals," 2005.

Stoica, P. and Selen, Y., "Cyclic minimizers, majorization techniques, and the expectation-maximization algorithm: a refresher," IEEE Signal Processing Magazine, vol. 21, no. 1, pp. 112-114, 2004.

Thompson, J. and Titlebaum, E., "The design of optimal radar waveforms for clutter rejection using the maximum principle." 1967.

Van Trees, H. L. and Bell, K. L., Detection estimation and modulation theory. John Wiley \& Sons, 2004.

Wagner, R. F., "Statistics of speckle in ultrasound b-scans," IEEE Trans. Sonics $\&$ Ultrason., vol. 30, pp. 156-163, 1983.

Yalda-Mooshabad, I., Margetan, F. J., and Thompson, R. B., "Monte-carlo simulation of ultrasonic grain noise," in Review of progress in Quantitative Nondestructive Evaluation. Springer, 1993, pp. 1727-1734. 
Zhang, N. and Golomb, S. W., "Polyphase sequence with low autocorrelations," IEEE Transactions on Information Theory, vol. 39, pp. 1085-1089, 1993. 